OUTP-15-17P

SMU-HEP-15-12

TIF-UNIMI-2015-14

LCTS/2015-27

CERN-PH-TH-2015-249

\title{
PDF4LHC recommendations for LHC Run II
}

\author{
Jon Butterworth ${ }^{1}$, Stefano Carrazza ${ }^{2,4}$, Amanda Cooper-Sarkar ${ }^{3}$, Albert De Roeck ${ }^{4,5}$, Joël \\ Feltesse $^{6}$, Stefano Forte ${ }^{2}$, Jun Gao ${ }^{7}$, Sasha Glazov ${ }^{8}$, Joey Huston ${ }^{9}$, Zahari Kassabov ${ }^{2}, 10$, \\ Ronan McNulty ${ }^{11}$, Andreas Morsch ${ }^{4}$, Pavel Nadolsky ${ }^{12}$, Voica Radescu ${ }^{13}$, Juan Rojo ${ }^{14}$ and \\ Robert Thorne ${ }^{1}$. \\ ${ }^{1}$ Department of Physics and Astronomy, University College London, \\ Gower Street, London WC1E 6BT, UK. \\ 2 TIF Lab, Dipartimento di Fisica, Università di Milano and INFN, Sezione di Milano, \\ Via Celoria 16, I-20133 Milano, Italy \\ ${ }^{3}$ Particle Physics, Department of Physics, University of Oxford, \\ 1 Keble Road, Oxford OX1 3NP, UK. \\ ${ }^{4}$ PH Department, CERN, CH-1211 Geneva 23, Switzerland \\ ${ }^{5}$ Antwerp University, B2610 Wilrijk, Belgium \\ ${ }^{6}$ CEA, DSM/IRFU, CE-Saclay, Gif-sur-Yvette, France \\ ${ }^{7}$ High Energy Physics Division, Argonne National Laboratory, \\ Argonne, Illinois 60439, U.S.A. \\ 8 Deutsches Elektronen-Synchrotron (DESY), \\ Notkestrasse 85, D-22607 Hamburg, Germany. \\ ${ }^{9}$ Department of Physics and Astronomy, Michigan State University, \\ East Lansing, MI 48824 U.S.A. \\ 10 Dipartimento di Fisica, Università di Torino and INFN, Sezione di Torino, \\ Via Pietro Giuria 1, I-10125 Torino, Italy \\ ${ }^{11}$ School of Physics, University College Dublin Science Centre North, \\ UCD Belfeld, Dublin 4, Ireland \\ 12 Department of Physics, Southern Methodist University, Dallas, TX 75275-0181, U.S.A. \\ 13 Physikalisches Institut, Universität Heidelberg, Heidelberg, Germany. \\ ${ }^{14}$ Rudolf Peierls Centre for Theoretical Physics, 1 Keble Road, \\ University of Oxford, OX1 3NP Oxford, UK
}

\begin{abstract}
:
We provide an updated recommendation for the usage of sets of parton distribution functions (PDFs) and the assessment of $\mathrm{PDF}$ and $\mathrm{PDF}+\alpha_{s}$ uncertainties suitable for applications at the LHC Run II. We review developments since the previous PDF4LHC recommendation, and discuss and compare the new generation of PDFs, which include substantial information from experimental data from the Run I of the LHC. We then propose a new prescription for the combination of a suitable subset of the available PDF sets, which is presented in terms of a single combined PDF set. We finally discuss tools which allow for the delivery of this combined set in terms of optimized sets of Hessian eigenvectors or Monte Carlo replicas, and their usage, and provide some examples of their application to LHC phenomenology.

This paper is dedicated to the memory of Guido Altarelli (1941-2015), whose seminal work made possible the quantitative study of parton distribution functions.
\end{abstract}




\section{Contents}

1 Introduction 3

1.1 Parton distributions at the $\mathrm{LHC} \ldots \ldots \ldots \ldots \ldots$

1.2 The PDF4LHC Working Group and the 2010 recommendations . . . . . . . . . 3

1.3 Intermediate updates . . . . . . . . . . . . . . . 4

1.4 Scope of this document ...................... 5

2 Recent developments in PDF determination $\quad 7$

2.1 Intermediate PDF updates . . . . . . . . . . . . . . . 7

2.2 New experimental measurements . . . . . . . . . . . . . . 8

2.3 Current PDF sets and methodological improvements . . . . . . . . . . . 9 9

2.4 Origin of the differences between PDFs . . . . . . . . . . . . . . 12

2.4.1 Dependence on the heavy-quark scheme . . . . . . . . . . . . 13

2.4.2 Differences within the global PDF fits . . . . . . . . . . . . . 14

2.4.3 Differences in the size of the PDF uncertainties . . . . . . . . 15

3 Comparisons of PDF sets $\mathbf{1 6}$

3.1 Parton distributions . . . . . . . . . . . . . . . . . . 16

3.2 PDF luminosities . . . . . . . . . . . . . . . . . . . . 17

4 Constructing the PDF4LHC15 combination 19

4.1 Usage of PDF sets and their combinations . . . . . . . . . . . . . . 20

4.2 Statistical combination of PDF sets . . . . . . . . . . . . . 22

5 Implementation and delivery of the PDF4LHC15 PDFs 22

5.1 The Monte Carlo combination of PDF sets . . . . . . . . . . . . 24

5.2 The Monte Carlo reduction method: CMC-PDFs . . . . . . . . . . . 27

5.3 Hessian reduction methods . . . . . . . . . . . . . . . . . . 29

5.3 .1 META-PDFs . . . . . . . . . . . . . . . . 30

5.3 .2 MCH-PDFs ............................ 32

5.3 .3 Choice of Hessian sets . . . . . . . . . . . . . . 34

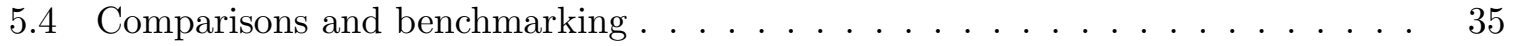

5.5 Implications for LHC processes . . . . . . . . . . . . . . . . . . 37

6 The PDF4LHC 2015 recommendations 46

6.1 Delivery and guidelines . . . . . . . . . . . . . . . . 47

6.2 Formulae for the calculation of $\mathrm{PDF}$ and $\mathrm{PDF}+\alpha_{s}$ uncertainties . . . . . . . . 49

6.3 PDF4LHC15 combined sets in the $n_{f}=4$ scheme . . . . . . . . . 51

6.4 Citation policy for the PDF4LHC recommendation . . . . . . . . . 52

7 Future directions 


\section{Introduction}

In this first section we introduce the general context for the updated PDF4LHC 2015 recommendations, and describe the layout of this document. Users whose main interest is the application of the PDF4LHC15 recommendations to their specific analysis can move directly to Sect. 6.

\subsection{Parton distributions at the LHC}

The accurate determination of the parton distribution functions (PDFs) of the proton is crucial for precision predictions at the Large Hadron Collider (LHC) 1 3. Almost all cross-sections of interest are now available at next-to-leading order (NLO), a rapidly increasing number also at next-to-next-to-leading order (NNLO), and even one, inclusive Higgs production in gluon fusion, at NNNLO [4]. The resulting improvements in theoretical uncertainties from the inclusion of higher order matrix elements demand a correspondingly improved control of PDF uncertainties.

A number of groups have recently produced updates of their PDFs fits [5 11] that have been compared to LHC data. Even for PDFs based on similar data sets, there are still variations in both the ensuing central values and uncertainties. This suggests that use of the PDFs from one group alone might underestimate the true uncertainties, and a combination of individual PDF sets is required for a robust uncertainty estimate in LHC cross-sections.

\subsection{The PDF4LHC Working Group and the 2010 recommendations}

The PDF4LHC Working Group has been tasked with:

1. performing benchmark studies of PDFs and of predictions at the LHC, and

2. making recommendations for a standard method of estimating $\mathrm{PDF}$ and $\mathrm{PDF}+\alpha_{s}\left(m_{Z}^{2}\right)$ uncertainties at the LHC through a combination of the results from different individual groups.

This mandate has led to several benchmarking papers 12, 13 and to the 2010 PDF4LHC recommendation [14] which has undergone several intermediate updates, with the last version available (along with a summary of PDF4LHC activities) from the PDF4LHC Working Group website:

http://www.hep.ucl.ac.uk/pdf4lhc/.

In 2010 the PDF4LHC Working Group carried out an exercise to which all PDF groups were invited to participate 12. Benchmark comparisons were made at NLO for LHC cross-section predictions at $7 \mathrm{TeV}$ using MCFM [15] as a common framework using carefully prescribed input files. The benchmark processes included $W / Z$ total cross-sections and rapidity distributions, $t \bar{t}$ cross-sections and Higgs boson production through $g g$ fusion for masses of 120, 180 and 240 $\mathrm{GeV}$. The PDFs used in this comparison included ABKM/ABM09 [16], CTEQ6.6/CT10 17, 18], GJR08 [19,20], HERAPDF1.0 [21], MSTW2008 [22] and NNPDF2.0 23]. The results were summarized in a PDF4LHC report [12] and in the LHC Higgs Cross Section Working Group Yellow Reports 1 [24] and 2 25]. In this study, each group used their native value of $\alpha_{s}\left(m_{Z}^{2}\right)$ along with its corresponding uncertainty. 
This 2010 PDF4LHC prescription [14] for the estimation of combined PDF $+\alpha_{s}\left(m_{Z}^{2}\right)$ uncertainties was based on PDFs from the three fitting groups performing a global analysis with a variable flavor number scheme, namely CTEQ, MSTW and NNPDF. Here, "global analysis" is meant to signify that the widest available set of data from a variety of experiments and processes was used, including deep-inelastic scattering, gauge boson Drell-Yan production, and inclusive jet production at hadron colliders.

The recommendation at NLO was to use the envelope provided by the central values and $\mathrm{PDF}+\alpha_{s}\left(m_{Z}^{2}\right)$ errors from CTEQ6.6, MSTW2008 and NNPDF2.0, using each group's prescription for combining the PDF with the $\alpha_{s}$ uncertainties 26 28, and with the central value given by the midpoint of the envelope. By definition, the extent of the envelope is determined by the extreme PDFs at the upper and lower edges. A drawback of this procedure was that it assigned a higher weight to these outlier PDF sets than what would be statistically correct when all the individual input sets have equal prior likelihood.

In addition, as each PDF uses its own native value of $\alpha_{s}\left(m_{Z}^{2}\right)$ and its own $\mathrm{PDF}+\alpha_{s}\left(m_{Z}^{2}\right)$ uncertainties about that central value, the resultant envelope inflates the impact of the $\alpha_{s}\left(m_{Z}^{2}\right)$ uncertainty. This very conservative prescription was adopted partly because of ill-understood disagreements between the PDF sets entering the combination (with other sets differing even more), and it was considered suitable specifically for the search of the Higgs boson at the LHC. Furthermore, it was suggested that in case of updates of the various PDFs set by the respective groups, the most recent sets should always be used.

At the time of this first recommendation, only MSTW2008 (of the three global fitting groups) had produced PDFs at NNLO. Since PDF errors are determined primarily by the experimental errors in the data sets, as well as by the methodology used in the corresponding PDF extraction (such as tolerance), it was known that PDF uncertainties were relatively unchanged when going from NLO to NNLO. The NNLO recommendation was thus to use MSTW2008 NNLO as the central result, and to take the same percentage error on that NNLO prediction as was found at NLO using the uncertainty prescription described above.

\subsection{Intermediate updates}

A follow-up benchmarking study in 2012 was carried out with the NNLO versions of the most up-to-date PDF sets [13]. By that time, CTEQ6.6 had been replaced by CT10 18,29 and NNPDF2.0 by NNPDF2.3 [30], both at NNLO. In addition, HERAPDF1.0 had been replaced by HERAPDF1.5 [31], and ABM09 by ABM11 [32]. In that study, a common value of $\alpha_{s}\left(m_{Z}^{2}\right)=0.118$ was used by all PDFs, with a variation between 0.117 and 0.119 to account for the uncertainty on $\alpha_{s}\left(m_{Z}^{2}\right)$.

Data from the LHC Run I was then already available, and detailed comparisons were made to data for inclusive jet and electroweak boson production from ATLAS [33, 34], CMS [35] and $\mathrm{LHCb}$ 36]. Much better agreement was observed between the updated versions of the three PDF sets used in the first study, CT10, MSTW2008, and NNPDF2.3, for example for the quark-antiquark PDF luminosity in the mass region of the $W / Z$ bosons, see Fig. 1. However some disagreements remained: Fig. 1 1 also shows the gluon-gluon PDF luminosity for $M_{X}$ values in the region of the Higgs boson mass. In this case, the resulting envelope was more than twice the size of the uncertainty band for any of the individual PDFs.

It is interesting to observe that the uncertainty bands for CT10, MSTW2008 and NNPDF2.3 are all reasonably similar, as shown in Fig. 2, even though the methodologies used to determine 

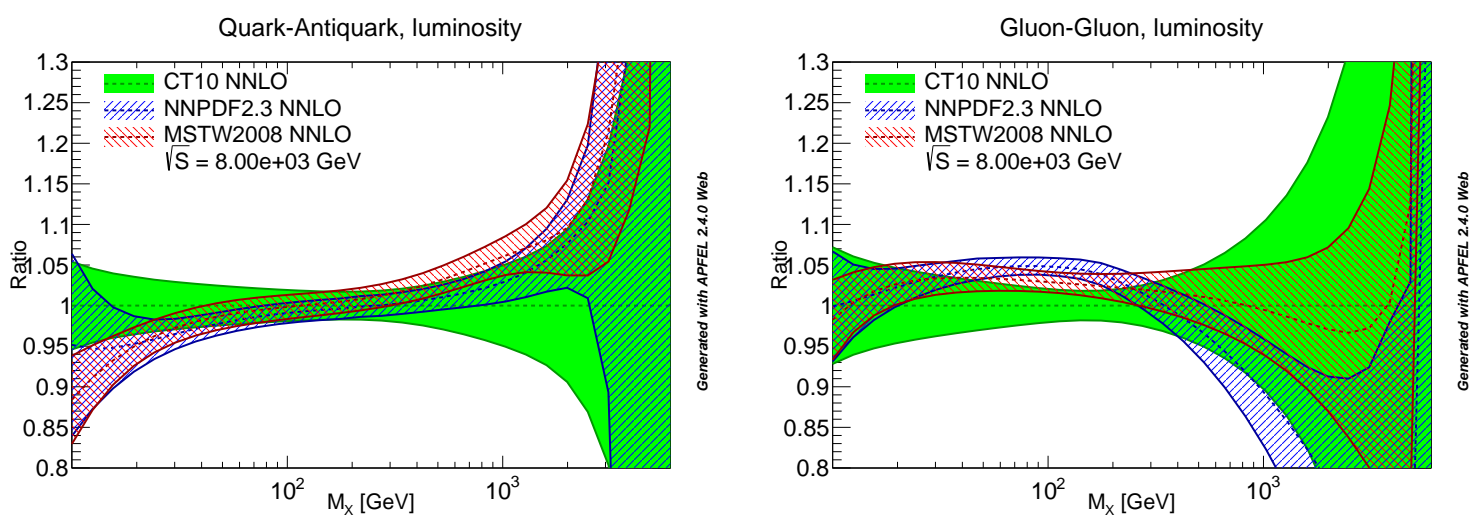

Figure 1: Comparison of the $q \bar{q}$ (left) and $g g$ (right) PDF luminosities at the LHC $8 \mathrm{TeV}$ for CT10, MSTW2008 and NNPDF2.3. Results are shown normalized to the central value of CT10.

them differ. The uncertainties for ABM11 are smaller, despite using a more limited data set, and also including sources of uncertainties due to $\alpha_{s}$ and heavy-quark masses; we will come back to this issue in Sect. 2.3 below. The uncertainties for HERAPDF1.5 were substantially larger, as expected from the more limited data set used.

Based on the 2012 study, the 2010 PDF4LHC recommendation was updated in 2013. First, it was recommended that the most up-to-date versions of the PDF sets from the three groups included in the previous recommendation be used, namely CT10, MSTW2008 and NNPDF2.3. Furthermore, as all sets now had both NLO and NNLO sets, it was recommended that the same procedure should be used both at NLO and NNLO. Finally, a somewhat simpler way of combining the PDF and $\alpha_{s}$ uncertainties was suggested. Namely, the central value of $\alpha_{s}\left(m_{Z}^{2}\right)$ was fixed for all PDFs to be $\alpha_{s}\left(m_{Z}^{2}\right)=0.118$, obtained by rounding off the then-current PDG world average $0.1184 \pm 0.0007$ [37] (and near the preferred value of each group anyway). An uncertainty range for $\alpha_{s}\left(m_{Z}^{2}\right)$ was taken to be \pm 0.002 at the $90 \%$ Confidence Level (CL) around the central value of 0.118 . This corresponds to a $68 \%$ CL uncertainty of \pm 0.0012 , somewhat more conservative than the PDG estimate.

The total $\mathrm{PDF}+\alpha_{s}\left(m_{Z}^{2}\right)$ uncertainty was then determined for each group by adding in quadrature the PDF uncertainty and $\alpha_{s}$ uncertainty, with the latter determined as the difference in the results found using the best-fit PDFs for the upper and lower $\alpha_{s}$ values of the $68 \%$ CL range. Indeed, it can be shown 27 that addition of PDF and $\alpha_{s}$ uncertainties in quadrature automatically accounts for the correlation between $\alpha_{s}$ and PDF uncertainties, assuming gaussianity and linear error propagation. As in the 2010 recommendation, it was then suggested to determine the $\mathrm{PDF}+\alpha_{s}$ uncertainty for each of the three groups, at the upper and lower $\alpha_{s}$ value of the chosen range $\alpha_{s}\left(m_{Z}^{2}\right)=0.118 \pm 0.0012$, and finally take the envelope of the results.

\subsection{Scope of this document}

The scope of the current document is to provide an update of the PDF4LHC recommendation suitable for its use at the LHC Run II. Similar to previous recommendations, the new recommendation will be based on a discussion and comparison of existing PDF sets. Specifically, we will argue that, based on more recent results, an envelope procedure is no longer necessary 

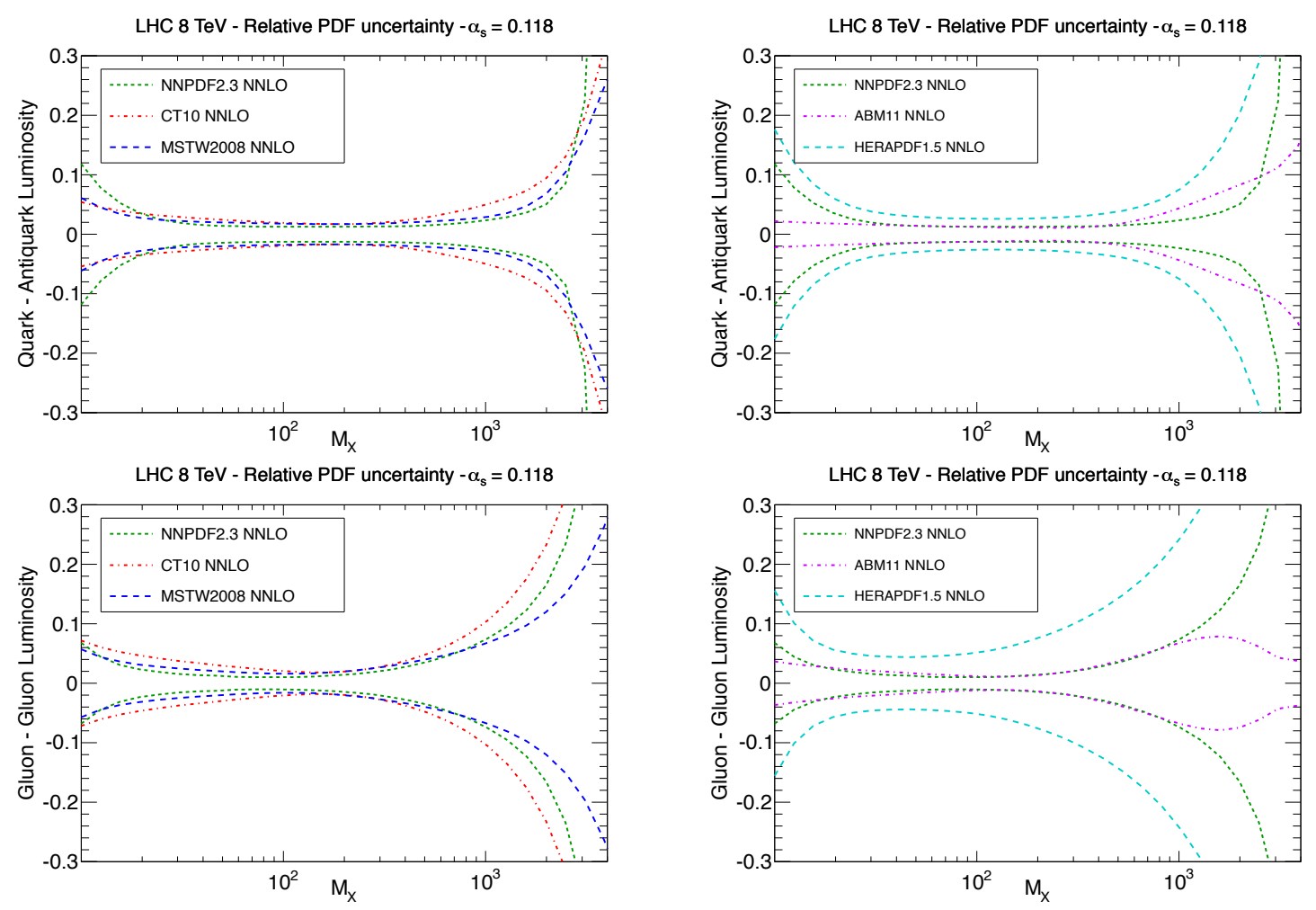

Figure 2: A comparison of PDF uncertainties in the $q \bar{q}$ (upper plots) and $g g$ luminosity (lower plots) at the LHC $8 \mathrm{TeV}$ for the ABM11, CT10, HERAPDF1.5, MSTW2008 and NNPDF2.3 NNLO PDFs, for a common value of the strong coupling $\alpha_{s}\left(m_{Z}^{2}\right)=0.118$. PDF uncertainties correspond to $68 \% \mathrm{CL}$ intervals.

and a purely statistical combination is appropriate. In addition, we will specify criteria for the inclusion of PDF sets in this combination. We will then discuss dedicated tools which allow for a streamlined construction and delivery of this statistical combination, and finally discuss the main feature of a combined PDF4LHC PDF set constructed in this way.

The outline of the paper is as follows. In Sect. 2 we review developments in PDF determination since the original PDF4LHC recommendation, and in Sect. 3 we compare the most recent PDF sets from all groups. In Sect. 4 we motivate the new prescription for the combination of PDF sets and enumerate criteria for the inclusion of PDF sets in this combination. In Sect. 5 we discuss the practical implementation of the 2015 PDF4LHC prescription, based on the combination of the CT14, MMHT14 and NNPDF3.0 PDF sets. First we introduce the Monte Carlo (MC) statistical combination, and we then present various methods suitable for the production and delivery of a manageable reduced combined PDF set, based on either MC or Hessian methodologies. We also present the resulting PDF4LHC15 combined sets, both at the level of individual PDFs and parton luminosities, and use them to compute several representative LHC cross-sections, specifically comparing the various delivery forms.

For the majority of the users of the new PDF4LHC15 recommendations, the only section which is essential is Sect. 6. This section presents a self-contained summary with the general guidelines for the usage of the PDF4LHC15 combined sets, including the formulae for the computations of $\mathrm{PDF}$ and $\mathrm{PDF}+\alpha_{s}$ uncertainties, and the corresponding citation policy. Finally, 
conclusions are drawn and directions for future developments are presented in Sect. 7.

\section{Recent developments in PDF determination}

Since the 2010 PDF4LHC recommendation document 14] there has been very considerable progress in the determination of PDFs. This includes both the usage of an increasingly large and diverse set of data, exploiting new measurements from the LHC, Tevatron and HERA experiments, and various methodological developments, which have been incorporated in various new PDF sets released by different groups. In this section we will give a brief summary of these developments.

\subsection{Intermediate PDF updates}

Some of the updates in PDF sets were fully expected at the time of the last recommendation document, and, as mentioned in Sect. 1. have been already implemented in updated recommendations, available at the PDF4LHC website 38 .

Specifically, at the time of the previous recommendation the most updated set from NNPDF was NNPDF2.0 [23], obtained using the zero-mass variable-flavour-number (ZM-VFN) scheme, which is known to miss important mass-dependent terms for scales near the quark mass and to induce inaccuracies in the PDFs. However, soon after the recommendation this set was updated to NNPDF2.1 [39] which used the FONLL general-mass variable flavour number scheme (GM-VFN scheme) described in [40], and this automatically improved general agreement with CT and MSTW PDFs. These PDFs were later also made available at NNLO [41]. Subsequently NNPDF updated their PDFs to the NNPDF2.3 set [30], the main change being the inclusion of early LHC data on rapidity-dependent vector boson production and asymmetries and on inclusive jet production. In addition, the theoretical treatment of charged-current structure functions in neutrino DIS was improved in NNPDF2.3, leading to a somewhat enhanced strangeness around $x \simeq 10^{-2}$. Even though changes from NNPDF2.1 to NNPDF2.3 are moderate, the new data and improved fitting methodology reduced uncertainties a little.

In 2013 the CT10 NNLO PDFs were released, specifically using the extension to NNLO [42] of the S-ACOT- $\chi$ heavy flavour scheme used by this group. This enabled an intermediate improved prescription based on the envelope of CT10, MSTW08 and NNPDF2.3 at NLO and NNLO, as discussed in Sect. 1 .

Coming to MSTW08, it was soon clear that despite good predictions for most LHC observables, the MSTW2008 PDFs did not describe low rapidity lepton asymmetry data, which is sensitive to small- $x(x \sim 0.01)$ valence quarks. This was rectified in an intermediate update, the MMSTWW (or sometimes MSTWCPdeut) PDFs [43], based on the same data sets as MSTW2008, but with an extended PDF parametrization based on Chebyshev polynomials in $(1-2 \sqrt{x})$ rather than just powers of $\sqrt{x}$, and more flexible deuteron correction (given as a function with four free parameters that are fit to data). This included a study of the number of terms in the parametrization of quarks and gluons required to reproduce functions to an accuracy of small fractions of a percent: seven parameters were found to be sufficient. These PDFs agree well with LHC $W$ asymmetry data, even though the data itself is not actually included in the fit.

There have also been intermediate updates from groups not included in the 2010 PDF4LHC combination. PDF sets from the HERAPDF group are based on fitting to HERA data only. 
While the HERA structure function data is undoubtedly the most constraining single data set available, particularly for the gluon and the total quark singlet distribution at moderately and small $x$, it provides fewer constraints in some kinematic regions (such as the gluon at large $x$ ) and some PDF combinations, and specifically it does not allow a separation of the down and strange distribution. At the time of the last recommendation, the most recent available PDF set was HERAPDF1.0, which was obtained from a fit to the HERA Run-I combined total cross-section data. This set was in good broad agreement with CT, MSTW and NNPDF sets, but with larger uncertainties for many PDF regions and a much softer high$x$ gluon and harder high- $x$ sea quarks. Since then, there have been a number of updates. HERAPDF1.5 [31] included some preliminary data on inclusive cross sections from HERA-II running. This reduced uncertainties slightly, and also led to a harder high- $x$ gluon and seaquark distributions at NNLO, though not as much at NLO. These differences between NLO and NNLO can be partly explained by the fact that the NNLO sets appeared some time after the NLO ones, and also that the NNLO HERAPDF1.5 set was based on a more flexible PDF parametrisation that its NLO counterpart.

There are also two other PDF sets that are based on smaller total sets of data than the CT, MMHT and NNPDF fits. These PDFs differ from all those discussed so far in that they adopt a fixed-flavour-number (FFN) scheme for the treatment of heavy quarks: i.e., they do not introduce PDFs for charm and bottom quarks and antiquarks. The most recent sets from these groups at the time of the previous recommendation were ABKM09 [16] and (G)JR09 [19, 44]. The group responsible for the former, now the ABM collaboration, has since produced two updated sets. The first one was the ABM11 PDFs [32], which included the combined HERA Run-I cross-section data for the first time, and also some $\mathrm{H} 1$ data at different beam energies in addition to the fixed target DIS and Drell-Yan data already used in the ABKM analysis. The PDFs were mostly unchanged, but with a slightly larger small- $x$ gluon. As in the ABKM analysis a rather low value of $\alpha_{s}\left(m_{Z}^{2}\right)$, i.e. $\alpha_{s}\left(m_{Z}^{2}\right)=0.1134$ at NNLO, was obtained.

In Ref. [45], the impact on the ABM11 analysis of fitting different jet datasets from D0, CDF and the 2010 ATLAS data was studied. This was done both at NLO and at NLO* (including the effects of threshold resummation) in jet production. As compared to the default fit, once the Tevatron jet data sets were included, the fitted value of $\alpha_{s}\left(m_{Z}^{2}\right)$ was found to vary by an amount ${ }_{-0.002}^{+0.001}$, depending on the particular data set and jet algorithm. These jet measurements have not been included in the posterior ABM PDF releases.

All these PDF fitting groups have presented major updates recently: MMHT14 [10], CT14 6] NNPDF3.0 [11], ABM12 [5] and HERAPDF2.0 [9], which will be discussed in more detail in Sect. 2.3 below.

\subsection{New experimental measurements}

One obvious reason for updates of the various PDF sets is the availability of new measurements from a variety of collider and fixed-target experiments. Recently, an up-to-date overview of all PDF-sensitive measurements at LHC Run-I has been presented in the "PDF4LHC report on PDFs and LHC data" 1. We refer to this document for an extensive discussion (including full references) and here we only provide a very brief summary.

First of all, HERA has provided combined H1 and ZEUS data on the charm structure functions, in addition to the already available combined inclusive Run-I structure function data. Run-II ZEUS and H1 data have also been published, and a legacy HERA combination of 
all inclusive structure function measurements from Run-I and Run-II has been presented very recently [9]; it is only included in the HERAPDF2.0 set. Also, Tevatron collaborations are still releasing data on lepton asymmetry and top-pair cross-sections and differential distributions.

A wide variety of data sets which help constrain the parton distributions has been made available at the LHC from the ATLAS, CMS and LHCb collaborations. This includes data sets on rapidity- and mass-dependent $W^{ \pm}, Z$ and $\gamma^{\star}$ production, inclusive jet cross-sections, inclusive top-pair production and $W+$ charm quark production, differential top pair production and dijet cross sections.

Many of these measurements have been included in recent global PDF sets. Therefore, for all PDF groups the fitted dataset is now considerably wider than that at the time of the 2010 PDF4LHC recommendation.

\subsection{Current PDF sets and methodological improvements}

We now discuss in turn all the presently available PDF sets. These differ from previous releases not only because of the inclusion of new data, but also because of various methodological and theoretical improvements, which we also review.

We first consider the most updated PDF sets from the three groups represented in the original recommendation. An important observation is that the most recent sets from these groups, CT14, MMHT2014 and NNPDF3.0 sets are all made available with full PDF uncertainty information available at the common value of $\alpha_{s}\left(m_{Z}^{2}\right)=0.118$, both at NLO and NNLO. This is to be contrasted to the situation at the time of the previous recommendation, when full information of PDF uncertainties was only available in each set for fixed (different) values of $\alpha_{s}$, spanning a range of approximately $\Delta \alpha_{s}\left(m_{Z}^{2}\right) \approx 0.002$. We now discuss each set in turn.

The CT14 PDF sets [6] have been made recently available at NLO, NNLO, and also at LO. These sets include a variety of LHC data sets as well as the most recent D0 data on electron charge asymmetry [46] and combined H1+ZEUS data on charm production [47. The PDFs also use an updated parametrization based on Bernstein polynomials, which reduces parameter correlation. The PDF sets contain 28 pairs of eigenvectors. LHC inclusive jet data are included at NLO also in the NNLO fit, despite the lack of a full NNLO calculation, in the expectation that the NNLO corrections are relatively small for single-inclusive cross-sections. The main change in the PDFs as compared to CT10 is a softer high- $x$ gluon, a smaller strange quark (partially due to correction of the charged current DIS cross-section code) and the details of the flavour decomposition, e.g. $\bar{u} / \bar{d}$ and the high- $x$ valence quarks, due both to the parametrization choices and new data. In the CT14 publication [6] a number of additional comparisons were made to data not included in the fit, such as $W+$ charm and $\sigma_{\bar{t} t}$ from the LHC, and the predictions are found to be in good agreement. One should also mention that the CT14 PDF uncertainties are provided as $90 \%$ confidence level intervals, that need to be rescaled by a factor 1.642 to compare with other PDF sets, for which uncertainties are provided as $68 \%$ confidence level intervals.

We now consider PDFs from the MSTW group, currently renamed MMHT due to a change in personnel. The MMHT14 PDFs [10] incorporate the improved parametrization and deuteron corrections in the MMSTWW study [48], and also a change in the GM-VFN scheme to the "optimal" scheme in 49, and a change in the branching fraction $B_{\mu}=B(D \rightarrow \mu)$ used in the determination of the strange quark from $\nu N \rightarrow \mu \mu X$ data. Charged-current structure functions were also updated in the MMHT14 analysis, in particular by including the NLO 
gluon coefficient functions. The updated analysis includes new data: the combined HERAI inclusive structure function data [21] (which post-dates the MSTW2008 PDFs) and charm structure function data, updated Tevatron lepton asymmetry data, vector boson and inclusive jet data from the LHC (though LHC jet data is not included at NNLO), and top pair crosssection data from the Tevatron and LHC. No PDFs change dramatically in comparison to MSTW2008, with the most significant changes being the shift in the small- $x$ valence quarks already observed in the MMSTWW study, a slight increase in the central value of the strange quark to help the fit to LHC data, and a much expanded uncertainty on the strange distribution due to the inclusion of a conservative uncertainty on $B_{\mu}$. The MMHT14 PDFs provide a much better description to the LHC $W$ lepton asymmetry data 34,50 than its predecessor MSTW08, thanks to the various improvements already implemented in the MMSTWW (also known as MSTW08CPdeut) intermediate release. Deuteron nuclear corrections are fitted to the data, and their uncertainty propagates into the total PDF uncertainty.

The MMHT14 PDFs are made available with 25 eigenvector pairs for $\alpha_{s}\left(m_{Z}^{2}\right)=0.118$ and 0.120 at NLO and 0.118 at NNLO, as well as at LO (for $\left.\alpha_{s}\left(m_{Z}^{2}\right)=0.135\right)$. However, $\alpha_{s}\left(m_{Z}^{2}\right)$ is also determined by the NLO and NNLO fits and values of $\alpha_{s}\left(m_{Z}^{2}\right)=0.1201$ and 0.1172 respectively are found, or 0.1195 and 0.1178 if the world average of $\alpha_{s}\left(m_{Z}^{2}\right)$ is included as a data point. These are in good agreement with the PDG world average without DIS data of $\alpha_{s}\left(m_{Z}^{2}\right)=0.1187 \pm 0.0007[107]$. A dedicated study of the uncertainties in the determination of $\alpha_{s}\left(m_{Z}^{2}\right)$ in the MMHT14 analysis has been presented in 51 .

The NNPDF3.0 PDF sets [11] are the recent major update within the NNPDF framework. In comparison to NNPDF2.3, NNPDF3.0 includes HERA inclusive structure function Run-II data from $\mathrm{H} 1$ and ZEUS (before their combination), more recent ATLAS, CMS and LHCb data on gauge boson production and inclusive jets, and $W+$ charm and top quark pair production. A subset of jet data was included at NNLO using an approximate NNLO treatment, based on a study [52] of the region where the threshold approximation [53] is reliable by comparing with the exact $g g$ channel calculation [54]. Heavy quarks are treated using the FONLL-B scheme (FONLL-A was used in NNPDF2.1 and NNPDF2.3), which includes mass corrections to an extra order in $\alpha_{s}$, thereby achieving a better description of low- $Q^{2}$ data on the charm structure function. The compatibility of the NNPDF3.0 NLO set with the LHCb forward charm production cross-sections has been demonstrated in [55], where it was also shown that these data could be useful in reducing the uncertainty on the small- $x$ gluon.

The NNPDF3.0 fitting procedure has been tuned by means of a closure test, namely, by generating pseudo-data based on an assumed underlying set of PDFs. One verifies in this case that the output of the fitting procedure is consistent with the a priori known answer. As a byproduct, one can investigate directly the origin of PDF uncertainties, and specifically how much of it is due to the uncertainty in the data, how much it is due to the need to interpolate between data points, and how much is due to the fact that there is an infinite number of functions which produce equally good fits to a given finite number of data-points. The minimization has been optimized based on the closure test, specifically by choice of a more efficient genetic algorithm, and the choice of optimal stopping point based on crossvalidation and the search for the absolute minimum of the validation $\chi^{2}$ (look-back fitting). The NNPDF3.0 PDFs display moderate changes in comparison to NNPDF2.3: specifically somewhat smaller uncertainties and a noticeable change in the gluon-gluon luminosity.

The HERAPDF2.0 PDFs has also become recently available 9. HERAPDF2.0 is the only PDF set to include the full legacy Run-I and Run-II combined HERA structure function data, 
based on runs at beam energies of $920,820,575$ and $460 \mathrm{GeV}$. This PDF set has considerably reduced uncertainties on PDFs compared to HERAPDF1.0. In particular there is a much improved constraint on flavour decomposition at moderate and high $x$ due to the difference between neutral current $e^{+}$and $e^{-}$cross-sections, and due to much more precise charged current data, which provides a genuine constraint on the $d_{V}$ distribution. The running at different energies gives sensitivity to $F_{L}\left(x, Q^{2}\right)$ which provides some new information on the gluon. The HERAPDF2.0 sets are made available also with a default of $\alpha_{s}\left(m_{Z}^{2}\right)=0.118$ and with 14 eigenvector sets along with further variations to cover uncertainties due to model assumptions and changes in the form of the parametrization; for CT, MMHT and NNPDF this is not necessary because the input form of the PDFs is flexible enough. Sets including HERA jet and charm data have also been made available. The HERAPDF2.0 PDFs agree fairly well in general with the CT, MMHT and NNPDF sets, but there are some important differences in central values, particularly for high- $x$ quarks. Overall, the uncertainties on HERAPDF2.0 PDFs are markedly smaller than for previous versions, but there are still some regions where the PDFs have significantly larger uncertainties than those in the global fits.

Let us also mention that the three general-mass variable-flavour number schemes used by these four PDF sets (S-ACOT- $\chi$, FONLL and RT/RT ${ }^{\text {opt }}$ ) exhibit a remarkable convergence, especially at NNLO [56]. All these four sets fit the HERA charm cross-sections with comparable quality, and residual differences between the three GM-VFN schemes translate into rather small differences in the resulting PDFs. [57

The most recent update for the ABM collaboration, ABM12 [5], now includes the HERA combined charm cross-section data, an extension of the HERA inclusive data to higher $Q^{2}$ than previously used, and vector boson production data from ATLAS, CMS and LHCb. The heavy flavour contributions to structure function data are now calculated using the $\overline{M S}$ renormalization scheme [58] rather than the pole mass scheme. The main change compared to ABM11 PDFs is in the details of the decomposition into up and down quarks and antiquarks, this being affected by the LHC vector boson data. The ABM12 PDF sets are determined together with $\alpha_{s}$, whose value comes out to be rather lower than the PDG average, namely $\alpha_{s}\left(m_{Z}^{2}\right)=0.1132 \pm 0.0011$ at NNLO. Top quark pair production data from the LHC is investigated, but not included in the default PDFs. Its inclusion tend to raise the high- $x$ gluon and $\alpha_{s}\left(m_{Z}^{2}\right)$ a little; the precise details depend on the precise value of the top quark mass (and mass renormalization scheme) used. The ABM12 PDFs are available with 28 eigenvector pairs for the default $\alpha_{s}\left(m_{Z}^{2}\right)$ and at NNLO only. Within the same framework there has been a recent specific investigation of the strange sea [59] in the light of LHC data and NOMAD and CHORUS fixed-target data, but this is not accompanied by a PDF set release. More recently, an update of the ABM12 with additional data from gauge boson production at the Tevatron and the LHC has been presented [60], though this study is not accompanied by the release of a new PDF set.

There has also been an update of the (G)JR PDFs: JR14 8. The default sets from this group make the assumption that PDFs must be valence-like at low $Q_{0}^{2}=0.8 \mathrm{GeV}^{2}$. This analysis extends and updates the fixed-target and collider DIS data used, in particular including CCFR and $\mathrm{NuTeV}$ dimuon data as a constraint on strange quarks, and includes both HERA and Tevatron jet data at NLO, but does not include any LHC data. The strong coupling is also determined along with the PDF, yielding a value $\alpha_{s}\left(m_{Z}^{2}\right)=0.1136$ at NNLO, and a large small- $x$ gluon distribution. There are some significant changes compared to the JR09 NNLO PDFs. These are mainly at high $x$ values, where there is an increase in down and 
strange quarks and antiquarks, and a decrease in the gluon. At low $x$ values, the features are largely determined by the assumption of a valence-like input, and are largely unchanged. A determination without the valence-like assumption (and thus a more flexible parametrization) is also performed, leading to PDFs and a best fit value of $\alpha_{s}\left(m_{Z}^{2}\right)$ more in line with other groups, though it is not adopted as a default.

In addition, there has been a number of more specific PDF studies. The CJ12 PDFs 7 emphasize the description of the high- $x$ region, with particular care paid to higher-twist corrections and the modeling the deuteron corrections to nucleon PDFs, thereby allowing for the inclusion of high- $x$ and low $Q^{2}$ data which is often cut from other fits, most notably Jefferson Lab data. Several analyses have been focused on the possible intrinsic charm component of the proton. Specifically, Refs. 61,62 have presented investigations of the evidence for, and limits on, intrinsic charm in the proton. Work in progress towards the determination of intrinsic charm is also ongoing within NNPDF, and the required modifications of the FONLL GM-VFN scheme have been presented in 63. The determination of mass of the charm quark from PDF fits has been discussed in Refs. 64 65]. PDFs including QED corrections, which were presented for the first time in Ref. [66] by the MRST group, have been updated in Ref. [67]; a determination of the photon PDF from the data has been presented for the first time in [68], with the corresponding study in the CT14 framework found in Ref. [69].

It is also important to mention that a number of PDF-related studies based on the opensource HERAfitter framework 70 have also become available. From the HERAfitter developers team, two studies have been presented: PDFs with correlations between different perturbative orders [71] and the impact on PDFs of the Tevatron legacy Drell-Yan measurements [72]. Within the LHC experiments, various studies of the constraints of new measurements on PDFs have been performed using HERAfitter, including the ATLAS $W, Z$ analysis [73] that lead to a strangeness determination and the CMS studies constraining the gluon and $\alpha_{s}\left(m_{Z}^{2}\right)$ from inclusive jet production $74,75$. . The impact of the LHCb forward charm production data on the small- $x$ gluon has been explored by the PROSA collaboration in 76. Finally, HERAfitter has also been used to quantify the impact on PDFs of data from a future Large Hadron Electron Collider (LHeC) [77].

\subsection{Origin of the differences between PDFs}

There have been many developments in understanding the differences between the PDF sets obtained by different groups. This understanding inevitably leads to an improvement of the best procedures for combining these different PDFs. In the benchmark document [12], on which the first PDF4LHC recommendation was based, the differences between the PDFs and the consequent differences in predictions for LHC cross-sections were discussed; subsequent benchmarking exercises were published in Refs. [32,78]; and then in [13 including comparisons with published LHC measurements. See also 79 for an earlier benchmark exercise. Here we concentrate on three topics on which some progress has been made recently: the impact of the choice of heavy-quark scheme; the origin of the remaining differences between global PDF fits; and the origin of the differences between the sizes of PDF uncertainties. Graphical comparisons of the PDFs illustrating their present level of agreement are presented in Sect. 3 . 


\subsubsection{Dependence on the heavy-quark scheme}

The results of various benchmark studies [13,32,78] illustrate how the softer high- $x$ gluon and smaller $\alpha_{s}\left(m_{Z}^{2}\right)$ present in PDF sets like ABM12 and JR09 leads to a smaller prediction for top pair cross sections and Higgs bosons as compared to the global PDF sets. In both cases, the magnitude of the difference depends on whether the default $\alpha_{s}\left(m_{Z}^{2}\right)$ is obtained directly from the fit or if it is treated as an external input. In these sets also the quark PDFs seem to lead to slightly larger vector boson production cross-sections as compared to the global fits. On the other hand the CT, MSTW/MMHT, and NNPDF, and HERAPDF PDFs at NNLO are in generally good agreement, though HERAPDF has associated rather larger uncertainties from the reduced dataset.

In the previous recommendation it was stated that the systematic feature of a lower high- $x$ gluon and lower value of $\alpha_{s}\left(m_{Z}^{2}\right)$ in some fits was due to the omission of Tevatron jet data, which constrains the high- $x$ gluon. The understanding of this issue has improved. While it is true that Tevatron and LHC jet data directly constrain the gluon distribution, and that PDF sets with a particularly small high- $x$ gluon distribution and/or small value of $\alpha_{s}\left(m_{Z}^{2}\right)$ do not provide the best fit to these data (see [45, 80 for discussions on this issue with similar results, though not the same conclusions), it is unclear whether their omission automatically leads to a small high- $x$ gluon distribution or small $\alpha_{s}\left(m_{Z}^{2}\right)$. The results of including the jet data in the fit also show some dependence on the treatment of correlated systematic errors (additive or multiplicative) assumed, though these effects are way too small [11,29] to explain the aforementioned differences.

The behavior of the gluon is constrained directly by Tevatron/LHC jet production at high $x$, and indirectly by scaling violations in DIS data at very small and high $x$, with the latter influencing the gluon at moderate $x$ via the momentum sum rule. The gluon PDF is strongly correlated with $\alpha_{s}\left(m_{Z}^{2}\right)$ at high $x$ and anti-correlated at low $x$. Due to this feature of correlation between the gluon and $\alpha_{s}\left(m_{Z}^{2}\right)$, there is no automatic strong pull to lower $\alpha_{s}\left(m_{Z}^{2}\right)$ or small high- $x$ gluon in the fits that omit hadron collider data [80,81. It appears that the DIS and Drell-Yan data provide only a weak constraint on $\alpha_{s}\left(m_{Z}^{2}\right)$ and the gluon, if both are left free, a conclusion also evident in those HERAPDF1.6 studies that added HERA jet data to the DIS data and more strongly constrain $\alpha_{s}\left(m_{Z}^{2}\right)$.

An analysis in the ABM framework [82 suggested that this issue is related to whether one makes a conservative cut on low- $Q^{2}$ structure function data, or includes it and includes higher-twist corrections as well as higher-order QCD corrections to $F_{L}\left(x, Q^{2}\right)$ in fits of the NMC cross-section data. However, their findings were not corroborated by CTEQ, MSTW and NNPDF groups 80,83 85], who did not observe clear-cut sensitivity of the PDFs to the details of the NMC treatment either at fixed $\alpha_{s}\left(m_{Z}^{2}\right)\left[29,83\right.$, or varying $\alpha_{s}\left(m_{Z}^{2}\right)[29,80]$. Also, no significant variation in PDFs was seen when including a fixed higher twist in the NNPDF2.3 analysis in [84]. In [85, when the higher-twist contribution is fit within the standard MSTW framework, there was fairly small impact on the PDFs, and less on $\alpha_{s}\left(m_{Z}^{2}\right)$.

However, different schemes for heavy-quark treatment, notably the usage of the fixedflavor scheme as opposed to general-mass variable flavor number scheme, may explain the most pronounced disagreements observed in the gluon distribution between ABM/JR and the global sets. When a fit was performed to DIS data using the 3-flavor FFN scheme at NLO using the MSTW and NNPDF frameworks [49, 84], the gluon became softer at high $x$ and larger at small $x$; the light quarks became larger at small $x$ (a feature previously noted to some extent 
in 86$])$; the value of $\alpha_{s}\left(m_{Z}^{2}\right)$ decreased noticeably. This was confirmed in more detail in [85], using an approximate NNLO FFN scheme. At NNLO the decrease in $\alpha_{s}\left(m_{Z}^{2}\right)$ was more marked that at NLO, and these conclusions did not change after the Drell-Yan data and higher-twist corrections were added. It was also observed that the quality of the fit to the DIS data was clearly worse when using the FFN scheme, as opposed to the GM-VFN scheme. At a fixed $\alpha_{s}\left(m_{Z}^{2}\right)$ the same conclusions about the PDFs, more detailed confirmation of a worse fit were found, while it was also observed that the deterioration is mainly in high- $Q^{2}$ DIS data [84].

In 85 a theoretical investigation of the difference between the speed of evolution of $F_{2}^{c}\left(x, Q^{2}\right)$ at high $Q^{2}$ was performed, and it was shown that, for the region $x \sim 0.05$, there is slow convergence of the $\ln \left(Q^{2} / m_{c}^{2}\right)$ terms included at finite order in the FFNS to the fully resummed result in a GM-VFNS, leading to the worse fit to HERA data for this range of $x$ at high $Q^{2}$. It was also shown that this slower evolution is partially compensated by an increase of the gluon in this region of $x$, which feeds down to lower $x$ with evolution. This, however, also results in a smaller gluon at high $x$, so that the fit to the fixed-target data then requires a lower value of $\alpha_{s}\left(m_{Z}^{2}\right)$. This feedback between $\alpha_{s}\left(m_{Z}^{2}\right)$ and the gluon is somewhat enhanced if higher twist contributions are included, as the freedom in these provides more flexibility to change the PDFs and $\alpha_{s}\left(m_{Z}^{2}\right)$ without worsening the fit quality.

The general conclusion is thus that smaller values of $\alpha_{s}\left(m_{Z}^{2}\right)$ (particularly at NNLO), softer high- $x$ gluons, and slightly enhanced light quarks at high $x$ are found if a FFNS, rather than GM-VFNS, is adopted in the PDF fit. In an extremely conservative approach, these differences might be viewed as a "theoretical uncertainty", but the markedly worse fit quality for the FFNS motivates instead that a GM-VFNS should be adopted, especially for calculating quantities sensitive to high- $Q^{2}$ PDFs.

\subsubsection{Differences within the global PDF fits}

While the above discussion sheds light on the differences between the sets based on a FFN scheme and those which adopt a GM-VFN scheme, the fact remains that CT, HERAPDF, MSTW and NNPDF also show some smaller differences, even though they all adopt a GM-VFN scheme. As mentioned in the introduction, for the previous families of each group, NNPDF2.3, CT10 and MSTW08, these differences were especially noticeable for the gluon PDF and led to a spread of predictions for Higgs production via gluon fusion, producing an envelope uncertainty that is more than twice the size of individual PDF uncertainties.

An attempt to understand the origin of these moderate differences was made in Ref. [57]. In this study, all groups produced fits to the HERA combined Run-I inclusive cross-section data only. Furthermore, all structure function calculation codes from the groups were benchmarked using a toy set of PDFs. The agreement was good in general, with all deviations in neutral current processes being due only to the differing choices in GM-VFNS choice, which were much smaller at NNLO than NLO due to the convergence of such schemes, and differing treatments of electroweak corrections at high $Q^{2}$. A difference in charged current cross-sections was noted, and corrected, but it did not affect the PDFs extracted in any significant manner. Fits to the data were performed adopting a common choice of kinematic cuts, $\chi^{2}$ definition (i.e. treatment of correlated uncertainties) and charm mass, all of which differ to some extent in the default fits.

This study found that the PDFs and predictions for Higgs cross-sections at NNLO still followed the same pattern: in particular, $\sigma_{g g \rightarrow h}$ for CT remained smaller than the MSTW 
result, which in turn was smaller than the NNPDF prediction, as in the global fits, even though all predictions were compatible within the large uncertainties of a HERA-only fit. An investigation of variations of the heavy flavour scheme was also performed, but this turned out to have a comparatively small impact. Some evidence of parametrization dependence between CT and MSTW was noted (and new parametrization forms were adopted by CT), but it was not conclusive.

Concurrent to this study with DIS data, NLO theoretical calculations for fitting collider jet data, based on programs MEKS [87] and NLOJET++ 88,89], and their fast interfaces APPLgrid and FastNLO [90 93, were validated [13,94,95], providing greater confidence in the NLO theoretical codes and their fast interfaces, and the fits to the jet data.

Therefore, the details of the reasons for the disagreement at, or around, the one- $\sigma$ level, between the global fits for the gluon luminosity in the region of the Higgs boson mass were still only partially understood. This was a situation that conservatively could only be handled by taking an envelope of results, which amounts to saying that one or more of the results are affected by an unknown source of systematics (or bias).

This state of affairs has changed completely with the release of the most recent CT14, MMHT14 and NNPDF3.0 PDF sets. Indeed, due to increased data constraints and methodological improvements in each of the fitting procedures, these turn out to be in better agreement for most PDFs in a wide range of $x$, as will be illustrated in Sect. 3 .

\subsubsection{Differences in the size of the PDF uncertainties}

In general the sizes of the PDF uncertainties between the three global sets, NNPDF, CT and MMHT, turn out to be in rather reasonable agreement. On the other hand, the sizes of the uncertainties for PDF sets based on a HERA-only dataset, such as HERAPDF, are be expected to be larger, and in some cases much larger, than those of the global fits. This is indeed what happens for the HERAPDF2.0 PDFs, despite including the legacy HERA combination data [9], since deep-inelastic scattering cannot constrain a number of quark flavor combinations and the medium and large- $x$ gluon. The much larger PDF uncertainties in HERA-only fits as compared to global fits have also been verified in the NNPDF framework. The NNPDF3.0 HERA-only fit [11] indeed shows substantially larger uncertainties for most PDF combinations (with the exception of the quark singlet and the small- $x$ gluon) than the corresponding global analysis.

Whereas HERAPDF2.0 includes the legacy HERA combination data 9, which are not included in any other fit, it has been shown 96, 97, that the impact of these data on a global fit that already included the HERA-I data was moderate. In addition, it has been checked 97 that their impact on a global fit like NNPDF3.0 which already included both the HERA-I combination and the individual HERA-II measurements is very small. Therefore, the availability of the legacy HERA inclusive dataset does not modify the general anticipation that HERA-only fits (or more general, DIS-only fits) will have larger PDF errors than the global fits. However, it is worth emphasizing that the uncertainties of HERAPDF2.0 are in many cases comparable (for the $u$ quark) or even smaller (for the gluon) to the global fits, as will be illustrated in the comparisons of Sect. 3 ,

The PDF uncertainties in ABM12 are systematically smaller, and often much smaller, that those of the global fits. Therefore the PDF uncertainty estimates of the three global fits, on one hand, and those of ABM12, on the other, do not appear to be consistent. The most likely explanation for the disparate sizes of the uncertainties is their varied statistical 
interpretations adopted by the PDF analysis groups. In the global sets, they are determined according to complex procedures, and include more sources of uncertainties than the purely experimental uncertainty obtained from the $\Delta \chi^{2}=1$ criterion, for example, arising from partial inconsistencies of the input datasets, using a variety of methods 10,11,98, 101.

\section{Comparisons of PDF sets}

Let us now present a comparison of PDFs and parton luminosities, using the most up-todate versions of the PDF sets. All the PDFs and luminosity comparison plots shown in this document have been obtained with the APFEL on-line PDF plotting tool [102, 103]. We will show comparisons for the ABM12, CT14, HERAPDF2.0, MMHT2014 and NNPDF3.0 NNLO PDF sets. For HERAPDF2.0, the uncertainty shown includes experimental, model, and parametrization uncertainties. For ABM12, we use the $N_{f}=5$ set and the native value of $\alpha_{s}\left(m_{Z}^{2}\right)=0.1132$, since sets for different values of the strong coupling are not available.

\subsection{Parton distributions}

We begin by comparing the gluon and the up quark PDFs, with the corresponding one-sigma uncertainty bands. These are shown in Fig. 3 for $Q=100 \mathrm{GeV}$, normalized to the central value of the CT14 distribution. Similar plots are shown for the $d$ and $\bar{u}$ quarks in Fig. 4 . For each flavor, the left plots compare the three global sets among them, while the right plots compare CT14 with the two sets based on reduced datasets, HERAPDF2.0 and ABM12.

In general, the gluon distributions for CT14, MMHT2014 and NNPDF3.0 agree well, but not perfectly, both in central value and in uncertainty, except at very low $x$ and at high $x$. This level of agreement is reassuring, given the importance of the gluon distribution in the dominant Higgs boson production mechanism. Although each group uses similar, but not identical, data sets, the fitting procedures and tolerances are not the same, and for this reason, exact agreement is not expected. As compared to the global fits, the HERAPDF2.0 gluon PDF is somewhat larger in the $x$ range from $10^{-4}$ to $10^{-2}$ and is substantially smaller for $x \geq 0.1$. Similar considerations apply to ABM12, where their differences as compared to the global sets are due only in part to the difference $\alpha_{s}$ value, with the bulk of the differences due to the use of a FFNS, as discussed in Sect. 2.4 above.

The overlap for the up quark distribution is not as good as for the gluon distribution, so an envelope of CT14, MMHT2014 and NNPDF3.0 would be somewhat broader than the individual predictions. The HERAPDF2.0 and ABM12 up quark distribution are higher than the global sets, especially in the $x$ range from $10^{-2}$ to 0.5 , while HERAPDF2.0 agrees well with CT14 for smaller values of $x$. The CT14, MMHT2014 and NNPDF3.0 down quark distributions, see Fig. 4, are in reasonable agreement with each other over most of the $x$ range, but have slightly different behaviors at high $x$. The HERAPDF2.0 down quark has a different shape, though its uncertainty bands partially overlap with those of the others. ABM12 agrees with the global fits except around $x \sim 0.07$, where it is systematically larger.

Similar conclusions as those drawn from the comparison of $u$ quark PDFs between the three global fits. apply to the $\bar{u}$ quark distributions. Note the blow-up of PDF uncertainties at large $x$ due to the reduced experimental information available in this region. The HERAPDF2.0

$\bar{u}$ quark distribution is generally higher than in the global fits for $x>10^{-4}$ by a substantial 

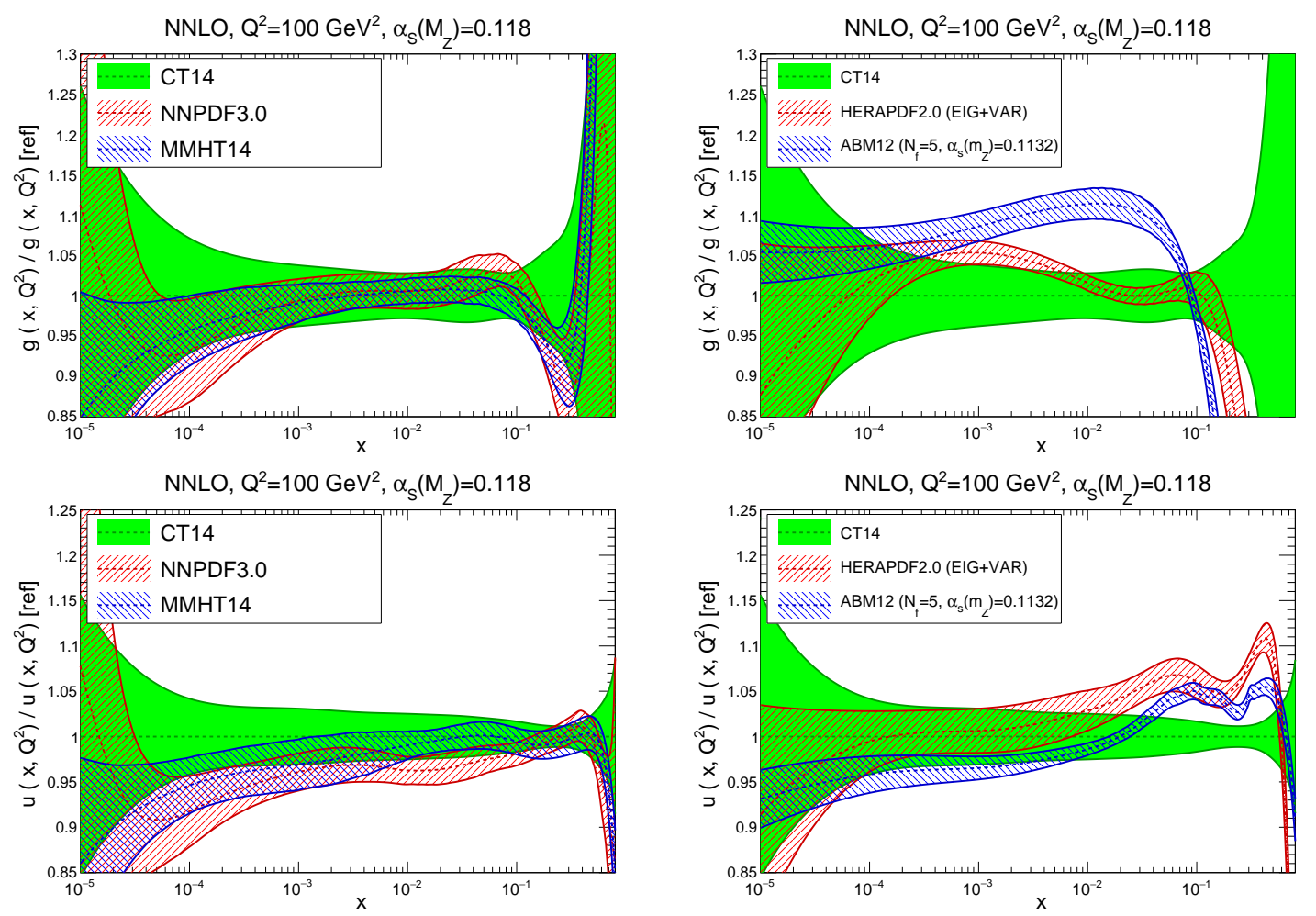

Figure 3: Comparison of the gluon (upper plots) and up quark (lower plots) PDFs from the CT14, MMHT14 and NNNPDF3.0 NNLO sets (left plots) and from the CT14, ABM12 and HERAPDF2.0 sets (lower plots). The comparison is performed at a scale of $Q^{2}=100 \mathrm{GeV}^{2}$, and results are shown normalized to the central value of CT14.

amount (around 10\%). Here ABM12 agrees reasonably with CT14, except for $x \geq 0.1$, where is substantially smaller.

\subsection{PDF luminosities}

It is also instructive to examine the parton-parton luminosities [104], which are more closely related to the predictions for LHC cross-sections. The gluon-gluon and quark-antiquark luminosities, as a function of the invariant mass of the final state $M_{X}$, for a center-of-mass energy of $13 \mathrm{TeV}$ are shown in Fig. 5, where we compare, on the left, the three global fits, NNPDF3.0, CT14 and MMHT14, and, on the right, CT14 with the fits based on reduced datasets, HERAPDF2.0 and ABM12, using for the latter exactly the same settings as in the PDF comparison plots. All results are shown normalized to the central value of CT14, as before. The corresponding comparison for the quark-quark and gluon-quark PDF luminosities is then shown in Fig. 6 .

The luminosity uncertainty ranges tend to blow-up at low invariant masses $\left(M_{X} \leq 50 \mathrm{GeV}\right)$ and high masses $\left(M_{X} \geq 500 \mathrm{GeV}\right.$ for $g g, M_{X} \geq 1 \mathrm{TeV}$ for $q \bar{q}$ and $M_{X} \geq 5 \mathrm{TeV}$ for $\left.q q\right)$, that is, in the regions that are relatively unconstrained in current global PDF fits. The region of intermediate final-state invariant masses can be thought of as the domain for precision physics measurements, where the PDF luminosity uncertainties are less than 5\% (at $68 \% \mathrm{CL}$ ). There 

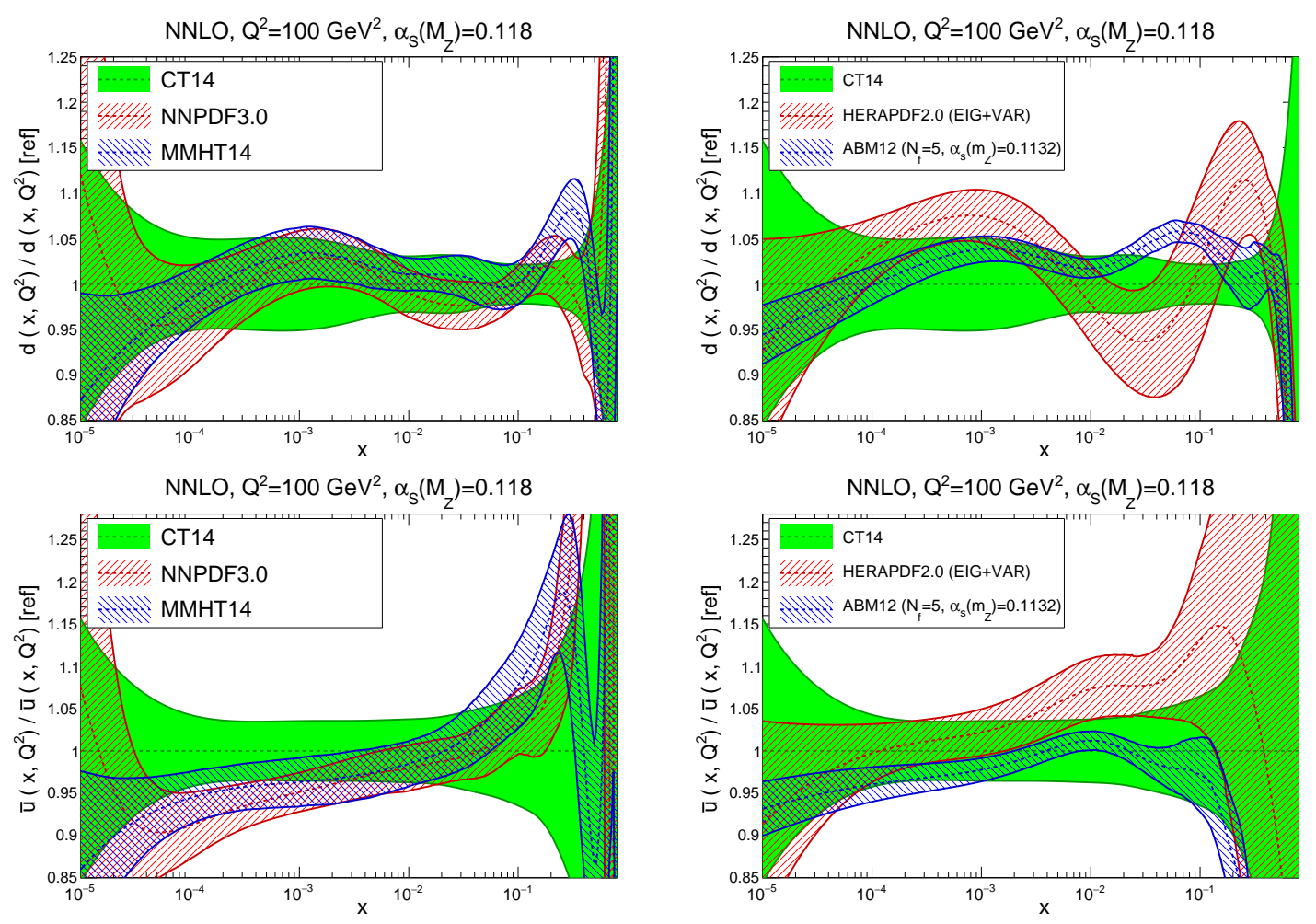

Figure 4: Same as Fig. 3 for the down quark (upper plots) and the anti-up quark (lower plots).

is good agreement among the predictions of CT14, MMHT2014 and NNPDF3.0 for the $g g$ PDF luminosities for this mass range, and in particular for the production of a Higgs boson at $m_{h}=125 \mathrm{GeV}$.

This is an improvement over the situation with the previous generation of PDFs (CT10, MSTW2008 and NNPDF2.3), where, as mentioned previously, the total PDF luminosity uncertainty in the Higgs mass range was more than a factor of 2 larger the uncertainty for any individual PDFs, as a result of differences in central PDFs. Clearly, this leads to a corresponding improvement in agreement between predictions for the cross section $\sigma(g g \rightarrow h)$ computed using these PDF sets. Indeed, as compared to NNPDF2.3, the new NNPDF3.0 prediction decreases by about $2 \%$, the CT14 prediction increases by $1.1 \%$, compared to CT10 while the MMHT14 predictions, as compared to MSTW08, decrease by a small amount (roughly 0.5\%). As discussed in Sect. 2.3, it is difficult to pinpoint a single reason for this improvement in agreement, which most likely arises from a combination of methodological advancements and new experimental constraints in the global fits.

Still considering the $g g$ luminosities, HERAPDF2.0 is in good agreement with the global fits for $M_{X} \leq 0.5 \mathrm{TeV}$, becoming rather softer above this mass, while ABM12 shows strong differences, with a much harder luminosity for $M_{X} \leq 0.5$, and a much softer one above this $M_{X}$ value. For the $q \bar{q}$ luminosity, there is a reasonable agreement between the three global fits; note the blow-up of PDF uncertainties at small and large values of $M_{X}$ because of absence of data. HERAPDF2.0 best-fit luminosity for $q \bar{q}$ is above CT14 by about $5 \%$ in the region of intermediate $M_{X}$, with better agreement at smaller and larger masses, but markedly less 

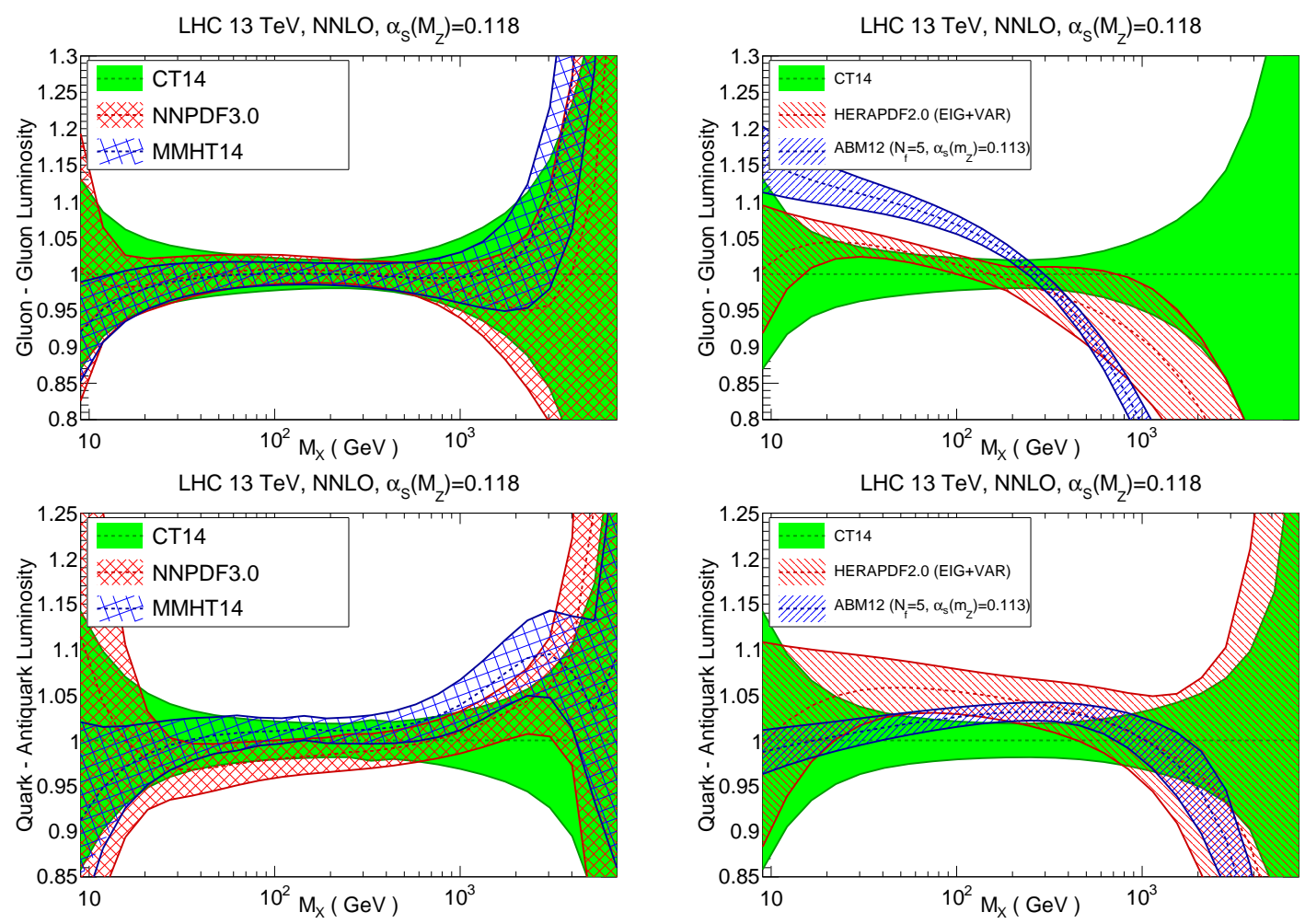

Figure 5: Comparison of the gluon-gluon (upper plots) and quark-antiquark (lower plots) PDF luminosities from the CT14, MMHT14 and NNNPDF3.0 NNLO sets (left plots) and from the NNPDF3.0, ABM12 and HERAPDF2.0 NNLO sets (right plots), for a center-of-mass energy of $13 \mathrm{TeV}$, as a function of the invariant mass of the final state $M_{X}$.

certain. ABM12 is consistent with the global fits except for $M_{X} \geq 2 \mathrm{TeV}$ when it becomes rather softer.

Turning to the quark-quark and gluon-quark PDF luminosities shown in Fig. 6, we see that for the global fits we get consistent results within uncertainties, though the agreement is not quite as good as for the gluon-gluon luminosity, especially in the region between $100 \mathrm{GeV}$ and $1 \mathrm{TeV}$. The luminosities for HERAPDF2.0 and ABM12 in the $q q$ case are harder than those of the global fits, by around $5 \%$ at low masses to up $15 \%$ in the $\mathrm{TeV}$ region, with important phenomenological implications. For the $q g$ luminosity, there is good agreement for the global fits (similar to the $g g$ luminosity) and slightly worse for the PDF sets based on reduced datasets.

\section{Constructing the PDF4LHC15 combination}

As discussed in Sect. 3, we are now in the rather satisfactory position where differences between PDF sets are either better understood or much reduced. However, there is still the question of how best to combine PDF sets even if they are essentially compatible. In this section we motivate and present the updated PDF4LHC prescription for the evaluation of PDF uncertainties at the LHC Run II, discuss the general conditions that PDF sets should satisfy in order to be included in the combination and its future updates, and list the PDF sets which will enter the current prescription. We then describe how the construction of the combined sets based on the 

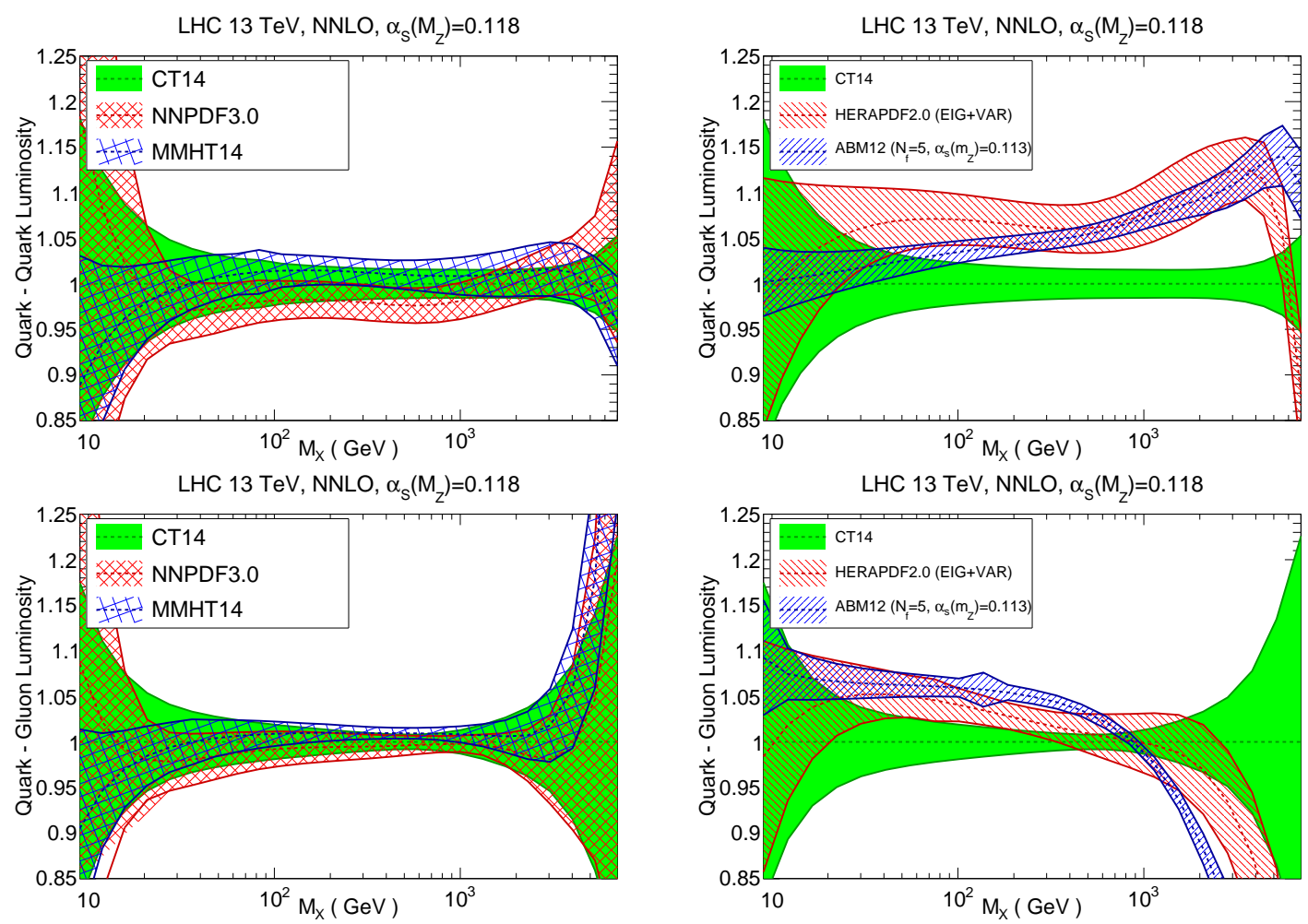

Figure 6: Same as Fig. 5 for the quark-quark (upper plots) and the quark-gluon (lower plots) PDF luminosities.

Monte Carlo method.

\subsection{Usage of PDF sets and their combinations}

We would like first to state that there are three main cases in which PDFs are used in LHC applications:

1. Assessment of the total uncertainty on a cross section based on the available knowledge of PDFs, e.g., when computing the cross section for a process that has not been measured yet (such as supersymmetric particle production cross-sections), or for estimating acceptance corrections on a given observable. This is also the case of the measurements that aim to verify overall, but not detailed, consistency with Standard Model expectations, such as when comparing theory with Higgs measurements.

2. Assessment of the accuracy of the PDF sets themselves or of related Standard Model parameters, typically done by comparing theoretical predictions using individual PDF sets to the most precise data available.

3. Input to the Monte Carlo event generators used to generate large MC samples for LHC data analysis.

In the second case, it is important to always use the PDF sets from the individual groups for predictions. This is especially true in comparisons that involve PDF-sensitive measurements, 
providing information about the PDFs for individual fits. As a rule of thumb, comparisons between QCD or electroweak calculations and unfolded data for Standard Model production processes should be done with individual PDF sets. Similar considerations hold for the third case, since $\mathrm{MC}$ event generators require to be carefully tuned to experimental data using as input specific PDF sets.

However, in the first case above a robust estimate of the PDF uncertainty must accommodate the fact that the individual PDF sets are not identical either in their central values or in their uncertainties. Consequently, an uncertainty based on the totality of available PDF sets must be estimated. Besides acceptance calculations, a contemporary example of this situation is the extraction of the Higgs couplings from LHC data, in which a robust estimate of the overall theoretical uncertainty is essential for probing the nature of the Higgs boson and for identifying possible deviations from the Standard Model expectations. In searches for New Physics particles, such as supersymmetric partners or new heavy $W^{\prime}$ and $Z^{\prime}$ bosons, estimates of the combined uncertainty are also necessary in order to derive robust exclusion limits, or to be able to claim a discovery.

For the applications of type 1, instead of using an envelope, the 2015 PDF4LHC recommendation proposes to take a statistical combination, to be described below, of those PDF sets that satisfy a set of compatibility requirements. While these requirements may evolve with time, for the current combination we select the individual PDF sets that satisfy the following properties:

1. The PDF sets to be combined should be based on a global dataset, including a large number of datasets of diverse types (deep-inelastic scattering, vector boson and jet production, ...) from fixed-target and colliders experiments (HERA, LHC, Tevatron).

2. Theoretical hard cross sections for DIS and hadron collider processes should be evaluated up to two $Q C D$ loops in $\alpha_{s}$, in a general-mass variable-flavor number scheme with up to $n_{f}^{\max }=5$ active quark flavors ${ }^{1}$ Evolution of $\alpha_{s}$ and PDFs should be performed up to three loops, using public codes such as HOPPET [105] or QCDNUM [106], or a code benchmarked to these.

3. The central value of $\alpha_{s}\left(m_{Z}^{2}\right)$ should be fixed at an agreed common value, consistent with the PDG world-average 107. This value is currently chosen to be $\alpha_{s}\left(m_{Z}^{2}\right)=0.118$ at both $\mathrm{NLO}$ and $\mathrm{NNLO}{ }^{2}$ For the computation of $\alpha_{s}$ uncertainties, two additional PDF members corresponding to agreed upper and lower values of $\alpha_{s}\left(m_{Z}^{2}\right)$ should also be provided. This uncertainty on $\alpha_{s}\left(m_{Z}^{2}\right)$ is currently assumed to be $\delta \alpha_{s}=0.0015$, again the same at NLO and NNLO.

The input values of $m_{c}$ and $m_{b}$ should be compatible with their world-average values; either pole or $\overline{\mathrm{MS}}$ masses are accepted.

4. All known experimental and procedural sources of uncertainty should be properly accounted for. Specifically, it is now recognized that the PDF uncertainty receives several contributions of comparable importance: the measurement uncertainty propagated from

\footnotetext{
${ }^{1}$ The combination can also be performed for sets with $n_{f}^{\max }=3$ or $n_{f}^{\max }=4$, provided that these are computed using the $n_{f}^{\max }=5$ sets as boundary conditions for the evolution, see Sect. 6.1

${ }^{2}$ There are arguments that a slightly higher value should be taken at NLO than at NNLO, but the majority request from $\mathrm{PDF}$ users is to have a common value for both orders.
} 
the experimental data, uncertainties associated with incompatibility of the fitted experiments, procedural uncertainties such as those related to the functional form of PDFs, the handling of systematic errors, etc. Sets entering the combination must account for these through suitable methods, such as separate estimates for additional model and parametrization components of the PDF uncertainty [9], tolerance [6, 10], or closure tests [11].

Following the needs of precision physics at the LHC, future updates of the PDF4LHC recommendations might be based on a different set of conditions. For instance, in addition to the above PDF sets, one might be required to provide fits in a range of $m_{c}$ and $m_{b}$ values, or to provide direct evidence of the ability to describe, reasonably accurately, a wide variety of different data types that constrain PDFs of different flavour and in different kinematic regions. In the future, with the progress in combination techniques, it might be possible to relax the first requirement, and also include in the combination PDF sets based on datasets of a different size by using suitable weighted averaging techniques — note that such a weighted averaging would have to account for the fact that different data affect different PDFs and kinematic regions, and thus the weights chosen cannot be the same for all PDF flavours.

The existing PDF sets which satisfy all of these requirements at present have been identified as CT14, MMHT2014 and NNPDF3.0; no other publicly available PDF sets currently appears to satisfy all conditions.

\subsection{Statistical combination of PDF sets}

Currently, two different representations of PDF uncertainties are being used 12: the Monte Carlo representation 108 110, in which the probability distribution of PDFs is given as an ensemble of replicas, whose mean and standard deviation provide respectively central value and uncertainty, and the Hessian representation 99, in which a central PDF is given, along with error sets, each of which corresponds to an eigenvector of the covariance matrix in parameter space.

In Ref. [101, a comparison of the Hessian and the Monte Carlo methods was made within the MSTW framework, showing that they provide compatible representations of PDF uncertainties, and in particular lead to the same uncertainties when used to determine PDFs from known pseudo-data. Also, a way of obtaining a Monte Carlo representation from starting Hessian representation was presented, and the accuracy of the Hessian to Monte Carlo conversion was explicitly demonstrated. As will be discussed in the next section, recently two methods to perform the inverse conversion, namely transforming MC sets into a Hessian representation, have also been developed [111, 112].

These developments make possible a statistical combination of different PDF sets and their predictions, as originally outlined in Ref. [2]: if different PDF sets are assumed to be equally likely representations of an underlying PDF probability distribution, they can be combined by simply taking their unweighted average. This in turn can be done simply by generating equal numbers of Monte Carlo replicas from each input PDF set, and then merging these replica sets of equal sizes. For the Hessian PDF sets such as CT or MMHT, the Monte-Carlo replicas are generated by sampling along each eigenvector direction, assuming a Gaussian distribution. Alternatively, a weighted average would correspond to taking different number of replicas from the various sets sets entering the combination. First examples of this application for LHC cross 
sections were given in Ref. [3]. A detailed overview of how the MC method has been used to construct the PDF4LHC 2015 combined sets will be presented in the next section.

Clearly, results obtained in this way are less conservative than those obtained from an envelope: they correspond to the assumption that different PDF determinations are statistically distributed instances of an underlying probability distribution, rather than instances of a probability distribution affected by unknown underlying sources of systematics. Such a combination method appears therefore to be adequate when results are compatible, or differences are understood, as is the case now.

\section{Implementation and delivery of the PDF4LHC15 PDFs}

In this section we discuss the technical construction of the new PDF4LHC prescription, presented in Sect. 4, and the delivery of the combined PDFs based on it. Readers interested in the more practical question of how to use the PDF4LHC15 combination in their specific analysis can jump directly to Sect. 6 .

Unlike the previous PDF4LHC prescription, in which the task of combining the PDF uncertainties was left to the user, with the current PDF4LHC prescription, several pre-packaged PDF sets that already combine the uncertainties will be constructed and delivered. The combined sets are based on a statistical combination of the CT14, MMHT2014 and NNPDF3.0 PDF sets, as discussed in Sect. 4.2. This combination leads to a "prior" Monte Carlo set with $N_{\text {rep }}=900$ replicas, to be referred to as either MC900 or PDF4LHC15_prior in the following discussion. Such a large replica set would be unmanageable; however, various methods have been proposed recently to deal with it, which we collectively refer to as reduction methods. Usage of these methods will allow for a compact delivery of the combined PDF sets.

The idea of producing a unified PDF set by combining various individual sets was first suggested in Ref. [111], based on the idea of refitting a suitable functional form to a combined set of Monte Carlo replicas, thereby leading to a representation of the starting Monte Carlo probability distribution in terms of Hessian error sets in parameter space (META-PDFs). This then realizes the dual goal of producing a combination, and then reducing the number of PDF error members to a manageable size. More recently, other reduction methods were suggested, with the similar goal of turning the starting combined Monte Carlo sample into a more manageable representation. The Monte Carlo compression method [113] selects a subset of the original replica sample which reproduces its statistical features with minimal information loss, thereby keeping the MC representation, but with a smaller number of replicas (CMC-PDFs). The Monte Carlo to Hessian conversion method [112 turns a set of Monte Carlo replicas into Hessian error sets by representing the covariance matrix in the space of PDFs on a discrete set of points in $x$ as a linear combination of the replicas, by means of a Singular Value Decomposition followed by Principal Component Analysis (MCH-PDFs).

In this section, we will first present the Monte Carlo combination of the PDF sets and, in particular, determine the number of replicas that is necessary in order to achieve a faithful description of the combined set. We will then review each of the three reduction and delivery methods: CMC (Monte Carlo), META (Hessian) and MCH (Hessian), and, in each case, identify the size of the corresponding reduced PDF sets that optimizes the specific features of the method. These three sets will be adopted for delivery of the combined PDF4LHC15 PDF sets. Finally, we will compare and benchmark the PDF sets obtained with the three different 
reduction strategies, both at the level of parton distributions and of LHC observables.

\subsection{The Monte Carlo combination of PDF sets}

The first step in the construction of a Monte Carlo statistical combination $2,3,101,111,113,114$ is the transformation of the Hessian PDF sets into Monte Carlo PDF sets using the WattThorne method [101]. Once all sets have been turned into a Monte Carlo representation, the combination is simply built by adding together an equal number of replicas from each set. The generation of the Monte Carlo replicas is based on the symmetric Thorne-Watt formula, see Eq. (22) in Ref. [115], as implemented in the LHAPDF6 code [115. This has been cross-checked with the independent code of the MP4LHC package, finding excellent agreement.

In Fig. 7 we show the comparison of the PDFs from the Monte Carlo combination of CT14, MMHT14 and NNPDF3.0 for a different number of MC replicas, $N_{\text {rep }}=300,900$ and 1800, referred in the following as MC300, MC900 and MC1800. The error bands correspond to one standard deviation from the mean value. We see that, while between $N_{\text {rep }}=300$ and $N_{\text {rep }}=900$ some visible, albeit small, changes are observed, results stabilize if a yet larger number of replicas is used. We conclude that $N_{\text {rep }}=900$ (i.e. 300 replicas from each of three individual sets) is an adequate number for the combination, and this is the value that we will adopt henceforth as a default for our prior. Therefore, the prior combined set PDF4LHC15_prior coincides with the MC900 set.

In Fig. 8 we compare the MC900 combined PDF set with the three individual PDF sets, CT14, MMHT14 and NNPDF3.0, at NNLO, for $\alpha_{s}\left(m_{Z}^{2}\right)=0.118$ at $Q^{2}=10^{4} \mathrm{GeV}^{2}$. Results are normalized to the central value of MC900. Because of the good consistency of the PDF sets which enter the combination, the combined uncertainty is not much larger than that of individual PDF sets. A somewhat more significant increase observed in some cases, for instance, for the strange $s(x, Q)$ around $x \simeq 0.05$, due to the larger differences between the individual PDF sets. By construction, the uncertainty on the statistical combination remains always smaller than that of the envelope method.

In Fig. 9 we show the probability distribution for MC900 compared to that of the individual input PDF sets. Results are shown for the gluon at $x=0.01$, the up quark at $x=5 \cdot 10^{-5}$, the down antiquark at $x=0.2$ and the strange PDF at $x=0.05$. The histograms represent the probability per bin of each PDF value, quantified by the number of replicas that fall into that bin. For comparison, a Gaussian distribution with the same mean and variance as that of the MC900 histogram is also plotted. The combination appears to be generally Gaussian to good approximation, though some features of the combined distribution provide evidence of deviation from Gaussian behaviour, for example a non-vanishing skewness in the $\bar{d}(x, Q)$ and $s(x, Q)$ distributions.

The non-Gaussian features of the MC combination can also be observed for specific LHC cross-sections, in particular for extreme kinematic regions or for those processes that involve PDF combinations affected by large uncertainties. As an illustration, in Figs. 10 and 11 we show the probability distribution for MC900 using the Kernel Density Estimation (KDE) method 113 for two specific observables. First of all, the $W+$ charm differential cross-section in the lepton rapidity from the CMS measurement [116] in the range $\eta_{l} \in[2.1,2.5]$, and then the forward Drell-Yan process in dileptons from the LHCb measurement [36] in the range for $\eta_{l} \in[4.2,4.5]$. These probability distributions are clearly non-Gaussian: double-hump structure for $W+$ charm; significant skewness for LHCb forward Drell-Yan. In the same plot, 
$g(x, Q)$ at $Q=8 \mathrm{GeV}, \pm 1$ std. dev.

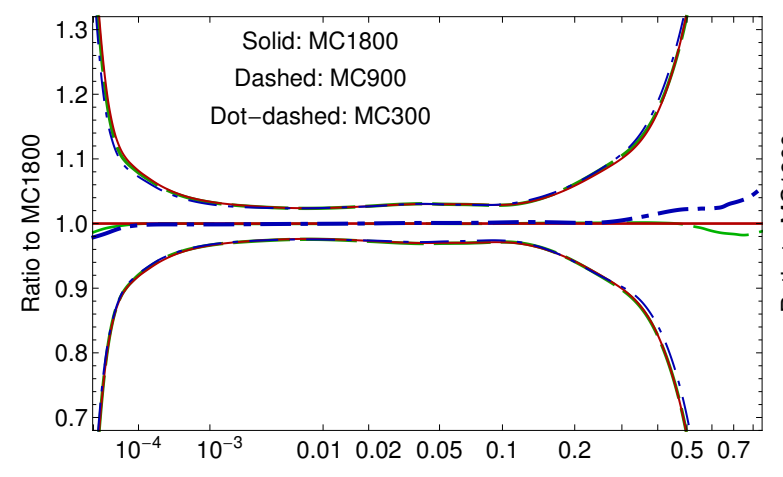

$\bar{u}(\mathrm{x}, \mathrm{Q})$ at $\mathrm{Q}=8 \mathrm{GeV}, \pm 1$ std. $\mathrm{dev}$.
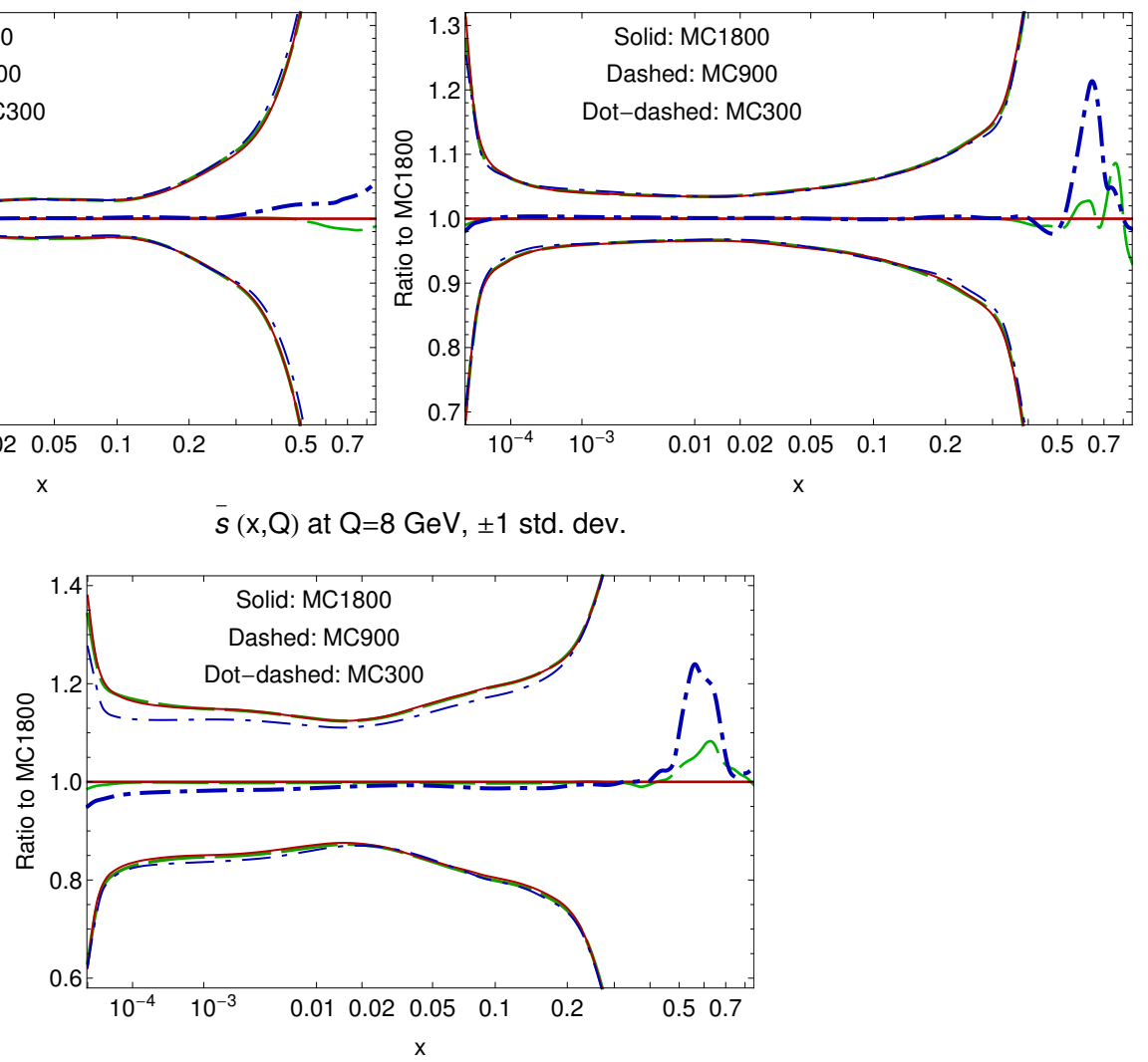

Figure 7: Comparison of central values and uncertainties for the MC combination of CT14, MMHT14 and NNPDF3.0 for different values of $N_{\text {rep }}, 300,600$ and 900, denoted by MC300, MC900 and MC1800 respectively.

results obtained after applying two reduction techniques are also shown, to be discussed below.

The non-Gaussian features for $W$ +charm production shown in Fig. 10 are related to the different assumptions on the parameterization of the strange PDF adopted by the various groups. For instance, the MMHT14 analysis allows the $D \rightarrow \mu$ branching ratio to be determined from the fit, increasing the associated uncertainties in the strange PDF. Here the usefulness of the present combination method is clear, allowing to combine in a statistically consistent way the different assumptions used by each group (under the assumption that each of the various choices has equal prior likelihood - for the sets that enter the combination).

The discussion above refers to the combination of PDF sets for a common value of $\alpha_{s}\left(m_{Z}^{2}\right)=$ 0.118. In addition, we need to provide combined sets for the values $\alpha_{s}\left(m_{Z}^{2}\right)=0.1165$ and $\alpha_{s}\left(m_{Z}^{2}\right)=0.1195$ in order to be able compute the combined PDF $+\alpha_{s}$ uncertainty. These varying- $\alpha_{s}$ PDF sets can be constructed from a simple average of existing sets from the individual groups. If we denote by $q^{\text {pdf4lhc }}$ the central value of combined PDF4LHC set for a specific quark flavour (or the gluon), then the sets with different values of $\alpha_{s}$ can be constructed 

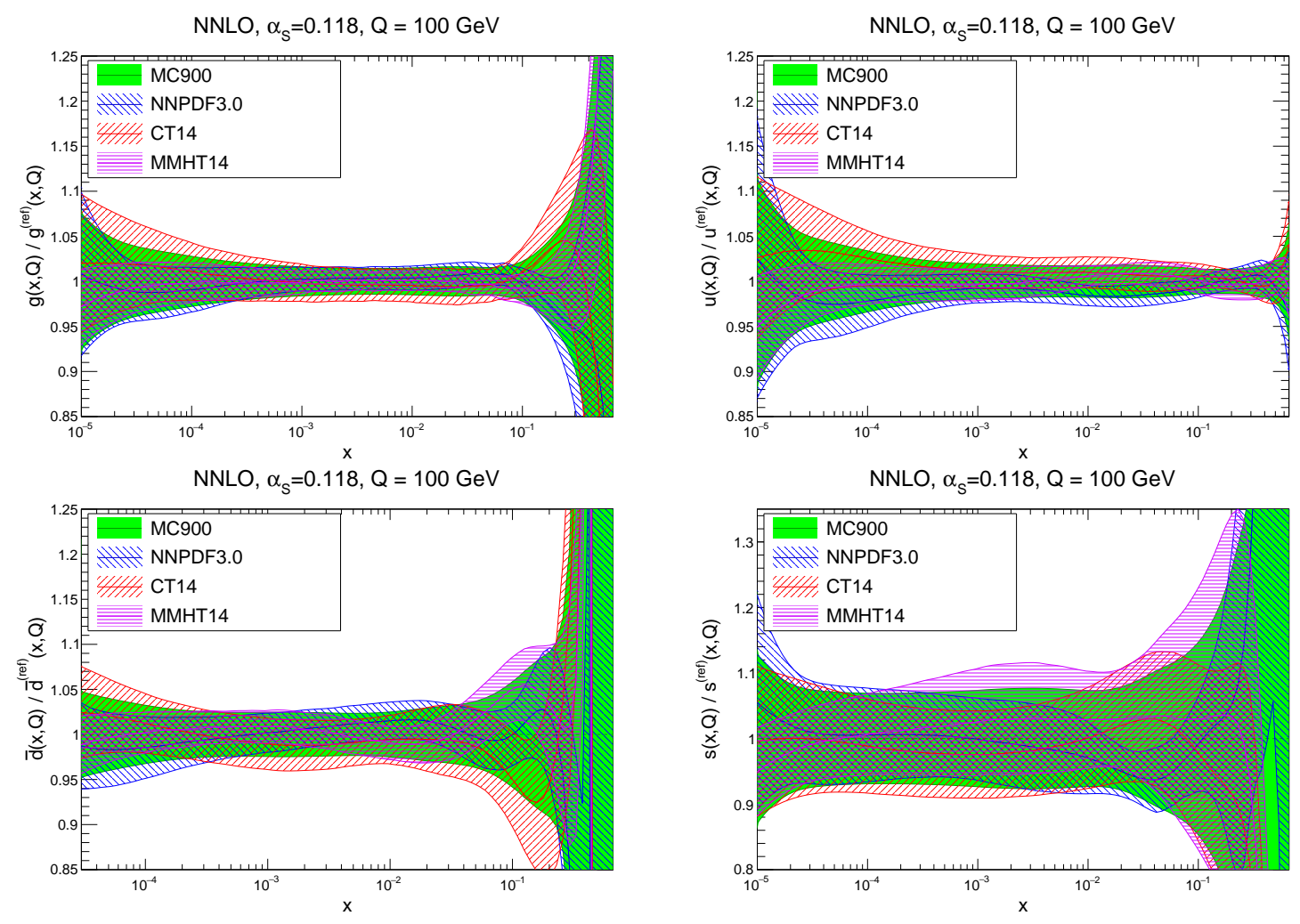

Figure 8: Comparison of the MC900 PDFs with the sets that enter the combination: CT14, MMHT14 and NNPDF3.0 at NNLO. We show the gluon and the up, anti-down and strange quarks at $Q=100$ $\mathrm{GeV}$. Results are normalized to the central value of MC900.

as follows:

$$
\begin{aligned}
q^{\mathrm{pdf} 4 \mathrm{lhc}}\left(\alpha_{s}=0.1165\right) & =\frac{1}{6}\left(q^{\mathrm{mmht}}\left(\alpha_{s}=0.116\right)+q^{\mathrm{mmht}}\left(\alpha_{s}=0.117\right)\right) \\
& +\frac{1}{6}\left(q^{\mathrm{ct}}\left(\alpha_{s}=0.116\right)+q^{\mathrm{ct}}\left(\alpha_{s}=0.117\right)\right) \\
& +\frac{1}{6}\left(q^{\mathrm{nnpdf}}\left(\alpha_{s}=0.115\right)+q^{\mathrm{nndf}}\left(\alpha_{s}=0.118\right)\right)
\end{aligned}
$$

and

$$
\begin{aligned}
q^{\mathrm{pdf} 4 \mathrm{lhc}}\left(\alpha_{s}=0.1195\right) & =\frac{1}{6}\left(q^{\mathrm{mmht}}\left(\alpha_{s}=0.119\right)+q^{\mathrm{mmht}}\left(\alpha_{s}=0.120\right)\right) \\
& +\frac{1}{6}\left(q^{\mathrm{ct}}\left(\alpha_{s}=0.119\right)+q^{\mathrm{ct}}\left(\alpha_{s}=0.120\right)\right) \\
& +\frac{1}{6}\left(q^{\mathrm{nnpdf}}\left(\alpha_{s}=0.121\right)+q^{\mathrm{nnpdf}}\left(\alpha_{s}=0.118\right)\right)
\end{aligned}
$$

with $q^{\text {mmht }}, q^{\text {ct }}$ and $q^{\text {nnpdf }}$ the corresponding central values of the MMHT14, CT14 and NNPDF3.0 sets for those specific values of $\alpha_{s}\left(m_{Z}^{2}\right)$. We have verified that other possible ways of constructing these sets (such as different interpolation options) do not change the result in any appreciable way. 

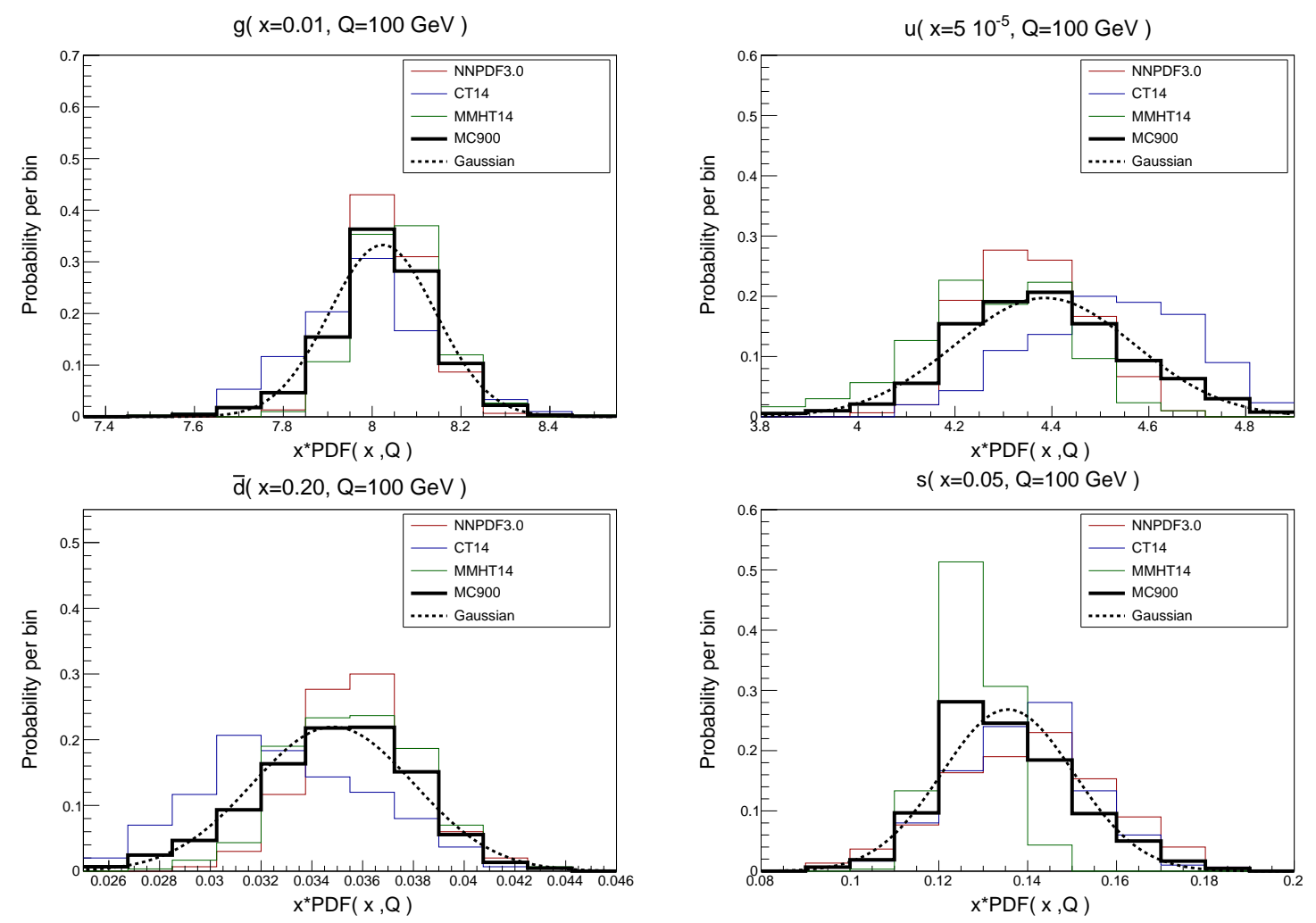

Figure 9: Probability distribution of MC900, compared to that of the individual input sets CT14, MMHT14 and NNPDF3.0 NNLO. Results are shown for the gluon at $x=0.01$, the up quark at $x=5 \cdot 10^{-5}$, the down antiquark at $x=0.2$ and the strange PDF at $x=0.05$. All PDFs have been evaluated at $Q=100 \mathrm{GeV}$. The histograms represent the probability per bin of each PDF value, quantified with the number of replicas that fall into that bin. For comparison, a Gaussian distribution with the same mean and variance of the MC900 histogram is plotted.

\subsection{The Monte Carlo reduction method: CMC-PDFs}

Compressed Monte Carlo PDFs (CMC-PDFs) [113] are determined by using a compression algorithm, that, starting from a MC prior with $N_{\text {rep }}$ replicas, determines the set of $\widetilde{N}_{\text {rep }}<N_{\text {rep }}$ replicas that most faithfully reproduce the original probability distribution in terms of central values, variances, higher moments and corrections. Therefore with the CMC-PDFs one ends up with a Monte Carlo representation of the original MC900 combination but based on a much reduced number of replicas (about an order of magnitude reduction), with minimal information loss.

The compression algorithm is based on the minimization of a figure of merit,

$$
\mathrm{ERF}=\sum_{k} \frac{1}{N_{k}} \sum_{i}\left(\frac{C_{i}^{(k)}-O_{i}^{(k)}}{O_{i}^{(k)}}\right)^{2},
$$

where $k$ runs over the number of statistical estimators used to quantify the distance between the original and compressed distributions, $N_{k}$ is a normalization factor, $O_{i}^{(k)}$ is the value of the estimator $k$ (for example, the mean or the variance) computed at the generic point $\left(x_{i}, Q_{i}\right)$ 


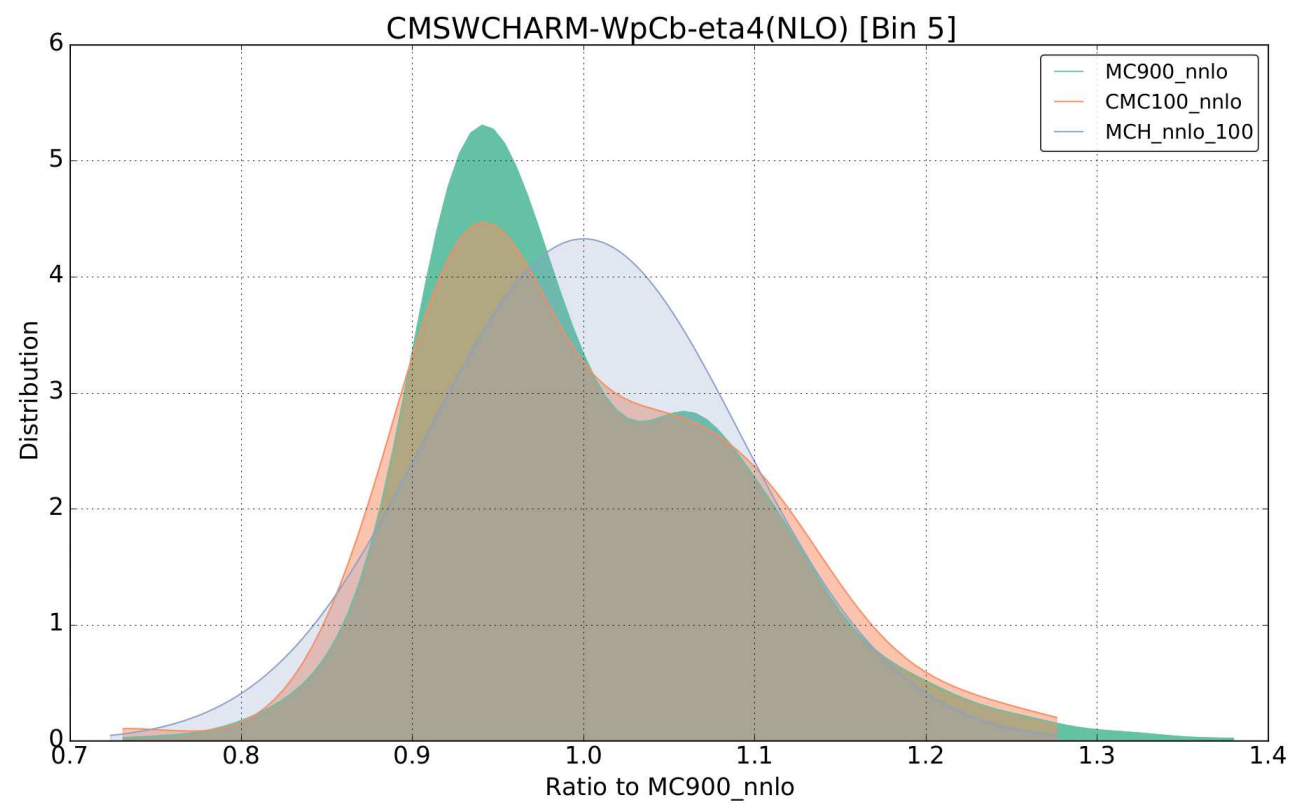

Figure 10: The probability distribution computed with the Kernel Density Estimation method for the MC900 prior and the CMC100 and MCH100 reduced sets, for the most forward bin of the CMS $W+$ charm differential cross-section measurement [116].

and $C_{i}^{(k)}$ is the corresponding value of the same estimator in the compressed set. The various contributions to Eq. (3) include the mean, variance, skewness, kurtosis, the Kolmogorov distance and the correlations between PDFs. The minimization is performed using Genetic Algorithms. The main advantage of the CMC-PDF method is that not only the central value and variance of the original distribution are reproduced, but also its higher moments: this is of course crucial when the underlying probability distribution is non-Gaussian, as illustrated in Figs. 10 and 11.

The quality of the compression has been extensively validated in Ref. [113] at the level of PDFs and of LHC cross-sections, including their correlations; the general conclusion is that $N_{\text {rep }} \simeq 100$ replicas are enough to reproduce all relevant statistical estimators of the original combined PDF set and can be reliably used in LHC phenomenology: a comparative quantitative assessment will be given below.

The choice of estimators $O_{i}^{(k)}$ included in the figure of merit Eq. (3) is a compromise between the goal of reproducing reasonably well both the low moments, and specifically the mean and standard deviation of the distributions (which fully determine the Gaussian approximation), and the higher moments of the distribution. Specifically, if only the Kolmogorov distance were included, all moments would be reproduced equally well, while if only the mean and standard deviation were included, only these would be optimized. Therefore, in general there is a tradeoff in accuracy: the CMC-PDFs will perform slightly worse in reproducing the central values and variances of the prior as compared to a Hessian reduction, but the advantage of keeping the non-Gaussian features which are missed by the latter case.

The CMC100 PDFs have been used to construct the default Monte Carlo representation of the PDF4LHC combined sets that are made available on LHAPDF6: 


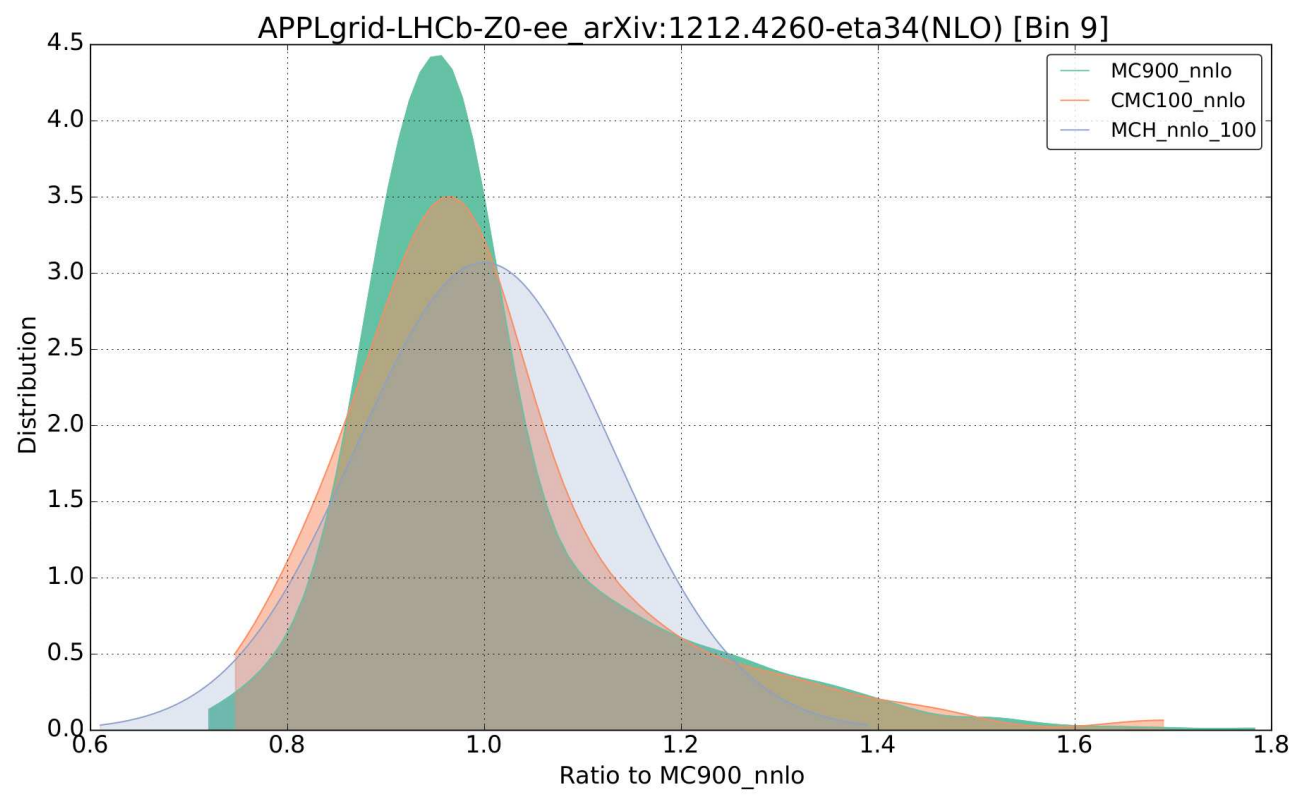

Figure 11: Same as Fig. 10 for forward Drell-Yan measurement from LHCb 36].

PDF4LHC15_nlo_mc

PDF4LHC15_nnlo_mc

In addition, the compressor code use to generate the CMC-PDF sets is also publicly available from

https://github.com/scarrazza/compressor

together with the corresponding user documentation. For completeness, Sect. 6.2 reports the formulae that should be used to compute the PDF and PDF $+\alpha_{s}$ uncertainties whenever the PDF4LHC15_mc sets are used.

\subsection{Hessian reduction methods}

A Hessian representation of PDF uncertainties has certain advantages and disadvantages in comparison to a Monte Carlo representation. The main disadvantage is that a Hessian representation assumes that the underlying probability distribution is multi-Gaussian (though in general possibly asymmetric, i.e. with different upper and lower uncertainties). The main advantage is that Hessian uncertainties can be treated as nuisance parameters, and thus on the same footing as other nuisance parameters: this can be useful in analysis, for example for the determination of PDF-induced correlations in large datasets, and also when using profiling in order to understand the dominant PDF contributions to a given process.

Two techniques have been suggested to turn a Monte Carlo PDF set into a Hessian set: META-PDF and MCH-PDFs. By carefully tuning the number of Hessian sets which are used, these techniques also provide a suitable reduction method. Clearly, when choosing the number of eigenvectors $N_{\text {eig }}$ to be included in a Hessian representation of PDF errors there is a trade-off between speed and accuracy. The optimal number of $N_{\text {eig }}$ thus depends then on the specific 
application, i.e., whether speed or accuracy is the most important consideration. We will now present each of the two methods, and then discuss the choice of the number of PDF error members.

\subsubsection{META-PDFs}

The meta-analysis of Ref. [111] was the first method proposed for reducing the number of Monte-Carlo replicas in the combined PDF ensemble. The META analysis starts from the Monte Carlo representation of the input PDF set. Then, each MC replica is re-fitted using a flexible "meta-parametrization", so that all input replicas are cast into a common parametrized form. The probability distribution is examined as a function of parameters $a_{i}$ of the metaparametrizations. The least and best constrained combinations of $a_{i}$ are found by diagonalization of the covariance matrix on the PDF parameter space.

From this information, one can construct Hessian error PDFs that reproduce intervals of given confidence along each poorly constrained direction, centered on the average PDF set of the input ensemble. In contrast, error PDFs corresponding to displacements along wellconstrained directions contribute little to the total PDF uncertainty and can be discarded. In the end, one obtains a central PDF, corresponding to an average of the input replicas; as well as an ensemble consisting of a relatively small number $N_{\text {eig }}$ of Hessian eigenvectors, reproducing the principal components of the original covariance matrix.

Introduction of meta-parametrizations thus leads to a fairly intuitive method for combination, which reduces to averaging of discrete parameters and diagonalization of a covariance matrix in a quasi-Gaussian approximation. While the choice of the functional form for the meta-parametrizations is not unique, it does not bias the reduced META-PDFs in practice, which has been verified by trying various parametrization forms and fitting procedures.

In the current study, we construct a META-PDF combination with one central and $N_{\text {eig }}=$ 30 symmetric eigenvectors according to the following procedure. We start from the same prior

MC900, see Sect. 5.1 as done in the other reduction methods. Each PDF replica $f_{\alpha}^{(k)}\left(x, Q_{0}\right)$ is then re-fitted, at a given input scale $Q_{0}$, using the form

$$
\Phi_{\alpha}^{(k)}(x ;\{a\})=f_{\alpha}^{(0)}\left(x, Q_{0}\right)\left(1+\sum_{i=1, \ldots} a_{\{\alpha, i\}}^{(k)} b_{i}(x)\right),
$$

where $f_{\alpha}^{(0)}\left(x, Q_{0}\right)$ is the average PDF set for flavor $\alpha$. The basis functions are

$$
b_{i}(x)=\left\{\ln (x), \ln (1-x), \mathcal{B}_{14, i-2}(x)\right\},
$$

for $i=1,2, \ldots$ and include Bernstein polynomials of degree $n=14$,

$$
\mathcal{B}_{n, i}(x)=\left(\begin{array}{c}
n \\
i
\end{array}\right) x^{i}(1-x)^{n-i}
$$

The parameters $a_{\{\alpha, i\}}^{(k)} \equiv a_{l}^{(k)}$ are zero for the central PDF set, but vary for each of the MonteCarlo replicas. They are chosen to minimize a metric function $E$ on a grid $\left\{x_{n}\right\}$ of momentum fraction values in the interval $x=\left[3 \cdot 10^{-5}, 0.9\right]$ at the initial scale $Q_{0}$ :

$$
E\left[f_{\alpha}^{(k)}\left(x, Q_{0}\right), \Phi_{\alpha}^{(k)}(x ;\{a\})\right]=\sum_{x \text { grid }}\left[\frac{\ln f_{\alpha}^{(k)}\left(x_{n}, Q_{0}\right)-\ln \Phi_{\alpha}^{(k)}\left(x_{n} ;\{a\}\right)}{\delta\left(\ln f_{\alpha}\left(x_{n}, Q_{0}\right)\right)}\right]^{2}
$$


where $\delta\left(\ln f\left(x, Q_{0}\right)\right) \equiv \delta f\left(x, Q_{0}\right) / f\left(x, Q_{0}\right)$, and $\delta f\left(x, Q_{0}\right)$ is the symmetric PDF uncertainty of $f\left(x, Q_{0}\right)$.

The value of $Q_{0}$ where the MC replicas are parametrized is taken to be $Q_{0}=8 \mathrm{GeV}$, well above the bottom-quark mass $m_{b}$, where all input heavy-quark schemes lead to close predictions. Thus we fit $9 \times 16=144$ parameters for 9 independent PDF flavors at $Q_{0}$, and setting $\bar{c}\left(x, Q_{0}\right)=c\left(x, Q_{0}\right), \bar{b}\left(x, Q_{0}\right)=b\left(x, Q_{0}\right)$ for simplicity. Small differences between sea quarks and antiquarks induced purely by NNLO evolution effects are negligible here, given that $Q_{0}$ is still relatively low.

The covariance matrix in the space of PDF parameters is computed according to

$$
\operatorname{cov}\left(a_{l}, a_{m}\right)=\frac{N_{\text {rep }}}{N_{\text {rep }}-1} \sum_{k=1}^{N_{\text {rep }}} a_{l}^{(k)} a_{m}^{(k)},
$$

and diagonalised by an orthogonal transformation $O$ :

$$
O_{l l^{\prime}} \cdot \operatorname{cov}\left(a_{l^{\prime}}, a_{m^{\prime}}\right) \cdot O_{m^{\prime} m}^{T}=\lambda_{l} \delta_{l m} .
$$

The eigenvalues $\lambda_{l}$ of the covariance matrix are positive-definite. The eigenvectors are associated with the parameters $a_{l}^{\prime} \equiv O_{l m} a_{m}$. We can then reasonably interpret $\sqrt{\lambda_{l}}$ to be the width of the effective Gaussian distribution describing $a_{i}^{\prime}$, and neglecting any asymmetry of this distribution.

To construct the META ensemble we select the largest, or principal, eigenvalues $\lambda_{l}$, and discard the eigenvectors associated with well-constrained $a_{i}^{\prime}$. For the discarded directions, the respective $a_{i}^{\prime}$ are set to zero, their average value; the number $N_{\text {eig }}$ of eigenvectors for the final META-PDFs thus reduces below the original number of 144. Using the META representation, a standard deviation on a quantity $X$ can be computed using the usual Hessian symmetric master formula,

$$
\delta X=\sqrt{\sum_{k=1}^{N_{\text {eig }}}\left(X^{(k)}-X^{(0)}\right)^{2}},
$$

where $X^{(0)}$ and $X^{(k)}$ are the predictions for the central and $k$-th eigenvector of the META ensemble. This is a symmetrized master formula, which provides an estimate of the $68 \%$ CL uncertainty at all $Q$ values, providing the DGLAP evolution of all input PDFs is numerically consistent ${ }^{3}$ In this approach, common DGLAP evolution settings are used for all the META eigenvectors using the HOPPET program 105].

The Hessian META uncertainty provides a lower estimate for the MC900 uncertainty. For example, the META-PDF set with $N_{\text {eig }}=100$ symmetric eigenvectors (denoted by META100 in the following) exhibits PDF uncertainties closer to the MC900 uncertainty than the META30 uncertainty (the META-PDF set with $N_{\text {eig }}=30$ symmetric eigenvectors), but requires the evaluation of a larger number of eigenvectors. We find that the META30 combination efficiently

\footnotetext{
${ }^{3}$ Agreement between the codes for numerical DGLAP evolution at NNLO has been confirmed by a benchmarking exercise in the appendix of [111. An already small difference between NNLO evolution of MSTW'08 grid files and HOPPET was further reduced in the MMHT14 release. At NLO, the NNPDF3.0 sets evolve according to a truncated solution of the DGLAP equation, which deviates marginally from the evolution of the CT14 and MMHT14 sets. We apply a correction to the LHAPDF grid files of the META-NLO ensemble after the combination to compensate for this weak effect.
} 
captures information about the lowest two (Gaussian) moments of the original MC900 distribution, which are the most robust and predictable. The number $N_{\text {eig }} \simeq 25-30$ corresponds to about the minimal number of parameters that are needed to describe PDF degrees of freedom in at least three independent $x$ regions (small, intermediate, and large $x$ ) for the nine physical flavors.

In a sense, META30 provides a "minimal" estimate of the PDF uncertainty, so that "the true uncertainty must be at least as much with good confidence". META100, on the other hand, provides a more complete estimate for the given prior, by including subleading contributions that are also more prone to variations.

The META30 PDFs are parametrized at a starting scale of $Q_{0}=8 \mathrm{GeV}$, above which one can neglect differences in the treatment of heavy-quark flavors in the CT14, MMHT2014, and NNPDF3.0 PDF sets. This is sufficient for computing hard-scattering cross sections for typical LHC observables using the $N_{f}=5$ massless approximation. However, in those calculations that match the finite-order cross sections with initial-state parton showers, knowing the PDFs at scales below $8 \mathrm{GeV}$ may also be necessary in order to calculate the Sudakov form factors. For these specific applications, the META30 PDFs have been extended down to $Q_{\text {min }}=1.4$ $\mathrm{GeV}$ by backward DGLAP evolution, and the stability of this low- $Q$ extension was validated using several tests. The resulting uncertainties at low $Q$ are in good agreement with those from the the MCH-100 PDFs. In the publicly provided LHAPDF grids for the PDF4LHC15_30 set, the whole range of $Q \geq 1.4 \mathrm{GeV}$ is covered; the transition across the fitting scale of $8 \mathrm{GeV}$ is invisible to the user. Only above $8 \mathrm{GeV}$ the treatment of heavy flavors is fully consistent; below this scale the combined PDFs are suitable for specific calculations, such as those involving parton showering generators.

\subsubsection{MCH-PDFs}

The mc2Hessian algorithm for construction of a Hessian representation of a Monte Carlo PDF set uses the replicas themselves as a linear expansion basis. This idea was realized in two different ways in Ref. 112. The first method first identifies the region where the Gaussian approximation is valid, and uses Genetic Algorithms to identify the optimal expansion basis. The second method uses Singular Value Decomposition (SVD) to represent the covariance matrix on a basis of replicas.

This second method is the preferred one if the goal is achieving an optimal reproduction of the covariance matrix, and it is the method which is reviewed here. In this method, the multiGaussian distribution of replicas is viewed as a distribution of deviations of PDFs from their central value. If $N_{\text {pdf }}$ independent PDFs $f_{\alpha}^{(k)}\left(x_{i}, Q\right), \alpha=1, \ldots, N_{\text {pdf }}$, at scale $Q$ are sampled on a fine enough grid of $N_{x}$ points in $x_{i}$, the deviations from the central $\operatorname{PDF} f_{\alpha}^{(0)}\left(x_{i}, Q\right)$ can

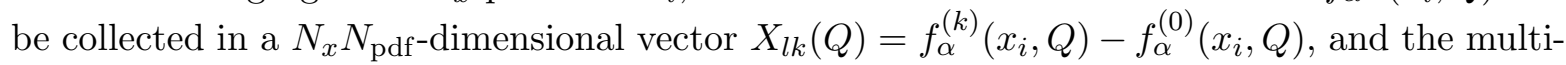
Gaussian is fully determined by the corresponding $N_{x} N_{\text {pdf }} \times N_{x} N_{\text {pdf }}$ covariance matrix. This, in turn, is represented as a linear combination of the starting replicas $f_{\alpha}^{(k)}\left(x_{i}, Q\right), k=1, \ldots, N_{\text {rep }}$.

This is done in the following way. The set of vectors $X$ of deviations from the central value, for each replica,

$$
X_{l k}(Q)=f_{\alpha}^{(k)}\left(x_{i}, Q\right)-f_{\alpha}^{(0)}\left(x_{i}, Q\right),
$$

where $\alpha$ labels PDFs, $i$ points in the $x$ grid, $l \equiv N_{x}(\alpha-1)+i$ runs over all $N_{x} N_{\text {pdf }}$ grid points, $k$ runs over all the $N_{\text {rep }}$ MC replicas, and $f_{\alpha}^{(0)}\left(x_{i}, Q\right)$, can be viewed as a $N_{x} N_{\text {pdf }} \times N_{\text {rep }}$ 
rectangular matrix. The $N_{x} N_{\text {pdf }} \times N_{x} N_{\text {pdf }}$ covariance matrix can then be written as

$$
\operatorname{cov}_{l l^{\prime}}(Q)=\frac{1}{N_{\text {rep }}-1} \sum_{k}^{N_{\text {rep }}} X_{l k} X_{k l^{\prime}}^{t}
$$

and therefore,

$$
\operatorname{cov}(Q)=\frac{1}{N_{\text {rep }}-1} X X^{t}
$$

A representation of the covariance matrix in terms of replicas is found by Singular Value Decomposition (SVD) of the matrix $X$. Namely, we can write $X$ as:

$$
X=U S V^{t}
$$

Assuming that $N_{\text {pdf }} N_{x}<N_{\text {rep }}, U$ is an orthogonal matrix of dimensions $N_{\text {pdf }} N_{x} \times N_{\text {rep }}$ which contains the orthogonal eigenvectors of the covariance matrix with nonzero eigenvalues; $S$ is a diagonal matrix of real positive elements, constructed out of the singular values of $X$, i.e., the square roots of the nonzero eigenvalues of the covariance matrix Eq. (12), multiplied by the normalization constant $\left(N_{\text {rep }}-1\right)^{\frac{1}{2}}$; and $V$ is an orthogonal $N_{\text {rep }} \times N_{\text {rep }}$ matrix of coefficients.

Because

$$
X X^{t}=U S^{2} U^{t}=(U S)(U S)^{t},
$$

the matrix $Z=U S$ has the property that

$$
Z Z^{t}=X X^{t}
$$

But also,

$$
Z=X V
$$

and thus $Z$ provides the sought-for representation of the covariance matrix as a linear combination of Monte Carlo replicas.

Note that by 'linear combination of replicas' here we mean that each eigenvector is a combination of the original PDF replicas $f_{\alpha}^{(k)}\left(x_{i}, Q\right)$, i.e., with coefficients which depend on the replica index $k$, but do not depend on either the PDF index $\alpha$ or the value of $x$; nor, because of linearity of QCD evolution, on the scale $Q$. The method therefore allows for combination of PDFs that evolve in slightly different ways, with results that do not depend on the scale at which the SVD is performed.

A reduction of the number of eigenvectors $N_{\text {eig }}$ can now be performed using Principal Component Analysis (PCA). Since we observe that in practice many of the eigenvectors lead to a very small contribution to the covariance matrix, we can select a smaller set of $N_{\text {eig }}<N_{\text {eig }}^{(0)}$ eigenvectors which still provides a good approximation to the covariance matrix by selecting the $N_{\text {eig }}$ eigenvectors with largest eigenvalues.

Denoting with $u, s$, and $v$ the $N_{\text {pdf }} N_{x} \times N_{\text {eig }}, N_{\text {eig }} \times N_{\text {eig }}$ and $N_{\text {eig }} \times N_{\text {rep }}$ reduced matrices computed using these eigenvalues, for a given value of $N_{\text {eig }}$, using $v$ instead of $V$ in Eq. (17) minimizes the difference between the original and reduced covariance matrix

$$
\Delta \equiv\left\|U S^{2} U^{t}-u s^{2} u^{t}\right\|
$$

This method is guaranteed by construction to reproduce the covariance matrix of the initial prior set, that is, both PDF uncertainties and PDF correlations with an arbitrary level of 
precision, upon including more eigenvectors. It is also numerically stable (in the sense that the SVD yields well conditioned matrices), and the same methodology can be applied no matter which input PDF sets are used in the Monte Carlo combination.

In practice, we add eigenvectors until the size of the differences between the new Hessian and the original MC representations becomes comparable to the accuracy of the Gaussian approximation in that specific region. This can be characterized by the difference between the $1 \sigma$ and $68 \%$ CL intervals of the distribution of results when computing LHC cross-sections prior Monte Carlo set MC900. The $1 \sigma$ interval will overestimate the effect of the outlier replicas in comparison with the $68 \%$ CL intervals. Note that insisting that the method reproduces the $1 \sigma$ band exactly would actually lead to bigger differences between the prior and the Hessian distribution, as measured for example by the Kolmogorov distance between both. Therefore we add eigenvectors until the Hessian prediction for the $1 \sigma$ ends up between the $68 \%$ CL and the $1 \sigma$ intervals of the prior MC900.

As will be discussed in the comparison section below, we find that in the mc2hessian algorithm provides a satisfactory description of both the data regions and the extrapolation regions can be realized with $N_{\text {eig }}=100$ symmetric eigenvectors, for all relevant of observables. Decreasing this number does not seem advisable, since then one needs to provide $a d-h o c$ assumptions to increase the correlation lengths of the points in the small and large- $x$ regions.

\subsubsection{Choice of Hessian sets}

Extensive benchmarking of the two available Hessian methods has been performed, both at the level of parton distributions, luminosities, and LHC cross-sections. The complete set of comparison plots can be found at the PDF4LHC website:

http://www.hep.ucl.ac.uk/pdf4lhc/mc2h-gallery/website

It turns out that for a low number of eigenvectors, of the order of $N_{\text {eig }} \simeq 30$, META-PDFs has a somewhat superior performance, leading to a generally satisfactory representation of the PDF covariance matrix except in outlying regions, where the META-PDF uncertainties are smaller than those of the MC900 prior because of its minimal nature (cf. Sect. 5.3.1). On the other hand, for a larger number of eigenvectors, $N_{\text {eig }} \simeq 100$, the MCH-PDFs yield an extremely accurate representation of the covariance matrix with a somewhat improved performance as compared to the META-PDF method. For instance, as will be shown below, the entries of the PDF correlation matrix can be reproduced at the per-mile level.

For these reasons, in the following the Hessian PDF4LHC15 combined sets with $N_{\text {eig }}=30$ eigenvectors

PDF4LHC15_nlo_30, PDF4LHC15_nnlo_30.

are constructed from the META30 sets, while those with $N_{\text {eig }}=100$ eigenvectors

PDF4LHC15_nlo_100

PDF4LHC15_nnlo_100

are constructed from the MCH100 sets.

The MP4LHC code used to generate the META sets is publicly available from the HepForge website 
https://metapdf.hepforge.org/.

The mc2Hessian code use to generate the $\mathrm{MC} 2 \mathrm{H}$ sets is also publicly available from:

https://github.com/scarrazza/mc2Hessian.

together with the corresponding user documentation. For completeness, Sect. 6.2 reports the formulae that should be used to compute the $\mathrm{PDF}$ and $\mathrm{PDF}+\alpha_{s}$ uncertainties whenever any of the two Hessian sets, PDF4LHC15_100 and PDF4LHC15_30, are used.

\subsection{Comparisons and benchmarking}

Now we compare the three combined PDF sets resulting from applying different reduction strategies to the MC900 prior: the Monte Carlo set PDF4LHC15 mc with $N_{\text {rep }}=100$ replicas, and the two Hessian sets PDF4LHC15_100 and PDF4LHC15_30, with $N_{\text {eig }}=100$ and 30 symmetric eigenvectors respectively. We will only show here some representative comparison plots between the three NNLO sets, with the complete set of plots of PDFs, luminosities and LHC crosssections (as well as the corresponding comparisons at NLO) are available from the PDF4LHC website

\section{http://www.hep.ucl.ac.uk/pdf4lhc/mc2h-gallery/website}

We start the comparisons of the prior with the various reduced sets at the level of PDFs, which we perform at a representative LHC scale of $Q^{2}=10^{2} \mathrm{GeV}^{2}$. In Fig. 12 we compare the prior and the MC compressed set PDF4LHC15_nnlo_mc. We show results for the gluon, total quark singlet, anti-up quark and the isoscalar triplet, normalized to the central value of PDF4LHC15_nnlo_prior. As can be seen, there is good agreement in all the range in $x$ both for central values and for PDF uncertainties.

The corresponding comparison between the prior MC900 and the two reduced Hessian sets, PDF4LHC15_nnlo_100 and PDF4LHC15_nnlo_30, is shown in Fig. 13. In the case of the set with $N_{\text {eig }}=100$ eigenvectors, the agreement is good for all PDF combinations in the complete range of $x$. For the case of the set with $N_{\text {eig }}=30$ eigenvectors, the agreement is also good in the PDF $x$ range corresponding to precision physics measurements, but renders a slightly smaller uncertainty in the extrapolation regions at small- and large- $x$. Also for PDF combinations that are not known very well, like the isoscalar triplet, again the uncertainty of $N_{\text {eig }}=30$ sets can be below that of the prior in the poorly constrained regions.

Now we turn to a comparison of parton luminosities. In Fig. 14 we compare the parton luminosities at the LHC $13 \mathrm{TeV}$ computed with the prior set PDF4LHC15_nnlo_prior, with $N_{\text {rep }}=900$, and its compressed Monte Carlo representation, PDF4LHC15_nnlo_mc, for $\alpha_{s}\left(m_{Z}^{2}\right)=$ 0.118. We show the $g g, q g, q q$ and $q \bar{q}$ luminosities as a function of the invariant mass of the final state $M_{X}$, normalized to the central value of PDF4LHC15_nnlo_prior. We see reasonable agreement in all cases.

The corresponding comparison of PDF luminosities in the case of the reduced Hessian sets is shown in Fig. 15, where we compare the prior set PDF4LHC15_nnlo_prior and the two Hessian sets, PDF4LHC15_nnlo_100 and PDF4LHC15_nnlo_30. Both the $N_{\text {eig }}=100$ set and the $N_{\text {eig }}=30$ provide a good representation of the 900 replica prior in the region of $M_{X}$ relevant for precision physics while the the $N_{\text {eig }}=30$ performs slightly worse in the low mass region, sensitive to the small- $x$ PDFs. 

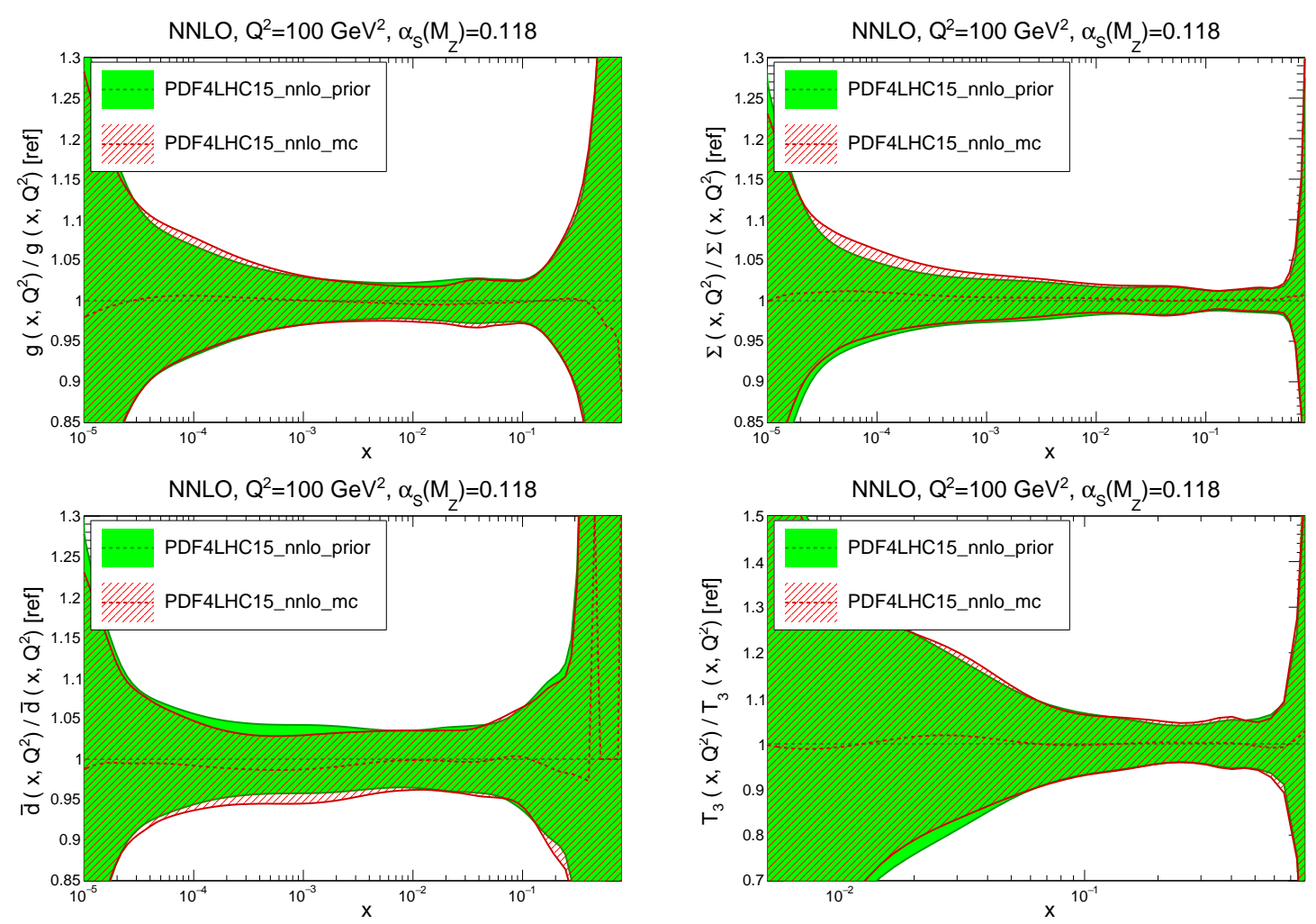

Figure 12: Comparison of PDFs at a scale $Q^{2}=10^{2} \mathrm{GeV}^{2}$ from the starting prior 900 replica set PDF4LHC15_nnlo_prior and the MC compressed set PDF4LHC15_nnlo_mc. We show results for the gluon, total quark singlet, anti-up quark and isoscalar triplet, normalized to the central value of PDF4LHC15_nnlo_prior.

A more detailed quantification of the differences in the three reduction methods can be obtained by computing the percentage difference of variances between the prior and its various reduced representations. Recall that in the Hessian approach, central values are reproduced automatically, as is the deviation from the central set which is parametrized by the error sets; in the Monte Carlo approach in principle, there can be a small shift in the mean when reducing the sample size, though this is of the order of the standard deviation of the mean (i.e. 10 time smaller than the standard deviation, for a 100-replica sample), further reduced by the compression method. We have explicitly checked that indeed in all cases the accuracy with which central values are reproduced is that of the LHAPDF6 interpolation with which final grids are delivered.

Results for the percentage differences in the variance (that is, in the PDF uncertainties) between the prior and each of the three reduced sets are shown in Fig. 16. We see that Hessian set with 100 eigenvectors reproduces in all cases the variances of the prior with precision at worst of $1 \%$, typically much better than that. The Monte Carlo set and the Hessian with 30 eigenvectors lead to similar performances in terms of reproducing the variances of the prior, with differences that can be up to $10 \%$.

We then turn to a comparison of PDF correlations. In Fig. 17 we show the difference between the PDF correlation coefficients computed with the prior set PDF4LHC15_nnlo_prior 

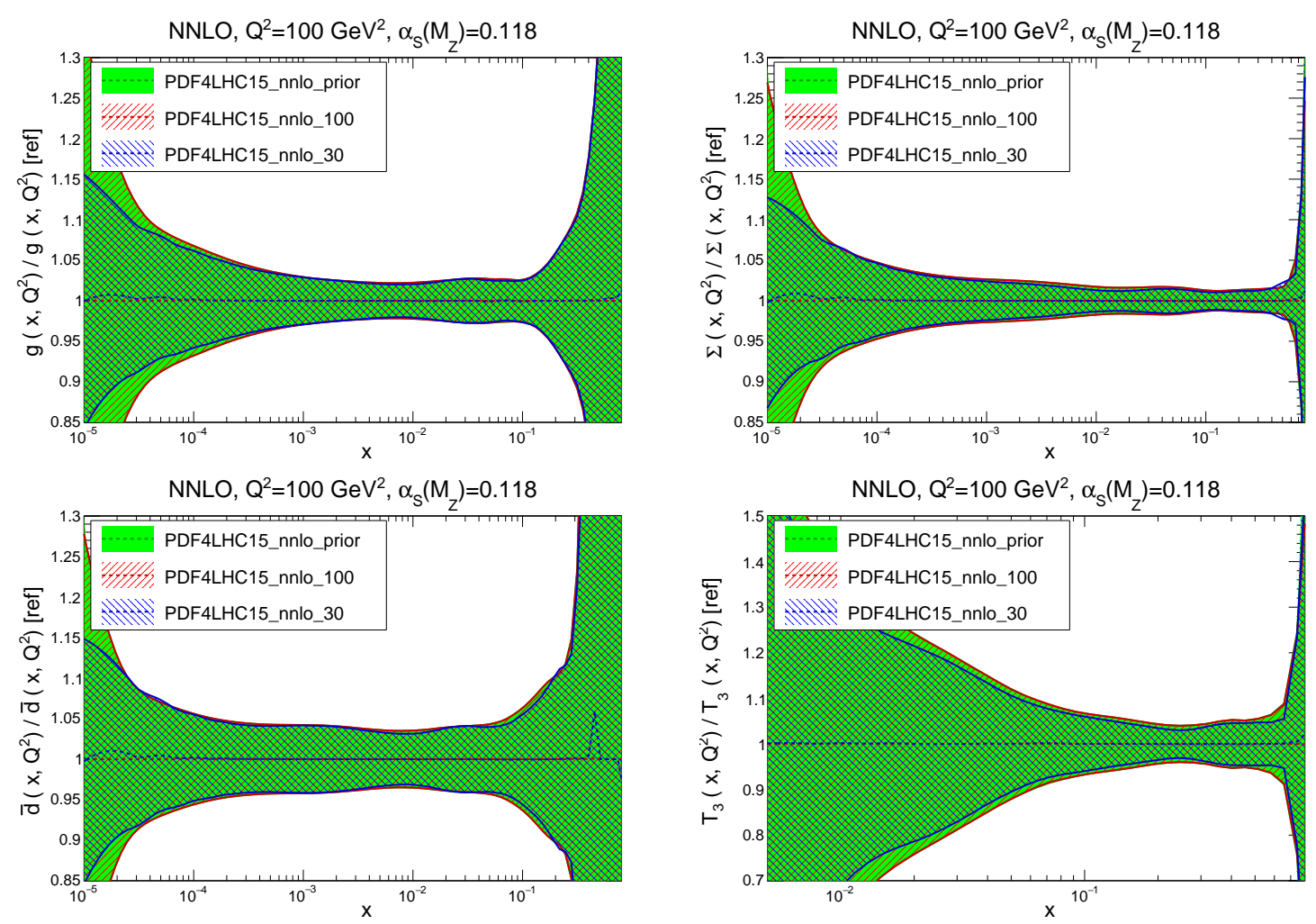

Figure 13: Same as Fig. 12 but now comparing the prior to the two Hessian sets, PDF4LHC15_nnlo_100 and PDF4LHC15_nnlo_30.

and its compressed Monte Carlo representation, PDF4LHC15_nnlo_mc. The plot shows the $7 \times 7$ matrix of all possible correlations of light flavor PDFs (three quarks and antiquarks and the gluon), sampled on logarithmically spaced $x$ values between $x_{\min }=10^{-5}$ and $x_{\max }=0.9$, and with $Q=8 \mathrm{GeV}$. In the left plot of Fig. 17 the range of difference shown is between -1 and 1 , while in the right plot we zoom in to a range of difference between -0.2 and 0.2 . We find good agreement in general, with differences never exceeding 0.2 in modulus. Note that the correlation coefficients can easily vary by $0.1-0.2$ or more among the input PDF sets.

The corresponding comparison of PDF correlations for the two Hessian sets, PDF4LHC15_nnlo_100 (upper plots) and PDF4LHC15_nnlo_30 (lower plots), is shown in Fig. 18. For the case of the $N_{\text {eig }}=100$ set the correlations are essentially identical to those of the prior, with differences below 0.01 . For the $N_{\text {eig }}=30$ set instead, typical differences in the correlation coefficients are somewhat larger, comparable to those found when using the MC set PDF4LHC15_nnlo_mc in Fig. 17 .

\subsection{Implications for LHC processes}

We now consider the comparison at the level of LHC cross-sections and distributions. All comparisons reported in this document have been performed using NLO matrix elements, for which fast computational tools are available, even when using NNLO PDFs: the comparison is performed for PDF validation purposes only. The NLO calculations that have been used for the validation presented in this report have been produced used APPLgrid [90] interfaced to 

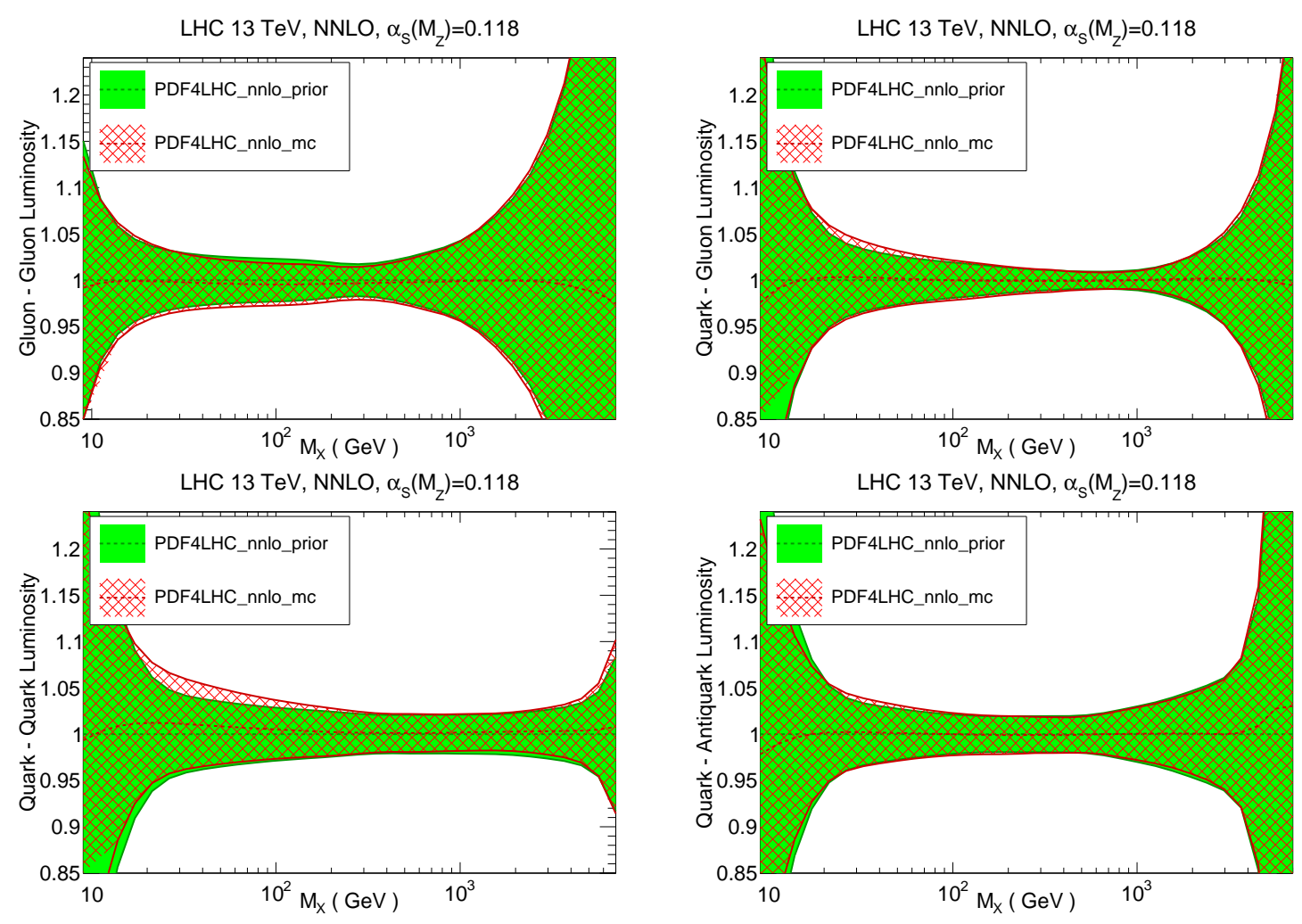

Figure 14: Comparison of parton luminosities at the LHC $13 \mathrm{TeV}$ computed using the prior set PDF4LHC15_nnlo_prior with $N_{\text {rep }}=900$, and its compressed Monte Carlo representation, PDF4LHC15_nnlo_mc, for $\alpha_{s}\left(m_{Z}^{2}\right)=0.118$. We show the $g g, q g, q q$ and $q \bar{q}$ luminosities as a function of the invariant mass of the final state $M_{X}$, normalized to the central value of PDF4LHC15_nnlo_prior.

NLOjet++ [89 and MCFM [15], and with aMCfast 117] interfaced to Madgraph5_aMC@NLO [118]. In all cases we use the default theory settings, including the scale choices, of the respective codes. Theory calculations have been performed at $7 \mathrm{TeV}$ for these processes for which data is already available and that have been used for PDF fits, see for example [11]; in addition, a number of dedicated grids for $13 \mathrm{TeV}$ processes have also been generated. In the former case the binning follows that to the corresponding experimental measurements, which we indicate in the list below.

In this report we will only show a representative subset of processes. The complete list of processes and kinematic distributions (available from the PDF4LHC15 webpage) is described now. At $7 \mathrm{TeV}$, the processes where experimental LHC measurements are available, and for which APPLgrid grids matching the experimental binning have been produced, are the following:

- Drell-Yan rapidity distributions in the LHCb forward region 36, 119.

- Invariant mass distribution from high-mass Drell-Yan (DY) [120].

- Rapidity distributions of $W$ and $Z$ production 34,50 .

- $p_{T}$ distribution of inclusive $W$ production 121 . 

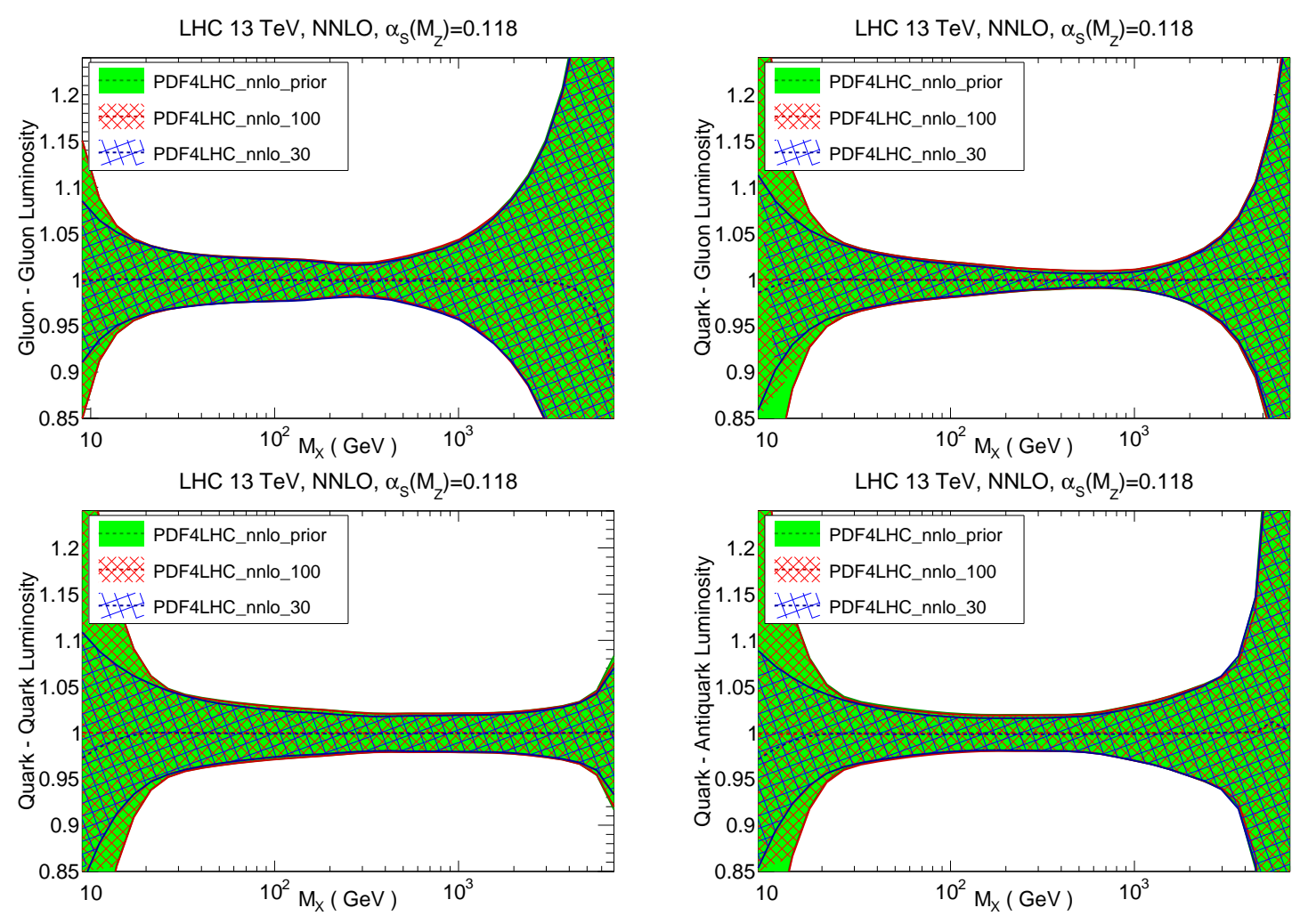

Figure 15: Same as Fig. 14 now comparing the parton luminosities from the prior set PDF4LHC15_nnlo_prior and the two Hessian sets, PDF4LHC15_nnlo_100 and PDF4LHC15_nnlo_30.

- Double differential DY distributions in dilepton mass and rapidity $[122$.

- Lepton rapidity distributions in $W+$ charm production [116].

- Inclusive jet production in the central and forward regions [33, 123].

In addition to these grids, at $13 \mathrm{TeV}$ we have generated specifically for this benchmark exercises a number of new fast NLO grids for differential distributions in Higgs, $t \bar{t}$ and vector boson production using Madgraph5_aMC@NLO interfaced to aMCfast, namely:

- Rapidity and $p_{T}$ distributions in inclusive $g g \rightarrow h$ production, as well as total crosssections for $h Z, h W$ and $h t \bar{t}$ production.

- Rapidity, $p_{T}$ and $m_{t \bar{t}}$ distributions in top-quark pair production.

- Missing $E_{T}$, lepton $p_{T}$ and rapidity, and transverse mass distributions in inclusive $W$ and $Z$ production.

First of all, let us go back to Figs. 10 and 11, which illustrated two cases of LHC crosssections where departures from the Gaussian regime were particularly striking. In these figures, the probability distributions computed from the MC900 prior, is compared to those computed using the CMC100 (PDF4LHC15_mc) and MCH100 (PDF4LHC15_100) reduced sets. It is clear 

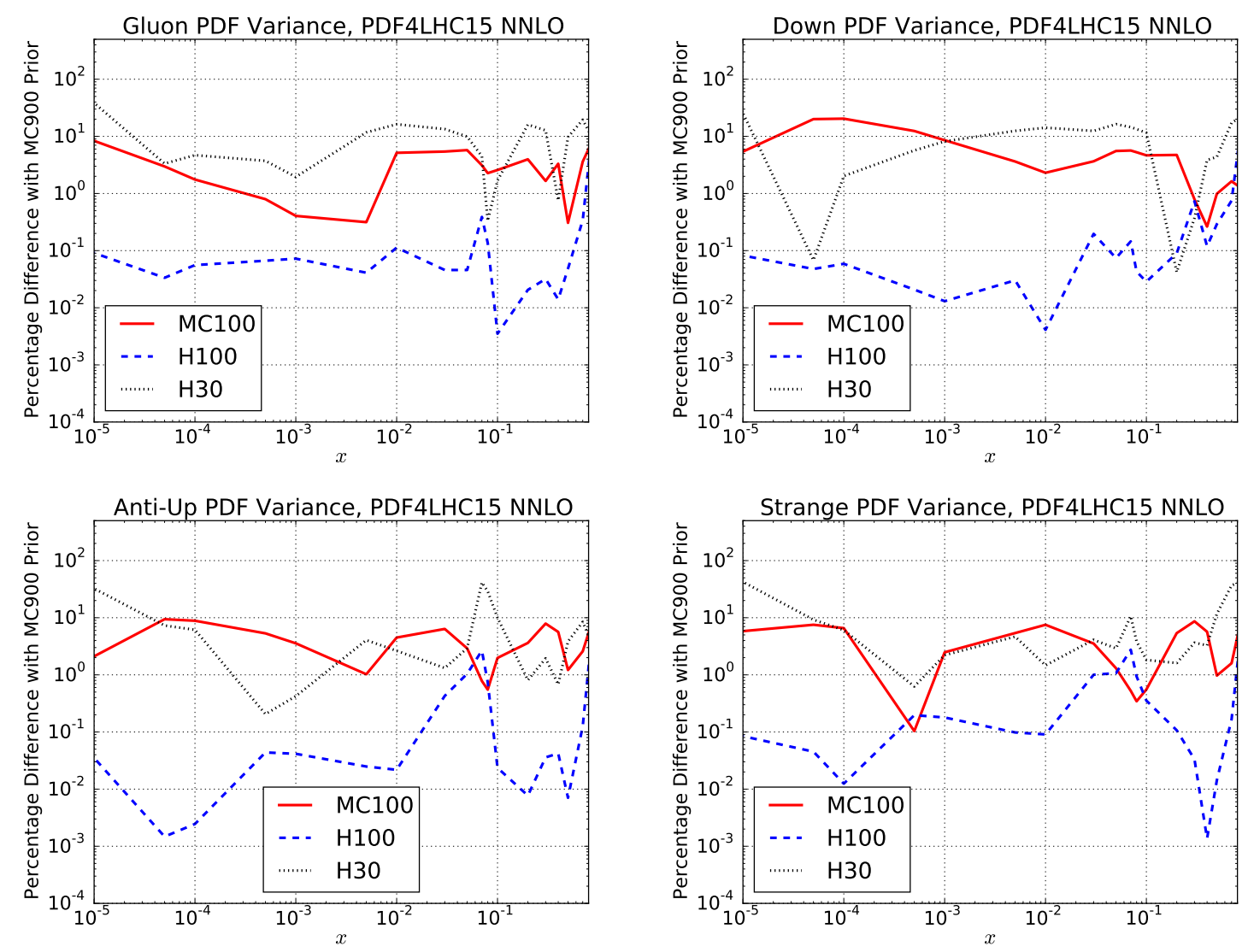

Figure 16: Percentage difference between the variances on the gluon, down, anti-up and strange distributions computed using the prior MC900 set, and each of the three reduced sets (see text).

that while the Monte Carlo PDF4LHC15_mc set is able to capture these non-Gaussian features, this is not the case for the Hessian set PDF4LHC15_100.

Then, in Fig. 19 we show the comparison between the three PDF4LHC15 combined sets for a representative selection of LHC cross-sections. In particular we show from top to bottom and from left to right the forward DY rapidity distributions at LHCb, the CMS DY doubledifferential distributions, the ATLAS 2010 inclusive jets in the central and forward regions (all these at $7 \mathrm{TeV}$ ) and then the $p_{T}$ and rapidity distributions of for Higgs production in gluon fusion, the $m_{t \bar{t}}$ distribution in $t \bar{t}$ production and the $Z p_{T}$ distribution (all these at $13 \mathrm{TeV}$ ). We compare the prior with the three reduced sets, the MC and the two Hessian sets. As can be seen, the agreement is in general good in all cases. 

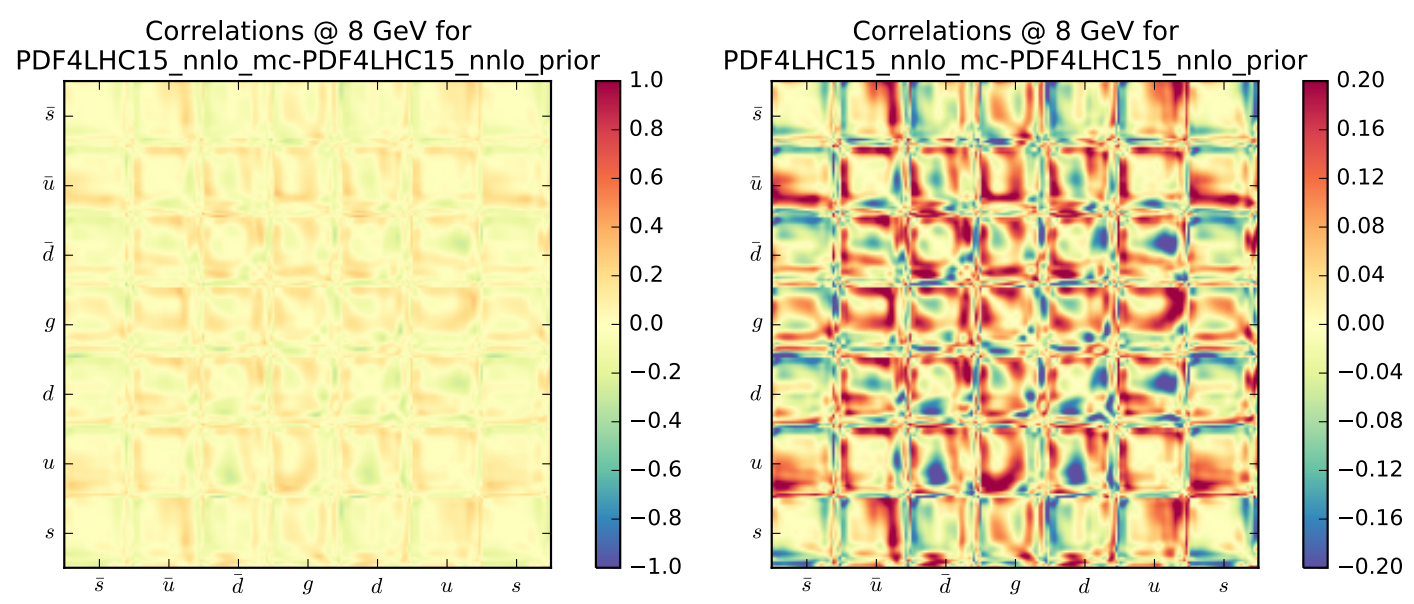

Figure 17: Left plot: difference between the PDF correlation coefficients computed with the prior PDF4LHC15_nnlo_prior and its compressed Monte Carlo representation, PDF4LHC15_nnlo_mc. The plot shows the $7 \times 7$ matrix for all possible comparisons of light flavor PDFs, with in each case $x$ ranging between $x_{\min }=10^{-5}$ and $x_{\max }=0.9$ (on a logarithmic scale) and fixed $Q=8 \mathrm{GeV}$. Right plot: same, but with the scale for the difference magnified to only cover the range from -0.2 to 0.2 .

An important application of the PDF4LHC15 combined sets is the computation of the correlation coefficients between LHC processes, such as for instance signal and background processes in Higgs production. Within the Higgs Cross Section Working Group (HXSWG), the correlation coefficients between some of these processes were computed using the original PDF4LHC recommendation: see Table 10 of Ref. [25]. We have recomputed these correlation coefficients using MC900, and compared the results with the three reduction methods. The processes included, at the $\mathrm{LHC} 13 \mathrm{TeV}$, are vector boson production, both $W$ and $Z, t \bar{t}$ production, and then Higgs production in gluon fusion $g g h$, in associated production $h Z$ and $h W$, and in association with a top quark pair, $h t \bar{t}$.

In Fig. 20 we show the difference between the correlation coefficients computed using the prior set PDF4LHC15_nnlo_prior and the two Hessian sets, PDF4LHC15_nnlo_100 (upper left plot) and PDF4LHC15_nnlo_30 (upper right plot) as well as with the MC set, PDF4LHC15_nnlo_mc (lower plot). As we can see, with the $N_{\text {eig }}=100$ set the correlation coefficients are always reproduced within a few percent, while for the $N_{\text {eig }}=30$ and the MC sets somewhat larger differences are found, a few instances up to 0.2 .

In addition, we have tabulated the correlation coefficients for various LHC total crosssections computed using the prior and the various reduced sets. First of all, in Table 1 we show the value of the correlation coefficient between the $Z$ production cross-sections and the $W, t \bar{t}$, $g g h, h t \bar{t}, h W$ and $h Z$ production cross-sections. We compare the PDF4LHC15 prior with the Monte Carlo and the two Hessian reduced sets, both at NLO and at NNLO.

The information from this table is the consistent with that shown in Fig. 20, namely the Hessian set with 100 eigenvectors reproduces always the correlation coefficient of the prior, with an accuracy better than 1\%. Using the smaller Hessian set and the Monte Carlo set the correlation coefficients are reproduced not as perfectly, but still sufficiently well for most phenomenological applications. It should be kept in mind that the correlations themselves from the prior set have sizable uncertainties, in the sense that the individual correlations from 

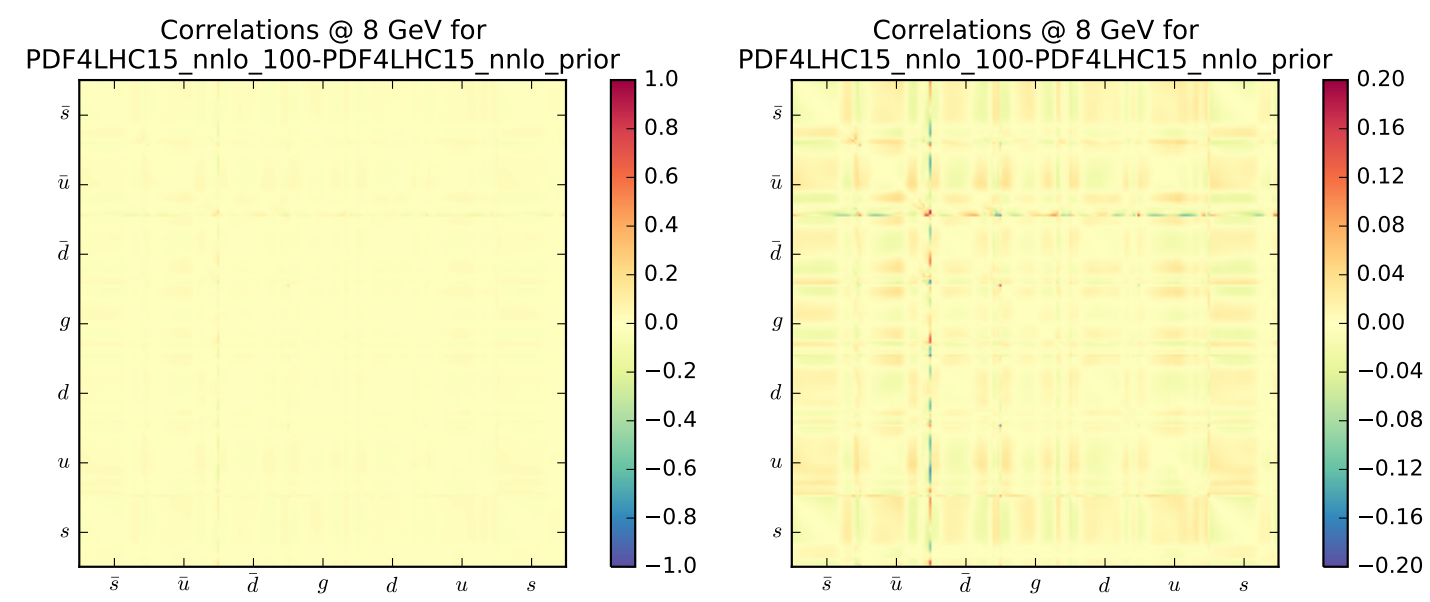

Correlations @ $8 \mathrm{GeV}$ for
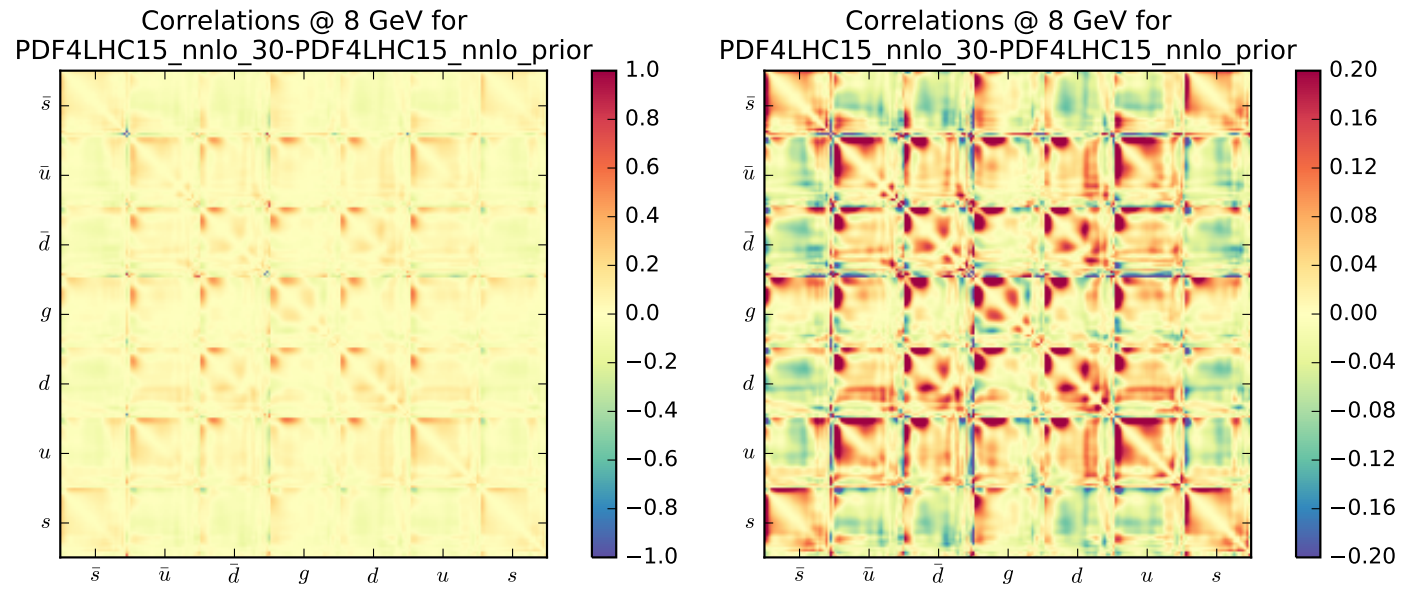

Figure 18: Same as Fig. 17 now comparing the the prior to the Hessian sets, PDF4LHC15_nnlo_100 (upper plots) and PDF4LHC15_nnlo_30 (lower plots).

the 3 different PDF families may differ significantly from each other. Thus, the correlations given in the tables provide an average behavior over the 3 PDF sets, and probably only the first digit in the correlation coefficient should be considered significant. The same conclusion can be derived from a variety of other pairs of LHC cross-sections, collected in Tables 2 to 4 . 

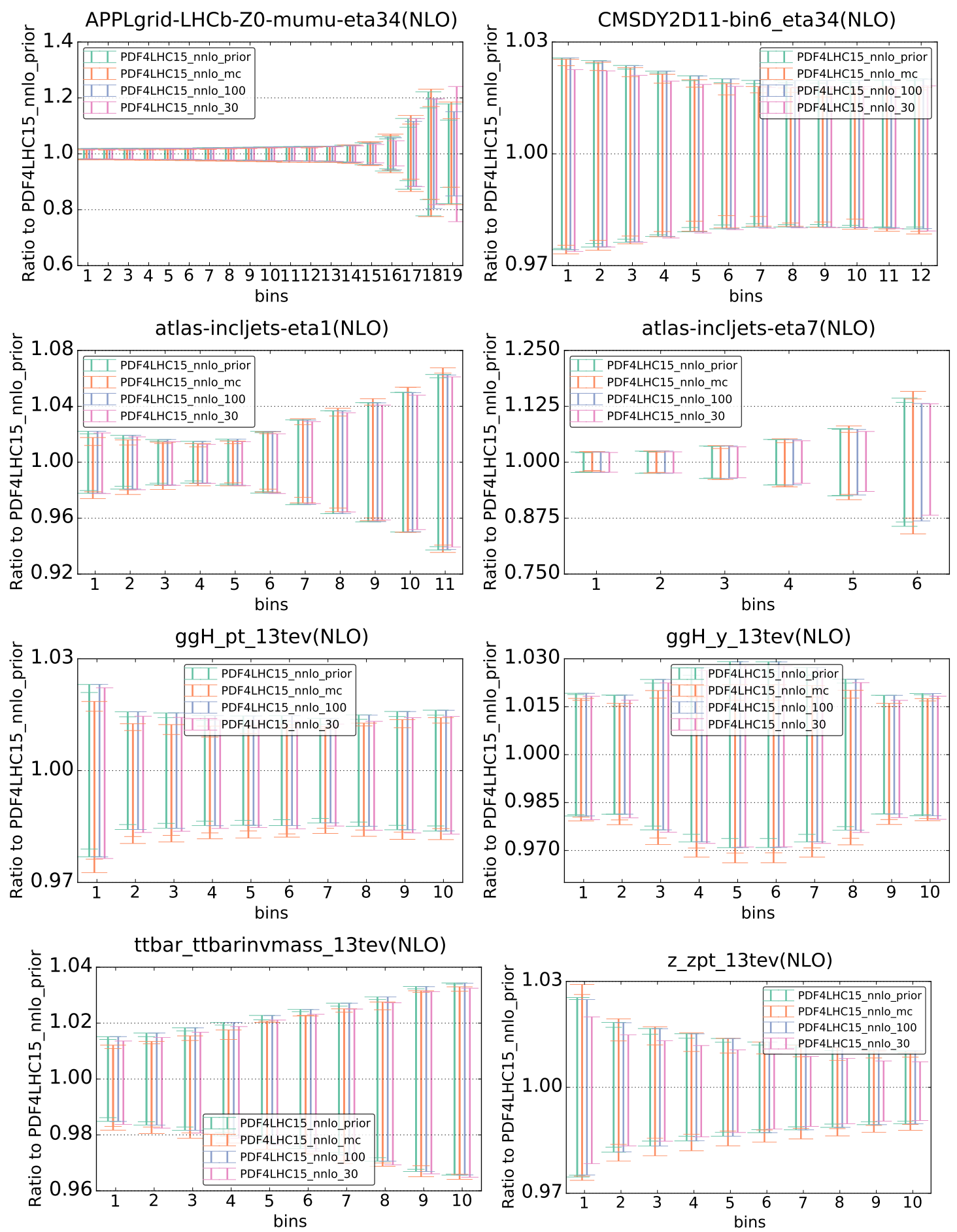

Figure 19: Comparison of differential distributions for several LHC processes, computed using the prior and the three reduced sets. From top to bottom and from left to right we show the forward DY rapidity distributions at LHCb, the CMS DY double-differential distributions, the ATLAS 2010 inclusive jets in the forward and central regions (all these at $7 \mathrm{TeV}$ ), the $p_{T}$ and rapidity distributions of $g g \rightarrow h$ production, the $m_{t \bar{t}}$ distribution in $t \bar{t}$ production and the $Z p_{T}$ distribution (all these at $13 \mathrm{TeV}$ ). See text for more details. 

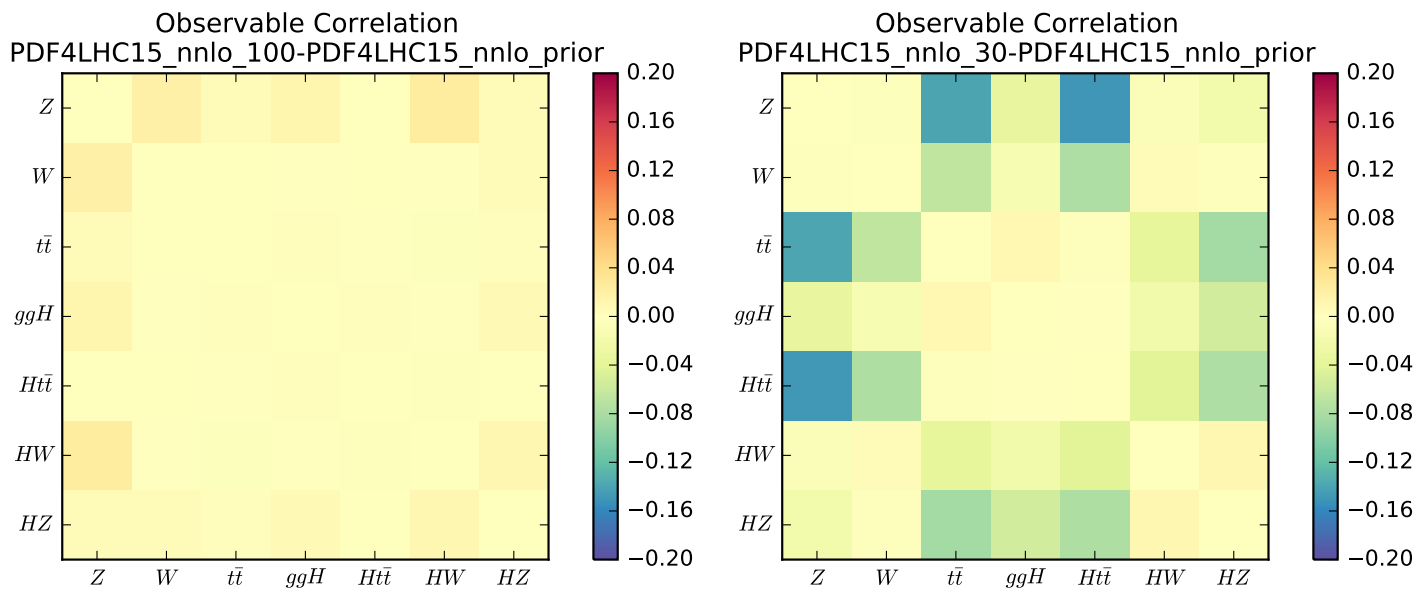

Observable Correlation PDF4LHC15_nnlo_mc-PDF4LHC15_nnlo_prior

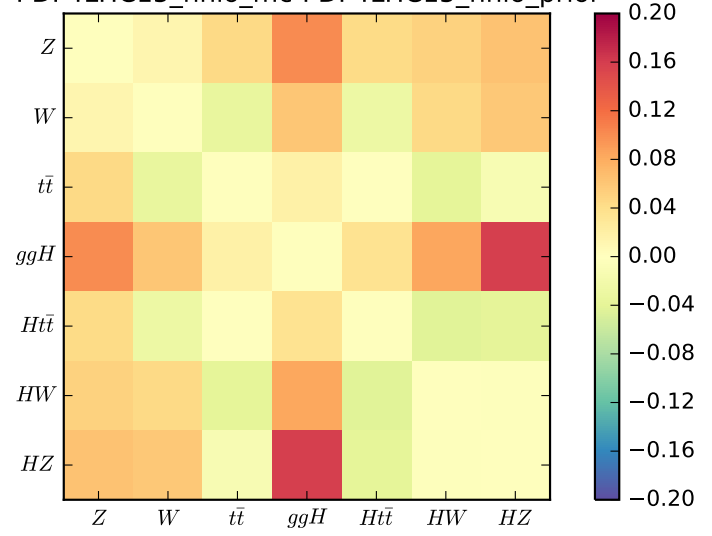

Figure 20: The difference between the correlation coefficients for a number of signal and background processes relevant for Higgs production at the LHC $13 \mathrm{TeV}$, see text for more details. We compare the correlation coefficients of the prior set PDF4LHC15_nnlo_prior with those of the two Hessian sets, PDF4LHC15_nnlo_100 (upper left plot) and PDF4LHC15_nnlo_30 (upper right plot), as well as with the PDF4LHC15_nnlo_mc set (lower plot).

\begin{tabular}{l|c|c|c|c|c|c}
\hline \multirow{2}{*}{ PDF Set } & \multicolumn{6}{|c}{ Correlation coefficient } \\
& $Z, W$ & $Z, t \bar{t}$ & $Z, g g h$ & $Z, h t \bar{t}$ & $Z, h W$ & $Z, h Z$ \\
\hline PDF4LHC15_nlo_prior & 0.90 & -0.60 & 0.22 & -0.64 & 0.55 & 0.74 \\
PDF4LHC15_nlo_mc & 0.92 & -0.49 & 0.41 & -0.58 & 0.61 & 0.77 \\
PDF4LHC15_nlo_100 & 0.92 & -0.60 & 0.23 & -0.64 & 0.57 & 0.75 \\
PDF4LHC15_nlo_30 & 0.90 & -0.68 & 0.16 & -0.71 & 0.55 & 0.76 \\
\hline \hline PDF4LHC15_nnlo_prior & 0.89 & -0.49 & 0.08 & -0.46 & 0.56 & 0.74 \\
PDF4LHC15_nnlo_mc & 0.90 & -0.44 & 0.18 & -0.42 & 0.62 & 0.80 \\
PDF4LHC15_nnlo_100 & 0.91 & -0.48 & 0.09 & -0.46 & 0.59 & 0.74 \\
PDF4LHC15_nnlo_30 & 0.88 & -0.63 & 0.04 & -0.61 & 0.56 & 0.72 \\
\hline
\end{tabular}

Table 1: Correlation coefficient between the $Z$ production cross-sections and the $W, t \bar{t}, g g h, h t \bar{t}, h W$ and $h Z$ production cross-sections. The PDF4LHC15 prior is compared to the Monte Carlo and the two Hessian reduced sets, both at NLO and at NNLO. 


\begin{tabular}{l|c|c|c|c|c|c}
\hline \multirow{2}{*}{ PDF Set } & \multicolumn{7}{|c}{ Correlation coefficient } \\
& $W, t \bar{t}$ & $W, g g h$ & $W, h t \bar{t}$ & $W, h W$ & $W, h Z$ & $t \bar{t}, g g h$ \\
\hline PDF4LHC15_nlo_prior & -0.46 & 0.32 & -0.51 & 0.77 & 0.78 & 0.27 \\
PDF4LHC15_nlo_mc & -0.35 & 0.49 & -0.46 & 0.81 & 0.80 & 0.27 \\
PDF4LHC15_nlo_100 & -0.47 & 0.32 & -0.52 & 0.77 & 0.79 & 0.27 \\
PDF4LHC15_nlo_30 & -0.52 & 0.28 & -0.56 & 0.79 & 0.81 & 0.32 \\
\hline \hline PDF4LHC15_nnlo_prior & -0.40 & 0.20 & -0.40 & 0.76 & 0.77 & 0.30 \\
PDF4LHC15_nnlo_mc & -0.44 & 0.26 & -0.42 & 0.81 & 0.82 & 0.32 \\
PDF4LHC15_nnlo_100 & -0.40 & 0.20 & -0.40 & 0.76 & 0.77 & 0.30 \\
PDF4LHC15_nnlo_30 & -0.47 & 0.19 & -0.47 & 0.77 & 0.76 & 0.31 \\
\hline
\end{tabular}

Table 2: Same as Table 1 for the correlation coefficient of additional pairs of LHC inclusive crosssections.

\begin{tabular}{l|c|c|c|c|c|c}
\hline \multirow{2}{*}{ PDF Set } & \multicolumn{7}{|c}{ Correlation coefficient } \\
& $t \bar{t}, H t \bar{t}$ & $t \bar{t}, h W$ & $t \bar{t}, h Z$ & $g g h, h t \bar{t}$ & $g g h, h W$ & $g g h, h Z$ \\
\hline PDF4LHC15_nlo_prior & 0.93 & -0.22 & -0.50 & -0.02 & 0.15 & 0.08 \\
PDF4LHC15_nlo_mc & 0.92 & -0.14 & -0.41 & -0.04 & 0.33 & 0.27 \\
PDF4LHC15_nlo_100 & 0.93 & -0.22 & -0.48 & -0.03 & 0.15 & 0.08 \\
PDF4LHC15_nlo_30 & 0.93 & -0.25 & -0.54 & 0.02 & 0.11 & -0.01 \\
\hline \hline PDF4LHC15_nnlo_prior & 0.87 & -0.23 & -0.34 & -0.13 & -0.01 & -0.17 \\
PDF4LHC15_nnlo_mc & 0.87 & -0.27 & -0.35 & -0.10 & 0.07 & -0.01 \\
PDF4LHC15_nnlo_100 & 0.87 & -0.24 & -0.34 & -0.13 & -0.02 & -0.17 \\
PDF4LHC15_nnlo_30 & 0.87 & -0.27 & -0.43 & -0.13 & -0.04 & -0.23 \\
\hline
\end{tabular}

Table 3: Same as Table 1 for the correlation coefficient of additional pairs of LHC inclusive crosssections.

\begin{tabular}{l|c|c|c}
\hline \multirow{2}{*}{ PDF Set } & \multicolumn{3}{|c}{ Correlation coefficient } \\
& $H t \bar{t}, H W$ & $H t \bar{t}, H Z$ & $H W, H Z$ \\
\hline PDF4LHC15_nlo_prior & -0.18 & -0.43 & 0.88 \\
PDF4LHC15_nlo_mc & -0.15 & -0.41 & 0.87 \\
PDF4LHC15_nlo_100 & -0.18 & -0.42 & 0.89 \\
PDF4LHC15_nlo_30 & -0.19 & -0.46 & 0.88 \\
\hline \hline PDF4LHC15_nnlo_prior & -0.13 & -0.17 & 0.90 \\
PDF4LHC15_nnlo_mc & -0.17 & -0.21 & 0.90 \\
PDF4LHC15_nnlo_100 & -0.13 & -0.17 & 0.91 \\
PDF4LHC15_nnlo_30 & -0.17 & -0.25 & 0.91 \\
\hline
\end{tabular}

Table 4: Same as Table 1 for the correlation coefficient of additional pairs of LHC inclusive crosssections. 


\begin{tabular}{|c|c|c|c|c|}
\hline LHAPDF6 grid & Pert order & ErrorType & $N_{\text {mem }}$ & $\alpha_{s}\left(m_{Z}^{2}\right)$ \\
\hline PDF4LHC15_nnlo_mc & NNLO & replicas & 100 & 0.118 \\
\hline PDF4LHC15_nnlo_100 & NNLO & symmhessian & 100 & 0.118 \\
\hline PDF4LHC15_nnlo_30 & NNLO & symmhessian & 30 & 0.118 \\
\hline PDF4LHC15_nnlo_mc_pdfas & NNLO & replicastas & 102 & $\begin{array}{l}\text { mem } 0: 100 \rightarrow 0.118 \\
\text { mem } 101 \rightarrow 0.1165 \\
\text { mem } 102 \rightarrow 0.1195\end{array}$ \\
\hline PDF4LHC15_nnlo_100_pdfas & NNLO & symmhessiantas & 102 & $\begin{array}{l}\text { mem } 0: 100 \rightarrow 0.118 \\
\text { mem } 101 \rightarrow 0.1165 \\
\text { mem } 102 \rightarrow 0.1195\end{array}$ \\
\hline PDF4LHC15_nnlo_30_pdfas & NNLO & symmhessiantas & 32 & $\begin{array}{l}\text { mem } 0: 30 \rightarrow 0.118 \\
\text { mem } 31 \rightarrow 0.1165 \\
\text { mem } 32 \rightarrow 0.1195\end{array}$ \\
\hline PDF4LHC15_nnlo_asvar & NNLO & - & 1 & $\begin{array}{l}\text { mem } 0 \rightarrow 0.1165 \\
\text { mem } 1 \rightarrow 0.1195\end{array}$ \\
\hline
\end{tabular}

Table 5: Summary of the combined NNLO PDF4LHC15 sets with $n_{f}^{\max }=5$ that are available from LHAPDF6. The corresponding NLO sets are also available. Members 0 and 1 of PDF4LHC15_nnlo_asvar coincide with members 101 and 102 (31 and 32) of PDF4LHC15_nnlo_mc_pdfas and PDF4LHC15_nnlo_100_pdfas (PDF4LHC15_nnlo_30_pdfas). Recall that in LHAPDF6 there is always a zeroth member, so that the total number of PDF members in a given set is always $N_{\text {mem }}+1$. See text for more details.

\section{The PDF4LHC 2015 recommendations}

The 2015 PDF4LHC prescription has been motivated, and its general principles spelled out, in Sect. 4. As discussed there, while in some cases individual PDF sets should always be used, for other LHC applications a combination of PDF sets is required. For these cases, in Sect. 5 we have constructed the statistical combination of the CT14, MMHT14 and NNPDF3.0 PDF sets using the Monte Carlo method. This strategy is different from the envelope method employed in the 2010 recommendations, and allows a robust statistical interpretation of the ensuing PDF uncertainties

The PDF4LHC15 combined sets are then delivered using three different options, corresponding in all cases to the same underlying prior combination. The choice of which delivery method should be used depends on purely practical considerations, such as whether a Hessian or Monte Carlo representation is preferred, or on the trade-off between computational speed and accuracy. Explicit recommendations for the usage of each of these three delivery options are provided below.

In this section we first present the final PDF4LHC15 sets which will be made available in LHAPDF6 and provide general guidelines for their usage. Then we review the formulae for the calculation of PDF and PDF $+\alpha_{s}$ uncertainties in each case. Finally, we present the PDF4LHC15 combined sets to be used in calculations in the $n_{f}=4$ scheme, and summarise the citation policy that should be used whenever these combined PDF sets are used.

We emphasize that the present document contains recommendations, not a series of unique instructions. We believe that with the delivery options provided here any PDF user will find the flexibility to choose the strategy that is better suited for each particular analysis. 


\subsection{Delivery and guidelines}

The PDF4LHC15 combined PDFs are based on an underlying Monte Carlo combination of CT14, MMHT14 and NNPDF3.0, denoted by MC900, which is made publicly available in three different reduced delivery forms:

- PDF4LHC15_mc: a Monte Carlo PDF set with $N_{\text {rep }}=100$ replicas.

- PDF4LHC15_30: a symmetric Hessian PDF set with $N_{\text {eig }}=30$ eigenvectors.

- PDF4LHC15_100: a symmetric Hessian PDF set with $N_{\text {eig }}=100$ eigenvectors.

In the three cases, combined sets are available at NLO and at NNLO, for the central value of $\alpha_{s}\left(m_{Z}^{2}\right)=0.118$. In addition, we provide additional sets which contain the central values for $\alpha_{s}\left(m_{Z}^{2}\right)=0.1165$ and $\alpha_{s}\left(m_{Z}^{2}\right)=0.1195$, and that can be used for the computation of the combined $\mathrm{PDF}+\alpha_{s}$ uncertainties, as explained in Sect. 6.2. Finally, for ease of usage, the combined sets for $\alpha_{s}\left(m_{Z}^{2}\right)=0.118$ are also presented bundled with the $\alpha_{s}$-varying sets in dedicated grid files. The specifications of each of the combined NNLO PDF4LHC15 sets that are available from LHAPDF6 are summarized in Table 5, note that the corresponding NLO sets are also available.

Usage of the PDF4LHC15 sets. As illustrated in Sect. 5, the three delivery options provide a reasonably accurate representation of the original prior combination. However, each of these methods has its own advantages and disadvantages, which make them more suited in different specific contexts. We now attempt to provide some general guidance about which of the three PDF4LHC15 combined sets should be used in specific phenomenological applications.

\section{Comparisons between data and theory for Standard Model measurements}

Recommendations: Use individual PDF sets, and, in particular, as many of the modern PDF sets [5 11] as possible.

Rationale: Measurements such as jet production, vector-boson single and pair production, or top-quark pair production, have the power to constrain PDFs, and this is best utilized and illustrated by comparing with many individual sets.

As a rule of thumb, any measurement that potentially can be included in PDF fits falls in this category.

The same recommendation applies to the extraction of precision SM parameters, such as the strong coupling $\alpha_{s}\left(m_{Z}^{2}\right)$ [75, 124], the $W$ mass $M_{W}$ [125], and the top quark mass $m_{t}[126]$ which are directly correlated to the PDFs used in the extraction.

\section{Searches for Beyond the Standard Model phenomena}

Recommendations: Use the PDF4LHC15_mc sets.

Rationale: BSM searches, in particular for new massive particles in the TeV scale, often require the knowledge of PDFs in regions where available experimental constraints are limited, notably close to the hadronic threshold where $x \rightarrow 1$ [127]. In these extreme kinematical regions the PDF uncertainties are large, the Monte Carlo combination of PDF sets is likely to be non-Gaussian. c.f. Figs. 10 and 11 . 
This case also applies to the calculations of PDF uncertainties in related theoretical analysis, such as when determining exclusion limits for specific BSM scenarios.

If it is necessary to use a Hessian representation of the PDF uncertainty, for example in order to express PDF errors as Gaussian systematic uncertainties, one can cross-check the PDF4LHC15_mc results with the two Hessian sets, PDF4LHC15_30 and PDF4LHC15_100, depending on the required accuracy.

3. Calculation of PDF uncertainties in situations when computational speed is needed, or a more limited number of error PDFs may be desirable

Recommendations: Use the PDF4LHC15_30 sets.

Rationale: In many situations, PDF uncertainties may affect the extraction of physics parameters. From the point of view of the statistical analysis, it might be useful in some cases to limit the number of error PDFs that need to be included in such analyses. In these cases, use of the PDF4LHC15_30 sets may be most suitable.

In addition, the calculation of acceptances, efficiencies or extrapolation factors are affected by the corresponding PDF uncertainty. These quantities are only a moderate correction to the measured cross-section, and thus a mild loss of accuracy in the determination of PDF uncertainties in these corrections is acceptable, while computational speed can be an issue. In these cases, use of the PDF4LHC15_30 sets is most suitable.

\section{Calculation of PDF uncertainties in precision observables}

Recommendation: Use the PDF4LHC15_100 sets.

Rationale: For several LHC phenomenological applications, the highest accuracy is sought for, with, in some cases, the need to control PDF uncertainties to the percent level, as currently allowed by the development of high-order computational techniques in the QCD and electroweak sectors of the Standard Model.

Whenever the highest accuracy is desired, the PDF4LHC15_100 set is most suitable.

However, calculations that have little impact on the PDF dependence of the precision measurement, such as certain acceptances, may also be computed according to (3), using the PDF4LHC15_30 set.

Concerning the specific applications of the four cases listed above, there are some important caveats to take into account.

- For the same process, more than one of the user cases above might be applicable. For instance, consider the total top quark production cross-section $\sigma(t \bar{t})$ : one should use either (3) or (4) to estimate the total PDF uncertainty (for instance to determine the overall compatibility of the SM theory and data for this measurement), and at the same time use (1) to gauge the sensitivity of this observable to constrain PDFs.

Therefore, cases (1)-(4) above are not exclusive: one or the other should be more adequate depending on the theoretical interpretation of a given experimental measurement.

- Since the three delivery methods are based on the same underlying PDF combination, it is perfectly consistent to use different delivery options in different parts of the same calculation. 
For instance, for the computation of the production cross-sections of high-mass BSM resonances, one could use the PDF4LHC15 mc sets to estimate the PDF uncertainty in the expected signal yield, and at the same time the PDF4LHC15_30 sets to estimate the PDF uncertainties that affect the corresponding acceptance calculation.

- In the case of a really significant discrepancy between the experimental data and theoretical calculations, it may be crucial to fully exclude the possibility that this discrepancy arises due to the PDFs.

In this case we recommend to compare data with a broader variety of PDFs, including PDF sets without LHC data, or PDF sets based only on DIS data.

\subsection{Formulae for the calculation of $\mathrm{PDF}$ and $\mathrm{PDF}+\alpha_{s}$ uncertainties}

For completeness, we also collect in this report the explicit formulae for the calculation of $\mathrm{PDF}$ and combined PDF $+\alpha_{s}$ uncertainties in LHC cross-sections when using the PDF4LHC15 combined sets. Let us assume that we wish to estimate the $\mathrm{PDF}+\alpha_{s}$ uncertainty of given crosssection $\sigma$, which could be a total inclusive cross-section or any bin of a differential distribution.

First of all, to compute the PDF uncertainty, one has to evaluate this cross-section $N_{\text {mem }}+1$ times, where $N_{\text {mem }}$ is the number of error sets (either symmetric eigenvectors or MC replicas) of the specific combined set,

$$
\sigma^{(k)}, \quad k=0, \ldots, N_{\text {mem }},
$$

so in particular $N_{\text {mem }}=30$ in PDF4LHC15_30 and $N_{\text {mem }}=100$ in PDF4LHC15_100 and PDF4LHC15_mc.

PDF uncertainties for Hessian sets. In the case of the Hessian sets, PDF4LHC15_30 and PDF4LHC15_100, the master formula to evaluate the PDF uncertainty is given by

$$
\delta^{\text {pdf }} \sigma=\sqrt{\sum_{k=1}^{N_{\text {mem }}}\left(\sigma^{(k)}-\sigma^{(0)}\right)^{2}},
$$

This uncertainty is to be understood as a $68 \%$ confidence level. From this expression it is also easy to determine the contribution of each eigenvector $k$ to the total Hessian PDF uncertainty.

PDF uncertainties for MC sets. For the case of the Monte Carlo sets, PDF4LHC15_mc, PDF uncertainties can be computed in two ways. First of all, one can use the standard deviation of the distribution

$$
\delta^{\text {pdf }} \sigma=\sqrt{\frac{1}{N_{\text {mem }}-1} \sum_{k=1}^{N_{\text {mem }}}\left(\sigma^{(k)}-\langle\sigma\rangle\right)^{2}},
$$

where the mean value of the cross-section, $\langle\sigma\rangle$, is computed as usual

$$
\langle\sigma\rangle=\frac{1}{N_{\mathrm{mem}}} \sum_{k=1}^{N_{\mathrm{mem}}} \sigma^{(k)} .
$$

For Monte Carlo sets one should use always Eq. 22 for the mean value of the cross-section, though in many cases it is true that $\langle\sigma\rangle \simeq \sigma^{(0)}$. 
Alternatively, PDF uncertainties in a Monte Carlo set can be computed from the $68 \%$ confidence level. This is achieved by reordering the $N_{\text {mem }}=100$ values for the cross-sections in ascending order, so that we have

$$
\sigma^{(1)} \leq \sigma^{(2)} \leq \cdots \leq \sigma^{\left(N_{\mathrm{mem}}-1\right)} \leq \sigma^{\left(N_{\mathrm{mem}}\right)},
$$

and then the PDF uncertainty computed as the $68 \%$ CL interval of will be given by

$$
\delta^{\text {pdf }} \sigma=\frac{\sigma^{(84)}-\sigma^{(16)}}{2} .
$$

This definition is suitable wherever departures from the Gaussian regime are sizable, since it gives the correct statistical weight to outliers. In general, it can be useful to compare results with the two expressions, Eq. (21) and Eq. (24), and whenever differences are found, use Eq. (24). In addition, in the non-Gaussian case the mean of the distribution Eq. $(22)$ is not necessarily the best choice for the central value of the cross-section, and we recommend to use instead the midpoint of the $68 \% \mathrm{CL}$ interval, that is

$$
\bar{\sigma}=\frac{\sigma^{(84)}+\sigma^{(16)}}{2} \text {. }
$$

Combined PDF $+\alpha_{s}$ uncertainties. Let us now turn to discuss the computation of the combined PDF $+\alpha_{s}$ uncertainties. The PDF4LHC15 combined are based on the following value of $\alpha_{s}\left(m_{Z}^{2}\right)$ and of its associated uncertainty,

$$
\alpha_{s}\left(m_{Z}^{2}\right)=0.1180 \pm 0.0015
$$

at the $68 \%$ confidence level, and both at NLO and at NNLO. This choice is consistent with the current PDG average [107, and reflects recent developments towards the updated 2015 $\mathrm{PDG}$ average ${ }^{4}$ It is then recommended that $\mathrm{PDF}+\alpha_{s}$ uncertainties are determined by first computing the PDF uncertainty for the central $\alpha_{s}$, using Eqs. (20), (21) or (24), then computing predictions for the upper and lower values of $\alpha_{s}$, consistently using the corresponding PDF sets, and finally adding results in quadrature.

Specifically, for the same cross-section $\sigma$ as before, the $\alpha_{s}$ uncertainty can be computed as:

$$
\delta^{\alpha_{s}} \sigma=\frac{\sigma\left(\alpha_{s}=0.1195\right)-\sigma\left(\alpha_{s}=0.1165\right)}{2},
$$

corresponding to an uncertainty $\delta \alpha_{s}=0.0015$ at the $68 \%$ confidence level. Note that Eq. 27) is to be computed with the central values of the corresponding PDF4LHC15 sets only. Needless to say, the same value of $\alpha_{s}\left(m_{Z}^{2}\right)$ should always be used in the partonic cross-sections and in the PDFs. The combined PDF $+\alpha_{s}$ uncertainty is then computed as follows

$$
\delta^{\mathrm{PDF}+\alpha_{\mathrm{s}}} \sigma=\sqrt{\left(\delta^{\mathrm{pdf}} \sigma\right)^{2}+\left(\delta^{\alpha_{s}} \sigma\right)^{2}} .
$$

The result for any other value of $\delta \alpha_{s}$, as compared to the baseline Eq. (26), can be obtained from a trivial rescaling of Eq. (27) assuming linear error propagation. That is, if we assume a different value for the uncertainty in $\alpha_{s}$,

$$
\tilde{\delta} \alpha_{s}=r \cdot \tilde{\delta} \alpha_{s}, \quad \delta \alpha_{s}=0.0015,
$$

\footnotetext{
${ }^{4}$ Preliminary results on the $2015 \mathrm{PDG}$ average for $\alpha_{s}\left(m_{Z}^{2}\right)$ have been presented in https://indico.cern. ch/event/392530/contribution/1/attachments/1168353/1686119/alphas-CERN2015.pdf
} 
then the combined PDF $+\alpha_{s}$ uncertainty Eq. 28 needs to be modified as follows

$$
\delta^{\mathrm{PDF}+\alpha_{\mathrm{s}}} \sigma=\sqrt{\left(\delta^{\mathrm{pdf}} \sigma\right)^{2}+\left(r \cdot \delta^{\alpha_{s}} \sigma\right)^{2}},
$$

everything else unchanged. It is thus clear from Eq. (30) that despite combined PDF sets are provided for a range of $\delta \alpha_{s}= \pm 0.0015$ around the central value, any other choice for $\delta \alpha_{s}$ can be trivially implemented using the existing PDF4LHC15 sets.

Implementation in LHAPDF6. Starting from LHAPDF v6.1.6, it is possible to automatically compute the combined $\mathrm{PDF}+\alpha_{s}\left(m_{Z}^{2}\right)$ uncertainties for the various cases listed above using the corresponding built-in routines ${ }^{5}$

PDF reweighting. Many NLO and NNLO matrix-element calculators and event generators allow the computation of PDF uncertainties without any additional CPU-time cost by means of PDF reweighting techniques. This functionality is for example available, among others, in MadGraph5_aMC@NLO [118, POWHEG [129], Sherpa [130], FEWZ [131 and RESBOS [132,133]. These $(N) N L O$ reweighting methods can be used in the same way for the PDF4LHC15 combined sets as for the individual sets.

In addition, an approximate $L O P D F$ reweighting is often used, where event weights are rescaled by ratios of PDFs, see for example Sect. 7 of the LHAPDF6 manual [115]. This LO PDF reweighting can be applied to the PDF4LHC15 combined sets, with the caveat that it is only approximately correct: (N)NLO PDF reweighting requires modifications of the LO reweighting formula. In particular, LO reweighting misses potentially large contributions from partonic channels that arise first at NLO.

Therefore, exact NLO and NNLO PDF reweighting should be used whenever possible, and the approximate LO PDF reweighting should be only used when the former is not available. The exception is of course LO event generators, where LO PDF reweighting is exact (except for the dependence of the PDF in the parton shower - but this is also true for NLO PDF reweighting).

\subsection{PDF4LHC15 combined sets in the $n_{f}=4$ scheme}

In addition to the combined sets listed in Table 5, which are suitable for calculations in the $n_{f}=5$ scheme, PDF4LHC15 combined sets have also been made available in the $n_{f}=4$ scheme. These are required for consistent calculations where the partonic cross-sections are computed in the $n_{f}=4$ scheme, accounting for bottom quark mass effects 134.

The inputs for the $n_{f}=4 \mathrm{PDF} 4 \mathrm{LHC} 15$ combination are the $n_{f}=4$ versions of the CT14, MMHT14 and NNPDF3.0 global fits. Each of these is constructed 6, 39, 135 from the corresponding $n_{f}=5$ global fits, using these as a boundary condition for $m_{c} \leq Q_{0} \leq m_{b}$, from which PDFs and $\alpha_{s}$ are obtained for $Q>Q_{0}$ using evolution equations with only four active quark flavours.

As it is well known, in the $n_{f}=4$ scheme, $\alpha_{s}\left(m_{Z}^{2}\right)$ is rather smaller than the global average value of 0.118 for $n_{f}=5$. For example, at NLO, using $n_{f}=4$ active flavours for the running of the strong couplings up to the $Z$ mass, one finds that $\alpha_{s}^{\left(n_{f}=5\right)}\left(m_{Z}^{2}\right)=0.118$ corresponds to $\alpha_{s}^{\left(n_{f}=4\right)}\left(m_{Z}^{2}\right) \simeq 0.113$. The exact value depends on the choice of $m_{b}$, which is slightly different

\footnotetext{
${ }^{5}$ We thank Graeme Watt for the implementation of the combined PDF $+\alpha_{s}$ uncertainties in LHAPDF6
} 
in each PDF group, though these differences are subdominant as compared to the PDF uncertainties in the combination. 6 When the exact matching conditions are unknown, $\alpha_{s}^{\left(n_{f}=4\right)}(\mu)$ at an arbitrary renormalization scale $\mu$ can also be determined from the corresponding $\alpha_{s}^{\left(n_{f}=5\right)}(\mu)$ using the scheme transformation relations of Ref. [137], currently known up to four loops in QCD.

\begin{tabular}{|c|c|c|c|c|}
\hline LHAPDF6 grid & Pert order & ErrorType & $N_{\text {mem }}$ & $\alpha_{s}^{\left(n_{f}=5\right)}\left(m_{Z}^{2}\right)$ \\
\hline PDF4LHC15_nlo_nf4_100 & $\mathrm{NLO}$ & symmhessian & 100 & 0.118 \\
\hline PDF4LHC15_nlo_nf4_30 & NLO & symmhessian & 30 & 0.118 \\
\hline PDF4LHC15_nlo_nf4_100_pdfas & NLO & symmhessiantas & 102 & $\begin{array}{l}\text { mem } 0: 100 \rightarrow 0.118 \\
\text { mem } 101 \rightarrow 0.1165 \\
\text { mem } 102 \rightarrow 0.1195\end{array}$ \\
\hline PDF4LHC15_nlo_nf4_30_p & NLO & symmhessiantas & 32 & $\begin{array}{l}\text { mem } 0: 30 \rightarrow 0.118 \\
\text { mem } 31 \rightarrow 0.1165 \\
\text { mem } 32 \rightarrow 0.1195\end{array}$ \\
\hline PDF4LHC15_nlo_nf4_asvar & NLO & - & 1 & $\begin{array}{l}\text { mem } 0 \rightarrow 0.1165 \\
\text { mem } 1 \rightarrow 0.1195\end{array}$ \\
\hline
\end{tabular}

Table 6: Same as Table 5 for the combined PDF4LHC15 sets in the $n_{f}=4$ scheme. We indicate the value of $\alpha_{s}^{\left(n_{f}=5\right)}\left(m_{Z}^{2}\right)$ in the $n_{f}=5$ scheme, the actual value in the $n_{f}=4$ scheme is substantially smaller, see text.

The available PDF4LHC15 combined sets in the $n_{f}=4$ scheme are summarised in Table 6 . Only the NLO combination is required, since no NNLO calculations in the $n_{f}=4$ scheme are yet available. Also, only Hessian sets are produced, since $n_{f}=4$ calculations are always in a region where the underlying PDF combination is essentially Gaussian.

\subsection{Citation policy for the PDF4LHC recommendation}

The techniques and methods presented in this report are the result of an intense collaborative efforts within the PDF4LHC community. The three reduction methods presented in Sect. 5 , the CMC-PDFs, META-PDFs and MC-H PDFs, have been substantially improved and refined (and in some cases, even developed from scratch) as a result of the fruitful discussions within the PDF4LHC working group. It is thus important to properly acknowledge this effort by providing an accurate citation policy for the usage of the PDF4LHC15 recommendations, which we spell out in some detail here:

- Whenever the PDF4LHC15 recommendations are used, this report should be cited.

- In addition, the individual PDF sets that enter the combination should also be cited:

1. CT14 [6]: S. Dulat, T. J. Hou, J. Gao, M. Guzzi, J. Huston, P. Nadolsky, J. Pumplin, C. Schmidt, D. Stump and C. P. Yuan, 'The CT14 Global Analysis of Quantum Chromodynamics', arXiv:1506.07443.

2. MMHT14 10]: L. A. Harland-Lang, A. D. Martin, P. Motylinski and R.S. Thorne, 'Parton distributions in the LHC era: MMHT 2014 PDFs', Eur. Phys. J. C75 (2015) 5, 204, arXiv:1412.3989.

\footnotetext{
${ }^{6}$ It is also possible to produce PDF sets in a hybrid scheme where $\alpha_{s}(Q)$ runs with five flavours, but where the DGLAP equations include only four active flavours, above the bottom threshold [135 136 .
} 
3. NNPDF3.0 [11]: R. D. Ball, V. Bertone, S. Carrazza, C. S. Deans, L. Del Debbio, S. Forte, A. Guffanti, N. P. Hartland, J. I. Latorre, J. Rojo and M. Ubiali, 'Parton Distributions for the LHC Run II', JHEP 1504 (2015) 040, arXiv:1410.8849.

In particular, citation of this report only without reference to the individual PDF sets that enter the combination is strongly discouraged.

- When any of the two Hessian sets are used, PDF4LHC15_30 or PDF4LHC15_100, the original publications where the Hessian reduction methods where developed should be cited:

1. META-PDFs [111]: J. Gao and P. Nadolsky, 'A meta-analysis of parton distribution functions', JHEP(1407)035, arXiv:1401.0013.

2. MCH-PDFs [112]: S. Carrazza, S. Forte, Z. Kassabov, J. I. Latorre and J. Rojo, 'An unbiased hessian representation for Monte Carlo PDFs', Eur. Phys. J. C 75, no. 8, 369 (2015), arXiv:1505.06736.

- When the Monte Carlo sets PDF4LHC15_mc are used, the original publication where the Monte Carlo compression method was developed should be cited:

1. CMC-PDFs 113]: S. Carrazza, J. I. Latorre, J. Rojo and G. Watt, 'A compression algorithm for the combination of PDF sets', Eur. Phys. J. C 75, no. 10, 474 (2015), arXiv:1504.06469.

- In addition, when either of the two reduction methods are employed, one should cite the original publication where the Monte Carlo representation of Hessian sets was presented 101]:

1. G. Watt and R. S. Thorne, 'Study of Monte Carlo approach to experimental uncertainty propagation with MSTW 2008 PDFs', JHEP 1208, 052 (2012), arXiv:1205.4024.

which is at the basis of the Monte Carlo method for the combination of PDF sets.

\section{$7 \quad$ Future directions}

We have presented in Sect. 4 the new PDF4LHC recommendation for the computation of $\mathrm{PDF}$ and combined $\mathrm{PDF}+\alpha_{s}$ uncertainties for LHC applications. While the general guideline remains to combine the individual PDFs when such combination is appropriate (cf. Sect. 4.1), both the way the combined uncertainties are determined, and their form of delivery, have evolved substantially since the last recommendation.

The main rationale for these changes is the observation that the PDFs of several groups have undergone significant evolution due to both new available data and methodological improvements, and that, as a consequence, there is now much better agreement between the PDF determinations based on the widest available dataset. The fact that the improvements are driven by increase of experimental information and theoretical understanding suggests that they are not accidental.

As a consequence, it now appears advisable to recommend a statistical combination of the PDFs from the three global analysis groups, with $\alpha_{s}$ uncertainties combined with PDF uncertainties in the standard way (albeit with a conservative estimate of the $\alpha_{s}$ uncertainty). 
In order to optimize and streamline usage of this statistical combination, we have produced a combined PDF4LHC15 set, both at NLO and NNLO. The set is delivered in three versions: as 100 Monte Carlo replicas, or as either 30 or 100 Hessian eigenvector sets. Guidelines for usage of the PDF4LHC15 set have been presented in Sect. 6, though it should be borne in mind that all its versions correspond to the same underlying information, and the choice of a specific version is motivated by practical considerations, such as speed versus accuracy.

Future updates of our current recommendation will require the release of new combined PDF sets, to replace the current PDF4LHC15 sets. In these future releases it might be desirable for all PDFs to use common values for heavy-quark masses, as it is currently done for the strong coupling $\alpha_{s}\left(m_{Z}^{2}\right)$. Such future updates are likely to be motivated by two sets of considerations.

First, we expect the PDF sets which enter the current combination to undergo various updates in the coming years, as a consequence of the availability of LHC Run-II data, and also, the availability of full NNLO corrections for a variety of processes that potentially provide important constraints on PDFs and that can be accurately measured at the LHC.

Second, while only the sets included in the current combination satisfy the requirements for inclusion in the combination spelled out in Sect. 4.1, this may change in the future. In particular, it may be possible to devise techniques for including non-global sets, using weighted statistical combination of PDF sets with uncertainties of different size or another method.

In the longer term, there are other important directions in the global PDF analysis that should be explored. Perhaps the most important one is a consistent estimation of theoretical uncertainties. While taking into account parametric uncertainties such as the value of $\alpha_{s}\left(m_{Z}^{2}\right)$ and the heavy quark masses is feasible with the current combination methods, estimating missing higher-order uncertainties is a more challenging problem, requiring investments by the individual PDF collaborations.

Another possible future avenue is to extend the PDF4LHC combinations beyond fixed-order QCD NLO and NNLO global fits, and to perform combinations of PDF sets with improved theory such as sets with QED corrections 66, 68, 69 (needed for consistent calculations when electroweak effects are included) or of sets with threshold resummation 138 (required when soft-gluon resummation is included in the partonic cross-sections). Eventually, one might even want to further improve the accuracy of global sets by using approximate N3LO calculations when available (for example for deep-inelastic coefficient functions [139, 140]).

To summarize, we have presented a general, robust, and statistically consistent procedure for combination of PDFs. It represents a significant advancement beyond the 2010 PDF4LHC recommendation [14], and aims to meet diverse needs of the LHC Run-II programme. The advancements described here bring the modern PDFs and their combinations to a novel level of accuracy, adequate for (N)NNLO QCD computations and analysis of vast experimental information anticipated in the near future.

\section{Acknowledgments}

We are grateful to Sergey Alekhin, Johannes Blümlein, Claire Gwenlan, Max Klein, Katerina Lipka, Kristin Lohwasser, Sven Moch, Klaus Rabbertz and Reisaburo Tanaka for their feedback on this report. We are also grateful to Richard Ball, André David, Lucian Harland-Lang, Maxime Gouzevitch, Jan Kretzschmar, José Ignacio Latorre, Alan Martin, Patrick Motylinski, Ringaile Placakyte, Jon Pumplin, Alessandro Tricoli, Dan Stump, Graeme Watt, C. P. Yuan, as well as to many other colleagues from the PDF4LHC Working Group community for 
illuminating discussions about the topics presented in this report.

S. C. and S. F. are supported in part by an Italian PRIN2010 grant and by a European Investment Bank EIBURS grant. S. F. and Z. K. are supported by the Executive Research Agency (REA) of the European Commission under the Grant Agreement PITN-GA-2012-316704 (HiggsTools). S.F. thanks Matteo Cacciari for hospitatly at LPTHE, Université Paris VI, where part of this work was done, supported by a Lagrange award. S. C. is also supported by the HICCUP ERC Consolidator grant (614577). The research of J. G. in the High Energy Physics Division at Argonne National Laboratory is supported by the U. S. Department of Energy, High Energy Physics, Office of Science, under Contract No. DE-AC02-06CH11357. The work of P. N. is supported by the U.S. Department of Energy under grant DE-SC0013681. J. R. is supported by an STFC Rutherford Fellowship and Grant ST/K005227/1 and ST/M003787/1, and by an European Research Council Starting Grant "PDF4BSM". The work of R. S. T. is supported partly by the London Centre for Terauniverse Studies (LCTS), using funding from the European Research Council via the Advanced Investigator Grant 267352. R. S. T. thanks the Science and Technology Facilities Council (STFC) for support via grant awards ST/J000515/1 and ST/L000377/1.

\section{References}

[1] J. Rojo et al., The PDF 4 LHC report on PDFs and LHC data: Results from Run I and preparation for Run II, J. Phys. G42 (2015) 103103, arXiv:1507.00556.

[2] S. Forte, Parton distributions at the dawn of the LHC, Acta Phys.Polon. B41 (2010) 2859, arXiv:1011.5247.

[3] S. Forte and G. Watt, Progress in the Determination of the Partonic Structure of the Proton, Ann.Rev.Nucl.Part.Sci. 63 (2013) 291, arXiv:1301.6754].

[4] C. Anastasiou, C. Duhr, F. Dulat, F. Herzog, and B. Mistlberger, Higgs Boson Gluon-Fusion Production in QCD at Three Loops, Phys. Rev. Lett. 114 (2015), no. 21 212001, arXiv:1503.06056.

[5] S. Alekhin, J. Blümlein, and S. Moch, The ABM parton distributions tuned to LHC data, Phys.Rev. D89 (2014) 054028, arXiv:1310.3059].

[6] S. Dulat et al., The CT14 Global Analysis of Quantum Chromodynamics, arXiv:1506.07443.

[7] J. F. Owens, A. Accardi, and W. Melnitchouk, Global parton distributions with nuclear and finite- $Q^{2}$ corrections, Phys. Rev. D87 (2013), no. 9 094012, arXiv:1212.1702.

[8] P. Jimenez-Delgado and E. Reya, Delineating parton distributions and the strong coupling, Phys.Rev. D89 (2014), no. 7 074049, [arXiv:1403.1852].

[9] H1 and ZEUS Collaboration, H. Abramowicz et al., Combination of Measurements of Inclusive Deep Inelastic $e^{ \pm} p$ Scattering Cross Sections and QCD Analysis of HERA Data, arXiv:1506.06042. 
[10] L. A. Harland-Lang, A. D. Martin, P. Motylinski, and R. S. Thorne, Parton distributions in the LHC era: MMHT 2014 PDFs, Eur. Phys. J. C75 (2015) 204, arXiv:1412.3989.

[11] NNPDF Collaboration, R. D. Ball et al., Parton distributions for the LHC Run II, JHEP 04 (2015) 040, arXiv:1410.8849.

[12] S. Alekhin et al., The PDF4LHC Working Group Interim Report, arXiv:1101.0536.

[13] R. D. Ball, S. Carrazza, L. Del Debbio, S. Forte, J. Gao, et al., Parton Distribution Benchmarking with LHC Data, JHEP 1304 (2013) 125, arXiv:1211.5142.

[14] M. Botje et al., The PDF4LHC Working Group Interim Recommendations, arXiv:1101.0538.

[15] J. Campbell and R. K. Ellis, Next-to-leading order corrections to $W+2 j e t$ and $Z+2 j e t$ production at hadron colliders, Phys. Rev. D65 (2002) 113007, hep-ph/0202176].

[16] S. Alekhin, J. Blümlein, S. Klein, and S. Moch, The 3-, 4-, and 5-flavor NNLO Parton from Deep-Inelastic- Scattering Data and at Hadron Colliders, Phys. Rev. D81 (2010) 014032, arXiv:0908.2766.

[17] P. M. Nadolsky et al., Implications of CTEQ global analysis for collider observables, Phys. Rev. D78 (2008) 013004, arXiv:0802.0007.

[18] H.-L. Lai et al., New parton distributions for collider physics, Phys. Rev. D82 (2010) 074024, arXiv: 1007.2241.

[19] M. Gluck, P. Jimenez-Delgado, and E. Reya, Dynamical parton distributions of the nucleon and very small-x physics, Eur.Phys.J. C53 (2008) 355, arXiv:0709.0614.

[20] M. Gluck, P. Jimenez-Delgado, E. Reya, and C. Schuck, On the role of heavy flavor parton distributions at high energy colliders, Phys. Lett. B664 (2008) 133, arXiv:0801.3618.

[21] H1 and ZEUS Collaboration, F. Aaron et al., Combined Measurement and QCD Analysis of the Inclusive $e^{ \pm} p$ Scattering Cross Sections at HERA, JHEP 1001 (2010) 109, arXiv:0911.0884.

[22] A. D. Martin, W. J. Stirling, R. S. Thorne, and G. Watt, Parton distributions for the LHC, Eur. Phys. J. C63 (2009) 189, arXiv:0901.0002.

[23] The NNPDF Collaboration, R. D. Ball et al., A first unbiased global NLO determination of parton distributions and their uncertainties, Nucl. Phys. B838 (2010) 136, arXiv:1002.4407.

[24] LHC Higgs Cross Section Working Group, S. Dittmaier, et al., Handbook of LHC Higgs Cross Sections: 1. Inclusive Observables, arXiv:1101.0593.

[25] LHC Higgs Cross Section Working Group, S. Dittmaier, et al., Handbook of LHC Higgs Cross Sections: 2. Differential Distributions, CERN-2012-002 (CERN, Geneva, 2012) arXiv:1201.3084. 
[26] F. Demartin, S. Forte, E. Mariani, J. Rojo, and A. Vicini, The impact of PDF and $\alpha_{s}$ uncertainties on Higgs Production in gluon fusion at hadron colliders, Phys. Rev. D82 (2010) 014002, arXiv:1004.0962.

[27] H.-L. Lai et al., Uncertainty induced by QCD coupling in the CTEQ global analysis of parton distributions, Phys. Rev. D82 (2010) 054021, arXiv:1004.4624.

[28] A. D. Martin, W. J. Stirling, R. S. Thorne, and G. Watt, Uncertainties on $\alpha_{S}$ in global PDF analyses, Eur. Phys. J. C64 (2009) 653-680, arXiv:0905.3531.

[29] J. Gao et al., CT10 next-to-next-to-leading order global analysis of QCD, Phys.Rev. D89 (2014) 033009, arXiv: 1302.6246.

[30] R. D. Ball et al., Parton distributions with LHC data, Nucl.Phys. B867 (2013) 244, arXiv:1207.1303.

[31] H1 and ZEUS Collaboration, A. Cooper-Sarkar, PDF Fits at HERA, PoS EPS-HEP2011 (2011) 320, arXiv:1112.2107].

[32] S. Alekhin, J. Blümlein, and S. Moch, Parton Distribution Functions and Benchmark Cross Sections at NNLO, Phys.Rev. D86 (2012) 054009, arXiv:1202.2281.

[33] ATLAS Collaboration, G. Aad et al., Measurement of inclusive jet and dijet production in pp collisions at $\sqrt{s}=7$ TeV using the ATLAS detector, Phys. Rev. D86 (2012) 014022, arXiv:1112.6297.

[34] ATLAS Collaboration, G. Aad et al., Measurement of the inclusive $W^{ \pm}$and $Z / \gamma^{*}$ cross sections in the electron and muon decay channels in pp collisions at $\sqrt{s}=7$ TeV with the ATLAS detector, Phys.Rev. D85 (2012) 072004, arXiv:1109.5141.

[35] CMS Collaboration, S. Chatrchyan et al., Measurement of the electron charge asymmetry in inclusive $W$ production in pp collisions at $\sqrt{s}=7$ TeV, Phys.Rev.Lett. 109 (2012) 111806, arXiv:1206.2598.

[36] LHCb Collaboration, R. Aaij et al., Inclusive $W$ and $Z$ production in the forward region at $\sqrt{s}=7$ TeV, JHEP 1206 (2012) 058, arXiv:1204.1620.

[37] J. Particle Data Group, Beringer et al., Review of Particle Physics (RPP), Phys.Rev. D86 (2012) 010001.

[38] PDF4LHC Steering Committee, "PDF4LHC Recommendations." http://www.hep.ucl.ac.uk/pdf4lhc/PDF4LHCrecom.pdf.

[39] The NNPDF Collaboration, R. D. Ball et al., Impact of Heavy Quark Masses on Parton Distributions and LHC Phenomenology, Nucl. Phys. B849 (2011) 296, arXiv:1101.1300.

[40] S. Forte, E. Laenen, P. Nason, and J. Rojo, Heavy quarks in deep-inelastic scattering, Nucl. Phys. B834 (2010) 116-162, [arXiv:1001.2312]. 
[41] The NNPDF Collaboration, R. D. Ball et al., Unbiased global determination of parton distributions and their uncertainties at NNLO and at LO, Nucl.Phys. B855 (2012) 153, arXiv:1107.2652.

[42] M. Guzzi, P. M. Nadolsky, H.-L. Lai, and C.-P. Yuan, General-Mass Treatment for Deep Inelastic Scattering at Two-Loop Accuracy, Phys.Rev. D86 (2012) 053005, arXiv:1108.5112.

[43] A. Martin, A. T. Mathijssen, W. Stirling, R. Thorne, B. Watt, et al., Extended Parameterisations for MSTW PDFs and their effect on Lepton Charge Asymmetry from W Decays, Eur.Phys.J. C73 (2013), no. 2 2318, arXiv:1211.1215.

[44] P. Jimenez-Delgado and E. Reya, Dynamical NNLO parton distributions, Phys.Rev. D79 (2009) 074023, arXiv:0810.4274.

[45] S. Alekhin, J. Blümlein, and S. Moch, Statistical issues in the parton distribution analysis of the Tevatron jet data, JHEP 02 (2014) 041, arXiv:1211.2642].

[46] Do Collaboration, V. M. Abazov et al., Measurement of the electron charge asymmetry in $\boldsymbol{p} \overline{\boldsymbol{p}} \rightarrow \boldsymbol{W}+\boldsymbol{X} \rightarrow \boldsymbol{e} \boldsymbol{\nu}+\boldsymbol{X}$ decays in $\boldsymbol{p} \overline{\boldsymbol{p}}$ collisions at $\sqrt{\boldsymbol{s}}=\mathbf{1 . 9 6}$ TeV, Phys. Rev. D91 (2015), no. 3 032007, arXiv:1412.2862]. [Erratum: Phys. Rev.D91,no.7,079901(2015)].

[47] H1 , ZEUS Collaboration, H. Abramowicz et al., Combination and QCD Analysis of Charm Production Cross Section Measurements in Deep-Inelastic ep Scattering at HERA, Eur.Phys.J. C73 (2013) 2311, arXiv:1211.1182.

[48] A. D. Martin, A. J. Mathijseen, W. J. Stirling, R. S. Thorne, B. J. A. Watt, and G. Watt, Extended Parameterisations for MSTW PDFs and their effect on Lepton Charge Asymmetry from $W$ Decays, arXiv:1211.1215.

[49] R. Thorne, Effect of changes of variable flavor number scheme on parton distribution functions and predicted cross sections, Phys.Rev. D86 (2012) 074017, arXiv:1201.6180.

[50] CMS Collaboration, S. Chatrchyan et al., Measurement of the muon charge asymmetry in inclusive $p p$ to WX production at $\sqrt{s}=7 \mathrm{TeV}$ and an improved determination of light parton distribution functions, Phys.Rev. D90 (2014) 032004, arXiv:1312.6283.

[51] L. A. Harland-Lang, A. D. Martin, P. Motylinski, and R. S. Thorne, Uncertainties on $\alpha_{S}$ in the MMHT2014 global PDF analysis and implications for SM predictions, arXiv:1506.05682.

[52] S. Carrazza and J. Pires, Perturbative QCD description of jet data from LHC Run-I and Tevatron Run-II, JHEP 10 (2014) 145, arXiv:1407.7031.

[53] D. de Florian, P. Hinderer, A. Mukherjee, F. Ringer, and W. Vogelsang, Approximate next-to-next-to-leading order corrections to hadronic jet production, Phys.Rev.Lett. 112 (2014) 082001, arXiv:1310.7192. 
[54] A. Gehrmann-De Ridder, T. Gehrmann, E. Glover, and J. Pires, Second order QCD corrections to jet production at hadron colliders: the all-gluon contribution, Phys.Rev.Lett. 110 (2013) 162003, arXiv:1301.7310.

[55] R. Gauld, J. Rojo, L. Rottoli, and J. Talbert, Charm production in the forward region: constraints on the small-x gluon and backgrounds for neutrino astronomy, arXiv:1506.08025.

[56] J. Rojo et al., "Chapter 22 in: J. R. Andersen et al., "The SM and NLO multileg working group: Summary report"." arXiv:1003.1241, 2010.

[57] J. Butterworth et al., Les Houches 2013: Physics at TeV Colliders: Standard Model Working Group Report, arXiv:1405.1067.

[58] S. Alekhin and S. Moch, Heavy-quark deep-inelastic scattering with a running mass, Phys. Lett. B699 (2011) 345-353, arXiv:1011.5790.

[59] S. Alekhin, J. Blümlein, L. Caminadac, K. Lipka, K. Lohwasser, S. Moch, R. Petti, and R. Placakyte, Determination of Strange Sea Quark Distributions from Fixed-target and Collider Data, Phys. Rev. D91 (2015), no. 9 094002, arXiv:1404.6469].

[60] S. Alekhin, J. Blümlein, S. Moch, and R. Placakyte, Iso-spin asymmetry of quark distributions and implications for single top-quark production at the $\mathrm{LHC}$, arXiv:1508.07923.

[61] S. Dulat et al., Intrinsic Charm Parton Distribution Functions from CTEQ-TEA Global Analysis, Phys.Rev. D89 (2014), no. 7 073004, arXiv:1309.0025.

[62] P. Jimenez-Delgado, T. Hobbs, J. Londergan, and W. Melnitchouk, New limits on intrinsic charm in the nucleon from global analysis of parton distributions, Phys.Rev.Lett. 114 (2015), no. 8 082002, arXiv:1408.1708].

[63] R. D. Ball, V. Bertone, M. Bonvini, S. Forte, P. G. Merrild, J. Rojo, and L. Rottoli, Intrinsic charm in a matched general-mass scheme, arXiv:1510.00009.

[64] S. Alekhin, J. Blümlein, K. Daum, K. Lipka, and S. Moch, Precise charm-quark mass from deep-inelastic scattering, Phys.Lett. B720 (2013) 172, arXiv:1212.2355].

[65] J. Gao, M. Guzzi, and P. M. Nadolsky, Charm quark mass dependence in a global QCD analysis, Eur.Phys.J. C73 (2013) 2541, [arXiv:1304.3494].

[66] A. D. Martin, R. G. Roberts, W. J. Stirling, and R. S. Thorne, Parton distributions incorporating QED contributions, Eur. Phys. J. C39 (2005) 155, hep-ph/0411040.

[67] A. Martin and M. Ryskin, The photon PDF of the proton, Eur.Phys.J. C74 (2014) 3040, arXiv:1406.2118.

[68] NNPDF Collaboration, R. D. Ball et al., Parton distributions with QED corrections, Nucl.Phys. B877 (2013) 290-320, arXiv:1308.0598.

[69] C. Schmidt, J. Pumplin, D. Stump, and C. P. Yuan, CT14QED PDFs from Isolated Photon Production in Deep Inelastic Scattering, arXiv:1509.02905. 
[70] S. Alekhin et al., HERAFitter, Eur. Phys. J. C75 (2015), no. 7 304, arXiv:1410.4412].

[71] HERAFitter developers' Team Collaboration, P. Belov et al., Parton distribution functions at LO, NLO and NNLO with correlated uncertainties between orders, Eur. Phys. J. C74 (2014), no. 10 3039, arXiv:1404.4234.

[72] HERAFitter developers' Team Collaboration, S. Camarda et al., QCD analysis of $W$ - and Z-boson production at Tevatron, arXiv:1503.05221.

[73] ATLAS Collaboration, G. Aad et al., Determination of the strange quark density of the proton from ATLAS measurements of the $W, Z$ cross sections, Phys.Rev.Lett. (2012) arXiv:1203.4051.

[74] CMS Collaboration, S. Chatrchyan et al., Measurements of differential jet cross sections in proton-proton collisions at $\sqrt{s}=7$ TeV with the CMS detector, Phys.Rev. D87 (2013) 112002, arXiv:1212.6660.

[75] CMS Collaboration, V. Khachatryan et al., Constraints on parton distribution functions and extraction of the strong coupling constant from the inclusive jet cross section in pp collisions at $\sqrt{s}=7$ TeV, Eur. Phys. J. C75 (2015), no. 6 288, arXiv:1410.6765.

[76] PROSA Collaboration, O. Zenaiev, A. Geiser, K. Lipka, J. Blümlein, A. Cooper-Sarkar, M.-V. Garzelli, M. Guzzi, O. Kuprash, S.-O. Moch, P. Nadolsky, R. Placakyte, K. Rabbertz, I. Schienbein, and P. Starovoitov, Impact of heavy-flavour production cross sections measured by the LHCb experiment on parton distribution functions at low x, Eur. Phys. J. C 75 (2015), no. 8 396, arXiv:1503.04581.

[77] LHeC Study Group Collaboration, J. Abelleira Fernandez et al., A Large Hadron Electron Collider at CERN: Report on the Physics and Design Concepts for Machine and Detector, J.Phys. G39 (2012) 075001, arXiv:1206.2913.

[78] G. Watt, Parton distribution function dependence of benchmark Standard Model total cross sections at the 7 TeV LHC, JHEP 1109 (2011) 069, arXiv:1106.5788.

[79] S. Alekhin, J. Blümlein, P. Jimenez-Delgado, S. Moch, and E. Reya, NNLO Benchmarks for Gauge and Higgs Boson Production at TeV Hadron Colliders, Phys. Lett. B697 (2011) 127-135, arXiv:1011.6259.

[80] R. S. Thorne and G. Watt, PDF dependence of Higgs cross sections at the Tevatron and LHC: Response to recent criticism, JHEP 1108 (2011) 100, arXiv:1106.5789.

[81] R. D. Ball, V. Bertone, L. Del Debbio, S. Forte, A. Guffanti, et al., Precision NNLO determination of $\alpha_{s}\left(M_{Z}\right)$ using an unbiased global parton set, Phys.Lett. B707 (2012) 66-71, arXiv:1110.2483.

[82] S. Alekhin, J. Blümlein, and S. Moch, Higher order constraints on the Higgs production rate from fixed-target DIS data, Eur. Phys. J. C71 (2011) 1723, arXiv:1101.5261]. 
[83] NNPDF Collaboration, R. D. Ball, V. Bertone, F. Cerutti, L. Del Debbio, S. Forte, A. Guffanti, J. I. Latorre, J. Rojo, and M. Ubiali, On the Impact of NMC Data on NLO and NNLO Parton Distributions and Higgs Production at the Tevatron and the LHC, Phys. Lett. B704 (2011) 36-42, arXiv:1102.3182.

[84] The NNPDF Collaboration, R. D. Ball et al., Theoretical issues in PDF determination and associated uncertainties, Phys.Lett. B723 (2013) 330, arXiv:1303.1189].

[85] R. Thorne, The effect on PDFs and $\alpha_{S}\left(M_{Z}^{2}\right)$ due to changes in flavour scheme and higher twist contributions, Eur.Phys.J. C74 (2014), no. 7 2958, arXiv:1402.3536].

[86] A. M. Cooper-Sarkar, Including heavy flavour production in PDF fits, arXiv:0709.0191.

[87] S. D. Ellis, Z. Kunszt, and D. E. Soper, Two jet production in hadron collisions at order alpha-s**3 in QCD, Phys.Rev.Lett. 69 (1992) 1496-1499.

[88] Z. Nagy, Three jet cross-sections in hadron hadron collisions at next-to-leading order, Phys.Rev.Lett. 88 (2002) 122003, hep-ph/0110315.

[89] Z. Nagy, Next-to-leading order calculation of three-jet observables in hadron hadron collision, Phys. Rev. D68 (2003) 094002, hep-ph/0307268.

[90] T. Carli et al., A posteriori inclusion of parton density functions in NLO QCD final-state calculations at hadron colliders: The APPLGRID Project, Eur.Phys.J. C66 (2010) 503, arXiv:0911.2985.

[91] fastNLO Collaboration, M. Wobisch, D. Britzger, T. Kluge, K. Rabbertz, and F. Stober, Theory-Data Comparisons for Jet Measurements in Hadron-Induced Processes, arXiv:1109.1310.

[92] T. Kluge, K. Rabbertz, and M. Wobisch, fastNLO: Fast $p Q C D$ calculations for PDF fits, in 14th International Workshop on Deep Inelastic Scattering (DIS 2006), (Tsukuba, Japan, April 20-24), p. 483, 2006. hep-ph/0609285

[93] D. Britzger, K. Rabbertz, F. Stober, and M. Wobisch, New features in version 2 of the fastNLO project, in 20th International Workshop on Deep-Inelastic Scattering and Related Subjects (DIS 2012), (Bonn, Germany, March 26-30), p. 217, 2012. arXiv:1208.3641.

[94] J. Gao, Z. Liang, D. E. Soper, H.-L. Lai, P. M. Nadolsky, and C. P. Yuan, MEKS: $a$ program for computation of inclusive jet cross sections at hadron colliders, Comput. Phys. Commun. 184 (2013) 1626-1642, arXiv:1207.0513.

[95] J. Gao, Z. Liang, and P. Nadolsky, "A NLO benchmark comparison for inclusive jet production at hadron colliders." Preprint SMU-HEP-12-02 (2012), in Proceedings of the 2011 Workshop "Phys ics at TeV Colliders", Les Houches, France, 2011; arXiv:1203.6803 [hep-ph], pages 77-88. 
[96] R. S. Thorne, L. A. Harland-Lang, A. D. Martin, and P. Motylinski, The Effect of Final HERA inclusive Cross Section Data MMHT2014 PDFs, in Proceedings, 2015 European Physical Society Conference on High Energy Physics (EPS-HEP 2015), 2015. arXiv:1508.06621.

[97] J. Rojo, Progress in the NNPDF global analysis and the impact of the legacy HERA combination, in Proceedings, 2015 European Physical Society Conference on High Energy Physics (EPS-HEP 2015), 2015. arXiv:1508.07731.

[98] J. Pumplin et al., New generation of parton distributions with uncertainties from global QCD analysis, JHEP 07 (2002) 012, hep-ph/0201195].

[99] J. Pumplin et al., Uncertainties of predictions from parton distribution functions. 2. The Hessian method, Phys. Rev. D65 (2001) 014013, hep-ph/0101032.

[100] J. C. Collins and J. Pumplin, Tests of goodness of fit to multiple data sets, hep-ph/0105207.

[101] G. Watt and R. S. Thorne, Study of Monte Carlo approach to experimental uncertainty propagation with MSTW 2008 PDFs, JHEP 1208 (2012) 052, arXiv:1205.4024.

[102] S. Carrazza, A. Ferrara, D. Palazzo, and J. Rojo, APFEL Web: a web-based application for the graphical visualization of parton distribution functions, J.Phys. G42 (2015) 057001, arXiv:1410.5456.

[103] V. Bertone, S. Carrazza, and J. Rojo, APFEL: A PDF Evolution Library with QED corrections, Comput.Phys.Commun. 185 (2014) 1647, arXiv:1310.1394.

[104] J. M. Campbell, J. W. Huston, and W. J. Stirling, Hard interactions of quarks and gluons: A primer for LHC physics, Rept. Prog. Phys. 70 (2007) 89, hep-ph/0611148.

[105] G. P. Salam and J. Rojo, A Higher Order Perturbative Parton Evolution Toolkit (HOPPET), Comput. Phys. Commun. 180 (2009) 120-156, arXiv:0804.3755.

[106] M. Botje, QCDNUM: Fast QCD Evolution and Convolution, Comput.Phys.Commun. 182 (2011) 490-532, $\operatorname{arXiv:1005.1481.}$.

[107] Particle Data Group Collaboration, K. Olive et al., Review of Particle Physics, Chin.Phys. C38 (2014) 090001.

[108] The NNPDF Collaboration, L. Del Debbio, S. Forte, J. I. Latorre, A. Piccione, and J. Rojo, Unbiased determination of the proton structure function f2(p) with estimation, JHEP 03 (2005) 080, hep-ph/0501067].

[109] The NNPDF Collaboration, L. Del Debbio, S. Forte, J. I. Latorre, A. Piccione, and J. Rojo, Neural network determination of parton distributions: The nonsinglet case, JHEP 03 (2007) 039, hep-ph/0701127.

[110] The NNPDF Collaboration, R. D. Ball et al., A determination of parton distributions with faithful uncertainty estimation, Nucl. Phys. B809 (2009) 1-63, arXiv:0808.1231. 
[111] J. Gao and P. Nadolsky, A meta-analysis of parton distribution functions, JHEP 1407 (2014) 035, arXiv:1401.0013.

[112] S. Carrazza, S. Forte, Z. Kassabov, J. I. Latorre, and J. Rojo, An Unbiased Hessian Representation for Monte Carlo PDFs, Eur. Phys. J. C75 (2015), no. 8 369, arXiv:1505.06736.

[113] S. Carrazza, J. I. Latorre, J. Rojo, and G. Watt, A compression algorithm for the combination of PDF sets, Eur. Phys. J. C75 (2015), no. 10 474, arXiv:1504.06469.

[114] G. Watt, talk presented at PDF4LHC meeting, 17th April 2013, CERN, Geneva. http://indico.cern.ch/event/244768/contribution/5.

[115] A. Buckley, J. Ferrando, S. Lloyd, K. Nordstrm, B. Page, et al., LHAPDF6: parton density access in the LHC precision era, Eur.Phys.J. C75 (2015) 132, arXiv:1412.7420.

[116] CMS Collaboration, S. Chatrchyan et al., Measurement of associated $W+$ charm production in pp collisions at $\sqrt{s}=7$ TeV, JHEP 02 (2014) 013, arXiv:1310.1138.

[117] V. Bertone, R. Frederix, S. Frixione, J. Rojo, and M. Sutton, aMCfast: automation of fast NLO computations for PDF fits, JHEP 1408 (2014) 166, arXiv:1406.7693.

[118] J. Alwall, R. Frederix, S. Frixione, V. Hirschi, F. Maltoni, et al., The automated computation of tree-level and next-to-leading order differential cross sections, and their matching to parton shower simulations, JHEP 1407 (2014) 079, arXiv:1405.0301.

[119] LHCb Collaboration, R. Aaij et al., Measurement of the cross-section for $Z \rightarrow e^{+} e^{-}$ production in pp collisions at $\sqrt{s}=7$ TeV, JHEP 1302 (2013) 106, arXiv:1212.4620].

[120] ATLAS Collaboration, G. Aad et al., Measurement of the high-mass Drell-Yan differential cross-section in pp collisions at $\sqrt{s}=7$ TeV with the ATLAS detector, Phys.Lett. B725 (2013) 223, arXiv:1305.4192.

[121] ATLAS Collaboration, G. Aad et al., Measurement of the Transverse Momentum Distribution of $W$ Bosons in pp Collisions at $\sqrt{s}=7$ TeV with the ATLAS Detector, Phys.Rev. D85 (2012) 012005, arXiv:1108.6308.

[122] CMS Collaboration, S. Chatrchyan et al., Measurement of the differential and double-differential Drell-Yan cross sections in proton-proton collisions at $\sqrt{s}=7 \mathrm{TeV}$, JHEP 1312 (2013) 030, arXiv:1310.7291.

[123] ATLAS Collaboration, G. Aad et al., Measurement of the inclusive jet cross section in pp collisions at $\sqrt{s}=2.76$ TeV and comparison to the inclusive jet cross section at $\sqrt{s}=7$ TeV using the ATLAS detector, Eur.Phys.J. C73 (2013) 2509, arXiv:1304.4739.

[124] CMS Collaboration, V. Khachatryan et al., Measurement of the inclusive 3-jet production differential cross section in protonproton collisions at $7 \mathrm{TeV}$ and determination of the strong coupling constant in the TeV range, Eur. Phys. J. C75 (2015) 186, arXiv:1412.1633. 
[125] G. Bozzi, J. Rojo, and A. Vicini, The Impact of PDF uncertainties on the measurement of the $W$ boson mass at the Tevatron and the LHC, Phys.Rev. D83 (2011) 113008, arXiv:1104.2056.

[126] CMS Collaboration, S. Chatrchyan et al., Determination of the top-quark pole mass and strong coupling constant from the $t$ t-bar production cross section in pp collisions at $\sqrt{s}=7$ TeV, Phys.Lett. B728 (2014) 496, arXiv:1307.1907.

[127] W. Beenakker, C. Borschensky, M. Krmer, A. Kulesza, E. Laenen, S. Marzani, and J. Rojo, NLO+NLL squark and gluino production cross-sections with threshold-improved parton distributions, arXiv:1510.00375.

[128] S. Aoki et al., Review of lattice results concerning low-energy particle physics, Eur. Phys. J. C74 (2014) 2890, arXiv:1310.8555.

[129] S. Alioli, P. Nason, C. Oleari, and E. Re, A general framework for implementing NLO calculations in shower Monte Carlo programs: the POWHEG BOX, JHEP 1006 (2010) 043, arXiv:1002.2581.

[130] T. Gleisberg et al., Event generation with SHERPA 1.1, JHEP 02 (2009) 007, arXiv:0811.4622.

[131] R. Gavin, Y. Li, F. Petriello, and S. Quackenbush, W Physics at the LHC with FEWZ 2.1, Comput.Phys.Commun. 184 (2013) 208-214, arXiv:1201.5896.

[132] C. Balazs and C. P. Yuan, Soft gluon effects on lepton pairs at hadron colliders, Phys. Rev. D56 (1997) 5558-5583, hep-ph/9704258.

[133] F. Landry, R. Brock, P. M. Nadolsky, and C. P. Yuan, Tevatron Run-1 Z boson data and Collins-Soper-Sterman resummation formalism, Phys. Rev. D67 (2003) 073016, hep-ph/0212159.

[134] F. Maltoni, G. Ridolfi, and M. Ubiali, b-initiated processes at the LHC: a reappraisal, JHEP 07 (2012) 022, arXiv:1203.6393. [Erratum: JHEP04,095(2013)].

[135] L. A. Harland-Lang, A. D. Martin, P. Motylinski, and R. S. Thorne, Charm and beauty quark masses in the MMHT2014 global PDF analysis, arXiv:1510.02332.

[136] V. Bertone, S. Carrazza, and J. Rojo, Doped Parton Distributions, in 27th Rencontres de Blois on Particle Physics and Cosmology Blois, France, May 31-June 5, 2015, 2015. arXiv:1509.04022.

[137] K. G. Chetyrkin, J. H. Kuhn, and C. Sturm, QCD decoupling at four loops, Nucl. Phys. B744 (2006) 121-135, hep-ph/0512060.

[138] M. Bonvini, S. Marzani, J. Rojo, L. Rottoli, M. Ubiali, R. D. Ball, V. Bertone, S. Carrazza, and N. P. Hartland, Parton distributions with threshold resummation, JHEP 09 (2015) 191, arXiv:1507.01006.

[139] J. A. M. Vermaseren, A. Vogt, and S. Moch, The third-order QCD corrections to deep-inelastic scattering by photon exchange, Nucl. Phys. B724 (2005) 3, hep-ph/0504242. 
[140] S. Moch, J. A. M. Vermaseren, and A. Vogt, The longitudinal structure function at the third order, Phys. Lett. B606 (2005) 123, hep-ph/0411112. 